\title{
Turbulence structure and interaction with steep breaking waves
}

\author{
DJAMEL LAKEHAL ${ }^{1} \dagger$ AND PETAR LIOVIC 2 \\ ${ }^{1}$ ASCOMP GmbH Zürich, Technoparkstrasse 1, H22, Zurich 8005, Switzerland \\ ${ }^{2}$ CSIRO Materials Science and Engineering, Graham Road, Highett VIC 3190, Australia \\ (Received 7 December 2009; revised 24 December 2010; accepted 29 December 2010; \\ first published online 4 April 2011)
}

Large-eddy and interface simulation using an interface tracking-based multi-fluid flow solver is conducted to investigate the breaking of steep water waves on a beach of constant bed slope. The present investigation focuses mainly on the 'weak plunger' breaking wave type and provides a detailed analysis of the two-way interaction between the mean fluid flow and the sub-modal motions, encompassing wave dynamics and turbulence. The flow is analysed from two points of views: mean to sub-modal exchange, and wave to turbulence interaction within the sub-modal range. Wave growth and propagation are due to energy transfer from the mean flow to the waves, and transport of mean momentum by these waves. The vigorous downwellingupwelling patterns developing at the head and tail of each breaker are shown to generate both negative- and positive-signed energy exchange contributions in the thin sublayer underneath the water surface. The details of these exchange mechanisms are thoroughly discussed in this paper, together with the interplay between threedimensional small-scale breaking associated with turbulence and the dominant twodimensional wave motion. A conditional zonal analysis is proposed for the first time to understand the transient mechanisms of turbulent kinetic energy production, decay, diffusion and transport and their dependence and/or impact on surface wrinkling over the entire breaking process. The simulations provide a thorough picture of airliquid coherent structures that develop over the breaking process, and link them to the transient mechanisms responsible for their local incidence.

Key words: air/sea interactions, coastal engineering, wave breaking

\section{Introduction}

The generation of alongshore currents by surface-wave breaking is one of the mechanisms best translating two-way, nonlinear interactions between the mean fluid flow and the waves. To set the context of the paper for conceptual purposes, the mean flow (obtained through an averaging procedure) is hereafter distinguished from the wave motions and turbulence on the basis of both frequency and spatial scales. Waves have higher frequency and small spatial scales than the mean flow, but still smaller frequency and larger spatial scales than turbulence. A detailed separation of scales by means of computational fluid dynamics is very elusive, precisely because of the variety of physical processes proper to both the mean flow, the waves and subscale turbulence. To reduce the scope of the analysis, 
we also define modal wave motions or model range as the portion of spectrum encompassing wave and subscale turbulence. Energy exchange mechanisms could thus be restricted to analyse mean to modal range transfer, or, when possible, from wave to subscale turbulence range within the modal range. Furthermore, as the interest of the present investigation is on the interactions between the mean and modal range in the interfacial region separating air and water phases, we define the sea upper- and subsurface layers as the air- and water-sided interfacial flow regions, respectively, where flow vertical gradients can be markedly sensitive to surface displacements.

The two-way interaction mechanism between mean flow and modal ranges (wave plus turbulence) operates in a strongly nonlinear form, because both the waves and the mean fluid flow often involve very different spatial and temporal scales, responsible for different physical processes (i.e. propagation speed and length versus turbulent cascades). The fluid flow and wave kinematics can be very sensitive to small perturbations imposed on the sea upper surface (e.g. imposed shear and pressure fluctuations) and the sea sub-surface layers (e.g. upwellings and downdrafts). The nonlinearity of the interaction process manifests itself primarily in the momentum transfer between wind and ocean currents via wave breaking. Large-scale wave breaking leading to whitecapping and surface-foam formation is the most visible sign of nonlinearity in the process. The less visible, small-scale breaking is rather controlled by surface instabilities of the smaller-amplitude waves superposed on the large-scale waves. Combined large-to-small scale wave breaking is known to disrupt the ocean's cool skin (Moum \& Smyth 2002).

While the large-scale motion of waves including wave-breaking events is now relatively well understood, the same cannot be said of the interaction between turbulence and small-scale surface deformations subsequent to wave breaking. The measurements of Thorpe et al. (2003) have shown that wave breaking dominates the near-surface turbulence and that shear-induced eddies (along with Langmuir circulation) are important processes of a few times the wave height below the surface. Breaking waves are a source of intense turbulence that manifests itself in various flow regimes (Peregrine 1983; Duncan 2001). As a source of enhanced turbulent kinetic energy (TKE) and dissipation of wave energy, wave breaking plays a key role in upper-ocean processes. Vertical momentum and scalar transport, as well as sea-spray dispersion and deposition in the near-surface zone, are known to be controlled by turbulence (Lin \& Hwung 1992; Thorpe 1995; Gemmrich \& Farmer 1999). For waves of small wave-slope subject to weak breaking of the crest, the upper-surface layer conserves the form of stratified flow in which the fluids remain immiscible. In this case, the vertical surface-to-atmosphere scalar transfer (evaporation) and flux distortion are mainly controlled by the convective flow in the air. The extreme scenario featuring vigorous wave plunging leads to the bursting of air bubbles into sea spray droplets. The dispersion in the airflow of these droplets is (to a large extent) controlled by the air-side turbulence, while their deposition is controlled by the pressure and shear-induced surface deformations (Botto et al. 2005). Furthermore, because it increases surface area, wave breaking is responsible for enhancing the transfer of heat and mass resulting from sea-spray evaporation (Melville 1996).

In terms of scale separation, wave breaking is associated with a multitude of flow length and time scales, ranging from centimetre-scale capillary waves, through wind waves (tens of metres) to swell (hundreds of metres) (Moum \& Smyth 2002). In rare low-wind conditions, winds produce a surface stress and a sheared 
current profile, resulting in a wall-layer scaling of turbulent fluxes in the oceanic surface layer similar to the atmospheric surface layer. This similarity has been demonstrated by both theory and experimental observations (Thorpe 1993; Moum \& Smyth 2002), and recently by direct numerical simulation (DNS) (Fulgosi et al. 2003). In real ocean wave conditions however, sea and atmospheric surface layers behave quite differently, because the ocean's upper boundary is free to support waves of various scales. Therefore, one might expect differences in the mechanisms of energy transfer and dissipation. Turbulence observations in the oceanic surface layer under higher winds and more complicated wave states have indeed shown that turbulence dissipation rates greatly exceed those predicted by wall-layer scaling (Thorpe 1993).

The multiple possibilities for scaling of turbulent fluxes suggest that a very subtle two-way energy cascade exists in the oceanic surface layer. At the sea upper-surface layer, as water waves propagate, kinetic energy is transferred from the primary incident wave to higher-frequency waves through wave-wave interactions. This mechanism is comparable but not necessarily similar to the TKE cascade under high-Reynoldsnumber flow conditions, where energy is transferred from the integral to the dissipative scales. In real ocean conditions, it happens that both energy cascade mechanisms act in concert. The manner and reason these mechanisms act in concert is currently not well understood because neither the underlying wave-breaking process nor its interaction with turbulence are well described. This problem may be better termed as a 'multi-fluid scale separation', manifesting itself essentially in the high-frequency flow regime. In this scenario, interfacial scales (including air bubbles and water droplets) generated by surface wave fragmentation interact essentially with turbulent flow motions of equivalent length scales. How that happens is an interesting question that the present paper intends to address. Other mechanisms associated with production, dissipation and transport of turbulence, and short-wave breaking accompanying large-scale surface breaking also aim to be clarified.

In this paper, coupled turbulence dynamics and wave-breaking phenomena are numerically investigated by incorporating the physics pertaining to the air and liquid flow and entrainment induced by wave motion and breaking. Understandably, the complexity of the flow requires the use of higher-fidelity representations of surface topology than free-surface flow codes and two-fluid formulations, and more advanced physical models for turbulence than statistically time-averaged approaches. Highfidelity solutions to wave-breaking - with explicit focus on turbulence-interface interactions - require the use of interface tracking methods for surface topology changes and large-eddy simulation (LES) for turbulence. This combination is now known as LEIS - short for large-eddy and interface simulation - where only supergrid turbulence and interfacial scales are fully resolved. The choice of a weak plunger breaking scenario for highlighting the utility of LEIS is justified since this flow prototype features mild wave breaking under a limited shear Reynolds number $(\geqslant 400)$. In the main flow problem considered here, the breaking of steep waves is gravity-driven, forced by the upward incline of a beach at constant bed slope; the increased height of the wave indeed results in the wave perceiving bottom further offshore.

\section{Wave breaking and turbulence}

A comprehensive review of the wave-breaking process based on various earlier experimental findings and observations was carried out by Christensen, Walstra \& 
Emerat (2002). Breaking seems to occur when the horizontal velocity of water at the wave crest exceeds the wave speed; the same is true for the velocity at the tip of overturning waves (Chang \& Liu 1999). At the impact of the overturning wave, random vertical vortices may appear, favouring the organization of 'obliquely descending eddies' (Nadaoka, Hino \& Koyano 1989). Turbulent structures are created at the top of breaking waves, such that production mainly occurs in this upper region. These flow structures then break down and interact with the undertow flow in the middle region below trough level, and there is further interaction between the middle region and the boundary layer of the seabed. Watanabe, Saeki \& Hosking (2005) numerically studied the three-dimensional (3D) vortex structures under breaking waves to elucidate the turbulence phenomenology associated with an overturning jet projecting from the crest in a breaking wave that rebounds from the water surface ahead. They reported the formation of a primary spanwise vortex, resulting in spanwise undulations of the vorticity, which then get amplified on a braid leading to a vortex loop with counter-rotating vorticity. The explanation of the subsequent vorticity dynamics under the action of the overturning jet traces back the roots of the obliquely descending eddies described by other authors hitherto.

Wave breaking is considered to be the most visible process in which waves dissipate energy, with the organized wave energy of rollers being first converted into TKE in the form of organized vortices, and then dissipated into small-scale turbulent motion. It is unlikely that this dissipation occurs in an isotropic way as postulated by Roelvink \& Stive (1989). Furthermore, spilling and plunging breakers differ with regard to prevailing turbulent transport processes. In the spilling case, turbulence preserves some self-similar behaviour during a wave cycle. In the plunging scenario, it first exhibits turbulent plume characteristics at the wave break and very marginal activities away from the plunging area. More pointedly, Ting \& Kirby (1994) concluded that TKE is transported seawards under the spilling breaker and is dissipated slowly, while it is transported landward under the plunging breaker and dissipated within one cycle. Ting (2001) also advocated the notion of TKE in breaking waves being transported to the bottom by convection and turbulent diffusion, and transported upwards by the undertow.

Lin et al. (2008) have recently conducted DNS of wind-wave generation processes at low wind speed and mild surface wind stress and associated frictional velocities of the air and the water. Their study revealed that the wave growth consists of linear and exponential growth stages, as suggested by theoretical and experimental studies. They have also examined the effects of waves on turbulence statistics above and below the interface and found that the wave effect on the turbulent velocity variances is different in air and water. While in the air the vertical distributions of the velocity variances are in close agreement with wall-bounded shear turbulent flows, the velocity variances in the water are strongly affected by waves.

\section{Numerical simulation of free-surface waves}

The numerical prediction of wave breaking in coastal engineering research has traditionally been based on free-surface models (using single-fluid flow solvers) imposing zero-pressure boundary conditions (e.g. Christensen \& Deigaard 2001; Zhao, Armfield \& Tanimoto 2004). Some of the wave breaking features investigated by such models include surface rollers at the front of spilling breakers, the dynamics of water tongues while jetting forward to impact on the surface in front of the 
crest, flow patterns under breaking waves, and the generation and spreading of turbulence by wave deformations. Water-flow simulations, on their own, provide no insight into the airflow above the free surface; only half of the interfaceturbulence interactions' story is therefore targeted. Wind effects on wave motions and breaking, and the subsequent scalar absorption by waters, cannot be handled naturally without detailed coupled air-water flow solutions. Imposing a free-surface pressure boundary condition may not be adequate; Jenkins (2002) noted significant contradictions in the understanding of wind waves when treated by these simplified approaches. For steep-wave propagation and breaking, form drag is not distributed uniformly across the surface as may be implied by a zero-pressure boundary condition. In wave plunging, events such as the propagation of the water tongue ahead of the wave crest can entrain a substantial amount of air (Lamarre \& Melville 1991). The use of free-surface models that ignore air entrainment may thus lead to significant discrepancies in the water flow solution and necessarily in the associated interfacial scalar transfer mechanisms. We will show here that the steady-state conditions are mainly altered by this precise wave-roll-induced air entrainment.

DNS studies dealing with non-stationary water waves are rare, with the work of Sullivan McWilliams \& Moeng (2000) being a notable exception. High-amplitude waves $(a k=0.1)$ were found in that contribution to significantly influence the characteristics of the mean flow and turbulence. In comparison to stationary waves, slow-moving waves were found to increase the form drag, whereas fast-moving waves decrease it. On the basis of the DNS tool Sullivan et al. (2000) employed for the flow over moving water waves, Sullivan, McWilliams \& Melville (2004) simulated the boundary layer driven by wave breaking. To represent the stress and energy transfer from breaking waves to boundary layer currents and turbulence, a 3D random forcing was added to the resolved momentum equations and a random work density to the subgrid-scale (SGS) turbulent kinetic energy equation. Although the time and location of breaking events were randomly distributed in the model, breaking waves were considered as coherently structured events resembling those observed in flat-wall boundary layers. One aspect of breaking not included in their model was air entrainment, which they noted to be potentially important for large active breakers.

Alternatively, there have been relatively few DNS-based studies of turbulence in problems involving two-phase flows, if one excludes earlier research focusing on freesurface turbulence with and without wind shear (Lam \& Banerjee 1992; Komori et al. 1993b; Lombardi, DeAngelis \& Banerjee 1996). Because of its relative simplicity, stratified gas-liquid flow has been the configuration best suited to investigating the underlying physics of turbulence at the interface. The DNS investigation of Lombardi et al. (1996) concentrated on a flat interface configuration, where gas and liquid were coupled through continuity of velocity and stress jump conditions at the interface. The authors found that the lighter phase might look at the interface like a solid surface. Fulgosi et al. (2003) extended the research of Lombardi et al. (1996) to non-flat, sheared interfaces by considering stratified flow with a freely deformable interface in the gravity-capillary wave regime. With regard to wave breaking, multiple contributions have been made using LES (Christensen 2006; Lubin et al. 2006). For example, Lubin et al. (2006) and Christensen (2006) used the volume-of-fluid (VOF) method to track the free surface, albeit no specific treatment of turbulence at the interface was reported in either contribution. However, while Lubin et al. (2006) used a sharp interface reconstruction scheme also featuring surface tension, Christensen 
(2006) did not feature such high-fidelity interface tracking and imposed a zeropressure boundary condition at the interface. Other numerical simulations (Lin \& Liu 1998) were presented, though assuming that two-dimensional (2D) turbulence is statistically predominant to justify the resort to a statistical time-averaged approach, i.e. Reynolds-averaged Navier-Stokes (RANS) turbulence modelling. Watanabe \& Saeki (1999) investigated the vorticity evolution and the emergence of the spanwise velocity component during wave breaking in three dimensions. They resorted to LES to track turbulence, with an SGS viscosity model based on the renormalization group theory. Their work was later on extended by Watanabe et al. (2005) to investigate the vorticity and strain fields for both plunging and spilling breakers.

\section{Mathematical formulation}

\subsection{The LEIS concept for interfacial flows}

Interfacial flows refer to two-phase flow problems involving two immiscible fluids separated by a deformable sharp interface. Under turbulent conditions such as those that prevail in wave-breaking scenarios, the flow exhibits scale variations within each phase, the spectrum of which obeys the 'turbulence dissipation cascade'. These can be termed as phase-specific turbulent scales (TS). On the other hand, surface deformations associated with wave breaking give birth to a broad range of interfacial scales (IS), ranging from sea sprays to large-wave curvature. Understandably, solving directly all the flow scales (DNS) is rather elusive. Instead, one may resort to resolving only the supergrid scales (or large eddies and large interfaces), leaving the rest (e.g. phase-specific Kolmogorov scales and sea-spray droplets) to be modelled. Any computational approach (e.g. VOF) that is capable of treating distinctively this separation of scales could be termed as LEIS. The approach should also be based on the filtered Navier-Stokes equations (Liovic \& Lakehal $2007 b$ ), reformulated though for two-phase flow using the single-fluid description. The non-resolved or SGS turbulence scales could be modelled using an eddyviscosity approach, though accounting for the presence of deformable interfaces. The representation of the subgrid interfacial scales (sea spray droplets and surface foam or whitecap) raises other non-resolved issues, including their interaction with turbulence. The material presented below intends to introduce the main ingredients of LEIS.

\subsection{The filtered one-fluid equations}

The macro-scale description of the immiscible Newtonian two-fluid flow is based on filtering the SGS flow motions $\left(f^{\prime}\right)$ out of the true flow field $(f)$ using convolution:

$$
\bar{f}(x, t) \equiv \int_{D} G\left(\boldsymbol{x}-\boldsymbol{x}^{\prime}\right) f\left(\boldsymbol{x}^{\prime}, t\right) \mathrm{d} \boldsymbol{x}^{\prime},
$$

where $G$ is a spatial filter and $\int_{D} G\left(\boldsymbol{x}-\boldsymbol{x}^{\prime}\right) \mathrm{d} \boldsymbol{x}^{\prime}=1$. In interface tracking methods, an indicator function $C(\boldsymbol{x}, t)$ is introduced to delineate points in space momentarily occupied by the gas phase $(k=G)$ or the liquid phase $(k=L)$. The simplest phase indicator function is one that sets $C(\boldsymbol{x}, t)$ equal to unity if point $\boldsymbol{x}$ is occupied by the gas phase and zero otherwise. Spatial filtering of this indicator function yields the 
volume fraction indicator function, defined by

$$
\bar{C}(x, t) \equiv \int_{D} G\left(\boldsymbol{x}-\boldsymbol{x}^{\prime}\right) C\left(\boldsymbol{x}^{\prime}, t\right) \mathrm{d} \boldsymbol{x}^{\prime},
$$

This is the discrete colour function of the VOF interface tracking method, and makes VOF an ideal foundation for consistency between the filtered and microscopic forms of the conservation laws. The derivation of the filtered onefluid equations is based on the use of the component-weighted volume-averaging (CWVA) procedure (Lakehal et al. 2002). In the context of the interface tracking-based single-fluid formalism, CWVA reduces to Favre averaging, i.e. $\widetilde{f}(\boldsymbol{x}, t)=\overline{\rho f(\boldsymbol{x}, t)} / \bar{\rho}$, where $\bar{\rho} \equiv \sum_{k} \overline{C^{k}(\boldsymbol{x}, t) \rho^{k}}$. Detailed derivation of the filtered single-fluid conservation equations is provided in Liovic \& Lakehal $(2007 a, b)$.

The filtered single-fluid equations for turbulent multi-fluid flows take the form:

$$
\begin{aligned}
\frac{\partial \bar{C}}{\partial t}+\frac{\partial\left(\bar{C} \tilde{u}_{j}\right)}{\partial x_{j}} & =0, \\
\frac{\partial \bar{\rho}}{\partial t}+\frac{\partial\left(\bar{\rho} \tilde{u}_{j}\right)}{\partial x_{j}} & =0, \\
\frac{\partial \bar{\rho} \tilde{u}_{i}}{\partial t}+\frac{\partial}{\partial x_{j}}\left(\bar{\rho} \widetilde{u}_{i} \widetilde{u}_{j}-\bar{\Pi}_{i j}+\tau_{i j}\right) & =F_{g}+F_{s}+\sum \varepsilon^{u},
\end{aligned}
$$

where $\bar{\Pi} \equiv(-\bar{p} I+\widetilde{\sigma})$ is the resolved Cauchy stress, and $F_{g}$ is the gravity force. Within the resolved Cauchy stress, a constitutive relation for Newtonian fluids is used, with viscosity $\bar{\mu}$ being computed in a $C$-weighted manner (like $\bar{\rho}$ ). The resolved surface tension force is denoted by $F_{s}=\overline{\gamma \kappa \hat{n}_{i} \delta_{s}}$, where $\gamma$ is the surface tension coefficient, $\kappa$ is the interfacial curvature, $\hat{n}_{i}$ is the unit interface normal, and $\delta_{s}$ is the surface delta function. For incompressible flows, (4.4) can be reduced to a pure continuity equation $(\nabla \cdot \widetilde{\boldsymbol{u}}=0)$. The term $\sum \varepsilon^{u}=\varepsilon_{c}^{u}+\varepsilon_{\gamma}^{u}+\varepsilon_{d}^{u}$ in the filtered momentum equation encompasses filtering-induced quantities from convection $\left(\varepsilon_{c}^{u}\right)$, surface tension $\left(\varepsilon_{\gamma}^{u}\right)$ and diffusion $\left(\varepsilon_{d}^{u}\right)$, respectively. The sum of commutation errors is contained in $\varepsilon_{c}^{u}$, while the inter-phase net force between the two fluids at the subgrid level is represented by $\varepsilon_{d}$ defined by the identity $\varepsilon_{d} \equiv \nabla(\bar{\sigma}-\widetilde{\sigma})$. This latter term could, for example, represent the drag and lift forces of a bubble of diameter smaller than the filter width. Similarly, another term

$$
\varepsilon_{\gamma}^{u}=\gamma(\overline{\kappa n}-\bar{\kappa} \bar{n}) \delta
$$

appears as a nonlinearity error concentrated at the interface, representing the nonresolved counterpart of surface tension (Liovic \& Lakehal 2007a, 2009b). Finally, in (4.5) we have introduced the local (phase-specific) SGS tensor, defined as

$$
\tau_{i j} \equiv \bar{\rho}\left(\widetilde{u_{i} u_{j}}-\widetilde{u_{i}} \widetilde{u_{j}}\right)
$$

\subsection{Subgrid scale modelling}

The $\varepsilon_{c}^{u}$ term can be neglected in the current work because of the use of uniform meshes. The other SGS terms in (4.7) have problem-specific physics and interface topology considerations associated with them, and their modelling (or neglect) should be prescribed based on physical grounds if they are indeed sufficiently documented. 


\subsubsection{Motivations in the wave-breaking flow}

According to Christensen \& Deigaard (2001), the most important breaker types are spilling breakers, plunging breakers and surging breakers. A well-known parameter for predicting breaker type given known wave propagation characteristics and near-shore seabed geometry is the surf-similarity parameter (Battjes 1975),

$$
\xi_{0}=\frac{\tan (\beta)}{\sqrt{H_{0} / L_{0}}},
$$

where $\beta$ is the bed slope, and $H_{0}$ and $L_{0}$ are the height and wavelength of the wave propagating in deep water, respectively. On the basis of $(4.8), \xi_{0} \approx 0.5$ is supposed to identify the transition from spilling waves to plunging waves.

A motivation for this work is the flow associated with the transition between spilling and plunging, and specifically associated with the 'weak plunger' flow regime (Christensen \& Deigaard 2001). An interesting observation regarding (4.8) is that, all else being equal, a steeper wave resulting from an increased value of $H_{0}$ reduces $\xi_{0}$ and hence should result in transition from plunging to spilling wave breaking. The lack of a widely accepted and unique 'weak plunger' definition (Battjes 1975) makes the regime delineation using the surf-similarity parameter particularly fuzzy and worth remedying. Taking the example of the three wave conditions investigated in Christensen \& Deigaard (2001, hereafter CD), a $50 \%$ increase in wave height results in $\xi_{0}<0.5$ across the board - from $0.30,0.44$ and 0.56 for the CD cases, to $0.24,0.36$ and 0.46 for the $50 \%$ higher waves. Superficially looking at the manner in which huge waves appear to break as strong plungers in the surf zones of Hawaii, it appears counterintuitive for increasing wave heights in the CD problem set-ups to make the waves tend to spillers. For now, persevering with the prediction from (4.8) that increasing wave height in the upscaling will make the breaking waves tend towards spillers, it is appropriate to first tailor the SGS models to the known features of spilling breakers and the deduced requirements for spilling breaker simulation.

\subsubsection{Prescribed treatments}

A novel first SGS model for unresolved surface tension (or interfacial subscales) has recently been presented by Liovic \& Lakehal (2009b). In the model, the direct estimation of $\varepsilon_{\gamma}^{u}$ in the solution algorithm is replaced with a direct evaluation of the unfiltered surface tension force $\gamma \kappa \hat{n}_{i} \delta_{s}$. This is made possible by decomposition of the curvature and interface normal components into supergrid scale and SGS components:

$$
\gamma \kappa \hat{n}_{i} \delta_{s}=\gamma\left(\bar{\kappa}+\kappa^{S G S}\right)\left(\overline{\hat{n}_{i}}+\hat{n}_{i}^{S G S}\right) \delta_{s} .
$$

Knowing that unresolved interface curvature contributions $\kappa^{S G S}$ swamp the unresolved interface normal contributions $\hat{n}_{i}^{S G S}$ simplifies the implementation of the model. The technique is based on a novel heuristics procedure for the analysis of filtered curvature estimates that are generated via two alternative routes. The first route involves discretization-based curvatures generated by applying regular interface normal and curvature discretization schemes to coarse-filtered representations of the interface. The second route involves directly filtered curvatures generated by coarsefiltering discretization-based curvature estimates using the regular-mesh (non-coarsefiltered) interface representation. The details of the model have been presented by Liovic \& Lakehal (2009b), who also provided more insight into the manner in which differences between discretization-based curvatures and directly filtered curvatures 
identify non-negligible $\kappa^{S G S}$. Application of SGS modelling techniques for unresolved interfacial scales (and, in turn, surface tension) remains a problem-dependent issue. Liovic \& Lakehal (2007b) used a priori evaluation for order-of-magnitude analysis of the unresolved surface tension term relative to the bulk phase-specific SGS stress term (4.7). For large bubble rise, the SGS stress term was found to be at least $O\left(10^{2}\right)$ larger than the unresolved surface tension. Accounting for unresolved surface tension in LEIS is perhaps necessary only when the flow features a very high concentration of small structures approaching in size the filter scale; the best example is atomization of fuel jets. Non-negligible unresolved surface tension may arise in strong plunger events, and is associated with the jet and ligament-sheet breakup that lead to the generation of sea spray drops, as well as bubble entrainment in the surf zone. The generation of foam bubbles and sea spray is, however, not a key feature of less violent wave-breaking scenarios dominated by weak plungers or spillers. As such, the current work neglects the unresolved surface tension and the inter-phase transfer term, i.e. set $\varepsilon_{\gamma}^{u}=\varepsilon_{d}^{u}=0$.

Explicit closure of the SGS stress tensor relating to turbulence in LEIS is achieved within the eddy-viscosity framework, where $\tau_{i j}$ is made proportional to the resolved strain rate $\widetilde{S}_{i j}$ :

$$
\tau_{i j}=-2 v_{t} \widetilde{S}_{i j}+\delta_{i j} \tau_{k k} / 3, \quad \text { with } \quad v_{t} \equiv f_{\mu I n t}\left(C_{s} \bar{\Delta}\right)^{2} \sqrt{2 \widetilde{S}_{i j} \widetilde{S}_{i j}},
$$

where the length scale is based on the cell size $\bar{\Delta}$, and the value of the model coefficient is set equal to $C_{S}=0.1$ in the core flow. In the presence of shear - either near the wall or in the vicinity of deformable surfaces where the viscous sublayer is well resolved eddy-viscosity models generally need to incorporate a damping function $\left(f_{\mu}\right)$ in order to accommodate the near-wall/interface limiting behaviour. Similar 'corrections' need to be introduced when eddy-viscosity models are employed for interfacial two-phase flows, where the lighter phase perceives the surface like a rigid wall (Fulgosi et al. 2003). A systematic LES study of the Fulgosi et al. (2003) flow performed by Reboux, Sagaut \& Lakehal (2006) has shown that without such modification, the Smagorinsky model alone becomes excessively dissipative, just as it tends to be for wall-bounded flows. For low-to-moderate interface deformations, the DNS database of Fulgosi et al. (2003) provided an exponential dependence of $f_{\mu I n t}$ on the distance to the interface $y_{I n t}^{+}$. For the airflow, the dependence is

$$
f_{\mu I n t}=1-\exp \left(a y_{\text {Int }}^{+}+b\left(y_{\text {Int }}^{+}\right)^{2}+c\left(y_{\text {Int }}^{+}\right)^{3}\right),
$$

where $a=-0.00013, b=-0.00036$ and $c=-1.08 \times 10^{-5}$. For the liquid side, it is

$$
f_{\mu I n t L}=1-\exp \left(f y_{\text {Int }}^{+}+g\left(y_{\text {Int }}^{+}\right)^{2}\right),
$$

where $f=-0.0014$ and $g=-0.00064$.

Analogous to the concept of a 'wall units' length scale used in near-wall turbulence modelling $\left(y^{+}\right)$, damping of eddy viscosity in SGS modelling close to sheared interfaces requires the introduction of its own non-dimensional length scale. This is denoted here by the 'interface turbulence units' length scale $y_{I n t}^{+}$. In a companion paper (Liovic \& Lakehal 2007b), the authors have introduced and validated the algorithm for extracting the ingredients necessary for estimating this length scale from the flow field - namely the air-side interfacial shear/friction velocity $U_{\tau \mid \mathrm{nt}}$, and the reconstructed distance function (RDF) from raw VOF data $\phi^{R D F}$ defined by

$$
y_{\text {Int }}^{+}=U_{I n t}^{\tau} \phi^{R D F} / \nu^{G}, \quad U_{I n t}^{\tau}=\sqrt{\tau_{\text {Int }} / \rho_{G}} .
$$


A summary of the technique is presented in Section 5 below. Note that given the dependence of $y_{\text {Int }}^{+}$on local flow conditions, the width of interface support within which $0<f_{\mu I n t}<1$ may fluctuate significantly during the simulation. The SGS model modified with (4.11) has been validated by Liovic \& Lakehal (2007b) and Reboux et al. (2006) in the context of the stratified air-water counter-current flow of Fulgosi et al. (2003).

\section{Numerical method}

\subsection{Interface tracking and the flow solver}

The MFVOF-3D flow software was developed for the simulation of transient highdensity-ratio, multi-fluid flows, with strong topology changes including fragmentation and coalescence (Liovic, Liow \& Rudman 2001). The finite-volume flow code uses a staggered-grid arrangement, and a two-step projection method for pressure-velocity coupling. The pressure Poisson equation is solved using an efficient and robust multigrid-preconditioned additive Schwarz GMRES solver. Interfaces are tracked using a VOF method updated on a twice-as-fine mesh, allowing for a rudimentary capturing of SGS curvature independent of any explicit SGS curvature modelling. A VOF-augmented momentum advection scheme ensures conservation and stability in high-density ratio, high-shear flows by ensuring transportiveness and promoting sharpness of flow momentum discontinuities at moving interfaces (Liovic et al. 2006; Liovic \& Lakehal 2007b). The advection scheme is based on second-order central differencing, and features flux-limiting to ensure monotonicity where necessary. Augmentation with VOF fluxes for computing flux densities is essential for consistency in the vicinity of the interface. Surface tension is modelled using the continuum surface force (CSF) model (Brackbill, Kothe \& Zemach 1992), with additional features such as height function-based curvatures for improved local surface tension estimates. These additional details for ameliorating curvature error were found vitally important for the modelling of SGS surface tension.

\subsection{Multi-physics treatment near deformable interfaces}

The multi-physics treatment near deformable interfaces to facilitate the implementation of (4.11), detailed by Liovic \& Lakehal (2007b), consists of two major steps: (i) generation of a distance function for the proximity to the interface from raw VOF colour function data and (ii) extraction of the friction velocity at the interface necessary to determine the non-dimensional interface turbulence length scale $y_{\text {Int }}^{+}$. Algorithms for generating an RDF are a means of extracting a distance function $\phi^{R D F}$ from VOF data. In the RDF algorithm used for the current work, the piecewise planar interface reconstructions of the centroid-vertex triangle-normal averaging (CVTNA) scheme for 3D VOF (Liovic et al. 2006) are used to generate interface markers by extracting the coordinates of all intercepts and centroids of interface planes within interface mesh cells. The reconstructed interfacial shear velocity (RISV) scheme (Liovic \& Lakehal 2007b) is then activated to locally determine the interface turbulence unit length scale $y_{I n t}^{+}$. Briefly summarizing, given a distance function distribution $\phi^{R D F}$, a mesh sweep confined to a one-cell interface support is performed, in which a new list of interface points is identified and the shear recorded. Cell-centred points $(P)$ are identified to the gas side of $\phi=0$ (or liquid side for liquidsided damping), and a normal estimate $\left(\hat{n}_{P}\right)$ is determined at that point. Tracing back along $\hat{n}_{P}$ generates an estimate of the extrapolated interface velocity and shear 
(a)

$\begin{array}{ll}\text { Bed angle } \beta & 1.64^{\circ} \\ \text { Quiescent bath depth } d_{0} & 0.4 \mathrm{~m} \\ \text { Deep-water wave amplitude } H_{0} & 0.12 \mathrm{~m} \\ \text { Wave period } T & 2.0 \mathrm{~s} \\ \text { Gravity } g & 9.81 \mathrm{~m} \mathrm{~s}^{-2}, \\ (b) & 30.0 \mathrm{~m} \times 2.0 \mathrm{~m} \\ \text { Domain size } & 2048 \times 130 \\ \text { Mesh size } & \delta x_{\text {normal }}=0.0146 \mathrm{~m} \\ \text { Mesh spacing } & \delta z_{\text {normal }}=0.0065 \mathrm{~m} \\ & \left(X_{o 2 L}, Z_{o 2 L}\right)=(10 \mathrm{~m}, 0 \mathrm{~m}) \\ \text { Offshore bed } & \left(X_{o 2 R}, Z_{o 2 R}\right)=(30 \mathrm{~m}, 0.5714 \mathrm{~m})\end{array}$

TABLE 1. (a) Input parameters of the cnoidal wave theory for the offshore wave generator (Goring 1979) and (b) mesh set-up and free-surface initialization, for the set-up of the TK96 wave-breaking simulation.

$\tau_{\text {Int }}$. The air-sided shear velocity $U_{\text {Int }}^{\tau}=\sqrt{\tau_{\text {Int }} / \rho_{G}}$ is computed to finally determine the interface turbulence length scale $y_{\text {Int }}^{+}$. The LEIS modelling strategy as described above has been implemented in the CMFD code TransAT of ASCOMP, and is being used for various practical interfacial flow problems.

\section{LES of short spilling water waves}

We first consider the breaking of shorter waves for which extensive experimental data are available. The collection of results from the experiments conducted by Ting \& Kirby (1996, hereafter TK96), specifically the phase-averaged data for spilling breakers, are well known and have been widely used elsewhere for validation of breaking wave simulations (e.g. Lin \& Liu 1998). We use the same data for validation purposes and for preliminary identification of differences between two-dimensional and fully three-dimensional simulation results.

\subsection{Two-dimensional simulations}

\subsubsection{Problem set-up}

Numerical investigation of the TK96 experiment is performed here initially in two dimensions. The set-up involves a vertically downward gravity contribution and a bed angle $\beta$ corresponding to the $1 / 35$ bed slope imposed in the experiments of TK 96 . The immersed-boundary internal obstacle treatment (Fadlun et al. 2000) is applied to mimic the upward-sloping seabed. Table $1(a)$ summarizes the details of the TK96 experiment, while table $1(b)$ specifies the geometry initialized in the simulation to match the experiment. The $30 \mathrm{~m}$ long computational domain is moderately truncated as compared with the experiment (which was based on a $40 \mathrm{~m}$ tank), but is long enough to prevent possible wave reflections that may affect ensemble-averaged results. For the purpose of a quantitative comparison with experiment, the employed grid resolution $(2048 \times 130)$ is sufficient in both spatial directions. The free surface is initialized as quiescent and waves are generated using the cnoidal wave generator introduced by Goring (1979). Care is taken in wave generation to verify that net liquid accumulations in the flow domain over time are zero, i.e. in a wave period, the sum of inflows equals the sum of outflows. 


\subsubsection{Phase-average profiles}

The extension of LEIS from $2 \mathrm{D}$ to $3 \mathrm{D}$ to capture interface-turbulence interactions associated with wave-breaking transients represents a significant upgrade in computational complexity. Furthermore, performing 3D LEIS for long enough to achieve ergodic turbulence statistics will remain a major task in the near future, and this is the case here, too. Prior to the TK96 experiments, Ting \& Kirby (1994) introduced a relative deviation statistics based on $N=102$ wave periods to analyse the effect on the averaged data with varying the number of periods; they found that the variations for only $N<10$ were the most significant. In their $2 \mathrm{D}$ simulation of a strong plunging breaker (based on the data of Ting \& Kirby 1995), Watanabe et al. (2005) performed phase-averaging over 30 periods, which allowed them to infer firstand second-order statistical moments. Alternatively, Lin \& Liu (1998) demonstrated reasonably good agreement in 2D simulation through considering only one wave period (instead of a multi-wave ensemble), using the standard $k-\epsilon$ model.

Within this preliminary validation phase, the effect of phase-averaging on the results has been investigated by incrementally increasing sample sizes. This is sought to understand the level of inference that can be undertaken with limited-size ensembles, in particular for 3D LEIS campaigns where only $O\left(10^{1}\right)$ wave period ensembles are currently feasible. Christensen (2006) have shown that seven periods were sufficient to reproduce turbulence statistics close to the experiment. The current work tests this assertion through the simulation of 25 wave periods, of which the first 10 accounting for flow development, wave propagation and breaking are are left offstatistics. Only the subsequent 15 wave periods are in effect used for ensemble phase-averaging. Figure 6.1.2 shows the phase-averaged interface elevation, $u$ - and $w$ - velocity components and TKE profiles. The plots result from averaging over an increasing number of wave periods. The comparison is made to corroborate with the $\left(x-x_{b}\right) / h_{b}=4.397$ streamwise locations in the TK96 experiment, using the $(z-\bar{z}) / h=-0.2623$ and $(z-\bar{z}) / h=-0.9080$ points along that crossflow location the extremes of vertical location in the offshore planes measured in the TK96 experiments.

The phase-averaged simulation results presented in figure 1 show very good comparison with the data. The free-surface elevation features a slight underestimation that reduces over time. This result is useful not only for showing convergence to the experimental result from the TK96 data set but also for showing that the wave profiles can be captured without attenuation. While the phase-averaged velocities are similarly shown to converge towards the data, TKE profiles exhibit deviations from experiment, partly because of the use of fewer periods for ensemble-averaging (15) as compared with the experiment (100). The other source of discrepancy is associated with use of 2D simulation which necessarily misses 3D turbulence effects. Nevertheless, the TKE plots show reasonable correspondence in magnitudes including the peak values. The plots also suggest that the TKE signal stabilizes in fully developed wave-breaking flow.

\subsubsection{A priori evaluation}

The analysis of unresolved interfacial scales compared with unresolved turbulence scales conducted hitherto (Labourasse et al. 2007; Liovic \& Lakehal 2007b) is also worth reconsidering in the present $2 \mathrm{D}$ simulations of the TK96 problem. A sample flow field after 11 wave periods is chosen and assumed to be a DNS flow field; a motivation for using a flow field from fully developed wave breaking is because it features both 2D representations of bubble entrainment and spray droplet formation, 

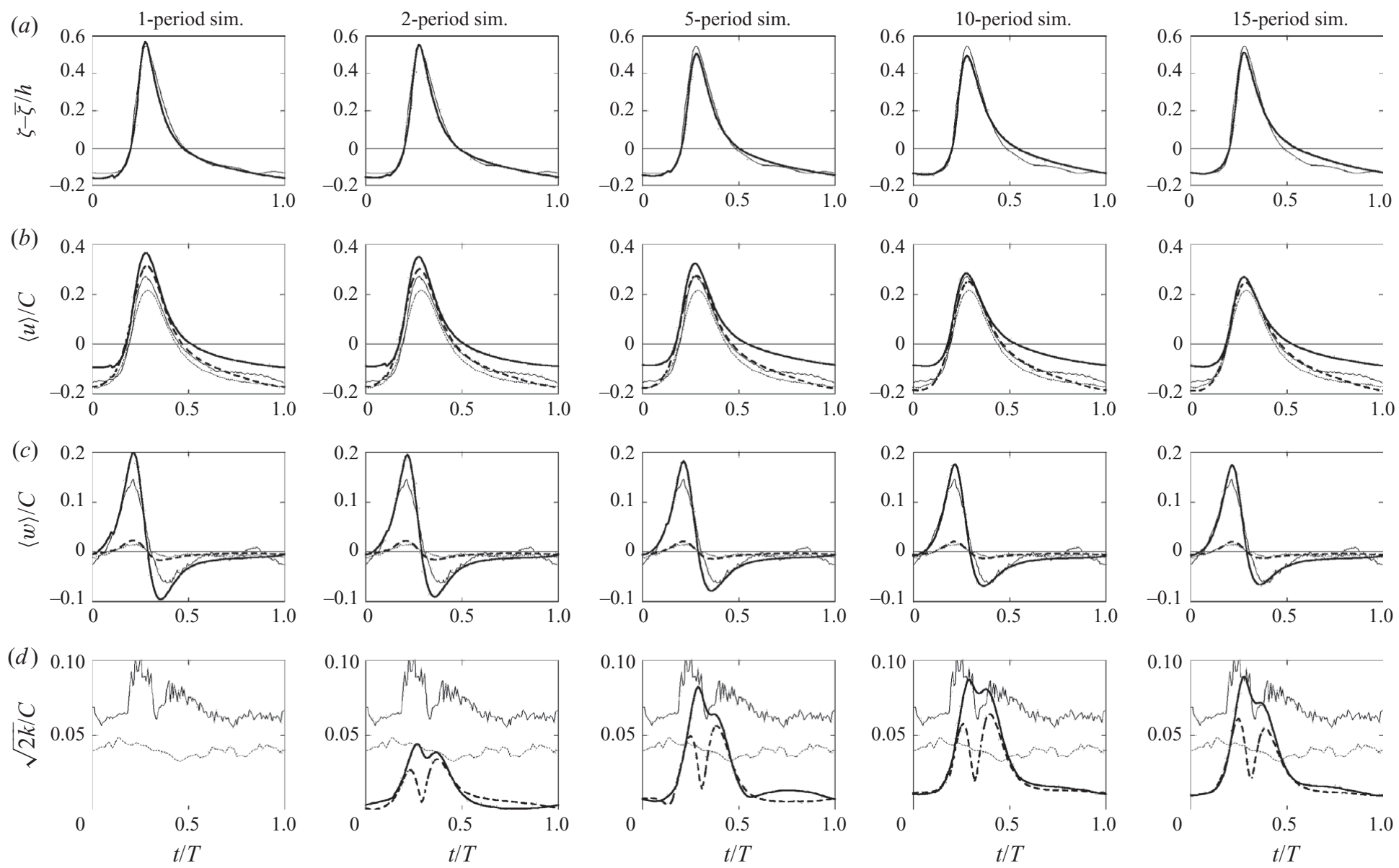

FIGURE 1. Change in free-surface elevation and velocity-based statistics in fully developed short-wave breaking as a function of the number of periods used for phase-averaging of the 2D simulation results of the Ting \& Kirby (1996) experiment on a $2048 \times 130$ mesh. Free-surface level at $\left(x-x_{b}\right) / h_{b}=4.397(a)$ : __ simulation; — experiment; $u$-velocity $(b), w$-velocity $(c) ; T K E(d),\left(x-x_{b}\right) / h_{b}=4.397$ and $(z-\bar{\zeta}) / h=-0.2623$ :

- - simulation; ------, experiment; $\left(x-x_{b}\right) / h_{b}=4.397$ and $(z-\bar{\zeta}) / h=-0.9080:--$, simulation; ------, experiment. 
hence features the small interfacial length scales that raise the relative importance of the non-SGS stress terms resulting from filtering, as discussed previously in presenting the model. Unfiltered flow variables $\left(u_{i}, \kappa, \hat{n}_{i}\right)$ and correlations $\left(u_{i} u_{j}, \kappa \hat{n}_{i}\right)$ are first estimated from this flow field, then tophat filtered estimates of $\widetilde{\nabla \cdot \tau_{i j}}$ and $\widetilde{\varepsilon_{\gamma}}$ from (4.7) and (4.6) are generated. Ratios of the form

$$
r_{i}^{S G S}=\left|\widetilde{\varepsilon_{\gamma_{i}}}\right| / \sum_{k}\left|\widetilde{\frac{\partial \tau_{i k}}{\partial x_{k}}}\right|, \quad i=1,2,
$$

explicitly show the importance of the SGS unresolved surface tension term relative to SGS turbulence stresses. Figure $2(a, b)$ compares the SGS turbulent stress and the unresolved surface tension in the $x$-momentum balance. The orders of magnitude difference is shown in figure 2(c), which reveals that the level of the SGS turbulent stress is generally one-to-two orders of magnitude larger than the unresolved surface tension. While another large part of the interfacial region exhibits SGS turbulent stresses being more than two orders of magnitude larger, only smaller localized regions feature levels of unresolved surface tension comparable with the SGS stresses. This evaluation justifies neglect of SGS modelling for unresolved surface tension in the current work.

\subsection{Three-dimensional simulation}

The results in figure 1 exhibit a good comparison with the experimental results of TK96, and validate the current numerical model for wave-breaking flows. Beyond the prevailing 2D wave motion, the effects of three-dimensionality in wave breaking are always observed in the interface kinematics of surf zones. Before proceeding to LEIS of steep breaking waves, the simulation of the TK96 spilling wave scenario has been extended to three dimensions. The same $2 \mathrm{D}$ set-up is used in the $(x, z)$ plane and a $y$-direction is added resulting in longshore extent $Y=0.6 \mathrm{~m}$. No-slip walls are subsequently added at $y=0$ and $y=0.6 \mathrm{~m}$ to mimic the experiment. The $3 \mathrm{D}$ computational domain is discretized by a $1024 \times 32 \times 128$ mesh - a 4.2 million cell mesh that is arguably quite large for long-time LEIS for rigorous ensemble averaging, but sufficient for single-period investigations of the breaking event. The same cnoidal wave generator is used in the $3 \mathrm{D}$ case.

Figure 3 shows a snapshot of the $C=0.5$ isosurface (indicating mean free-surface location) generated at instant $t=13 \mathrm{~s}$ (the seventh wave period). The waves feature dimpling in the longshore direction, and the free surface is still wrinkled due to the aftermath of the preceding breaking events even in the absence of waves. These mechanisms will be thoroughly discussed in the context of steeper breaking waves discussed next. The transition from $2 \mathrm{D}$ to $3 \mathrm{D}$ wrinkling in the free-surface approaching shore is an indicator of three-dimensionality. Analysis of the velocity field in the nearshore flow cross-section shows isolated counter-rotating vortices developing in the vicinity of the deformed interface.

\section{LEIS of steep breaking waves}

We now consider the interesting case of steeper breaking waves, featuring waves of higher amplitude. With higher waves, the width of the time-averaged interfacial zone $(0<\bar{C}<1)$ is made wider than for wave-breaking scenarios featuring lower-amplitude waves (TK96). 
(a)

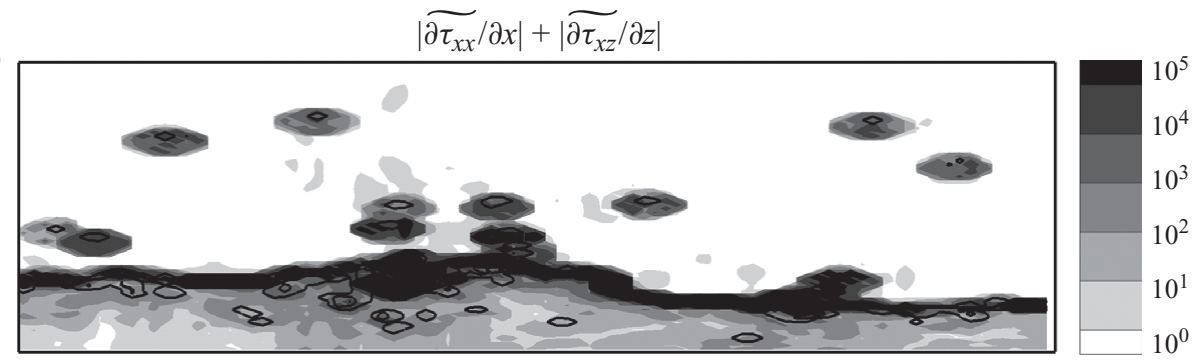

(b)

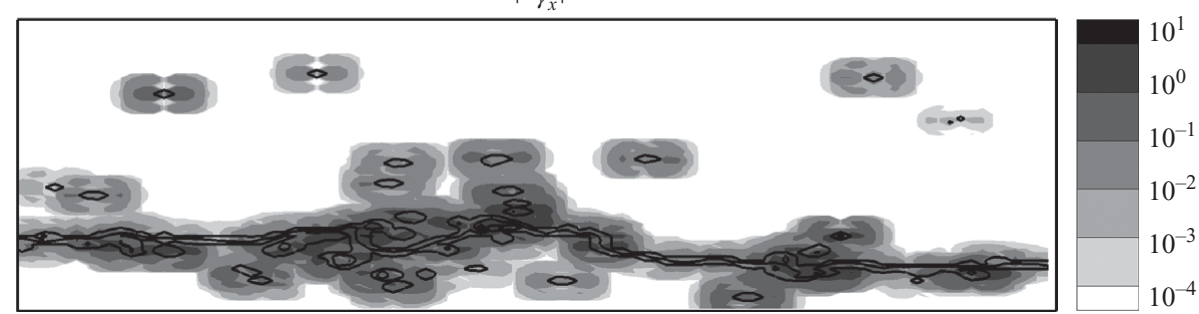

(c)

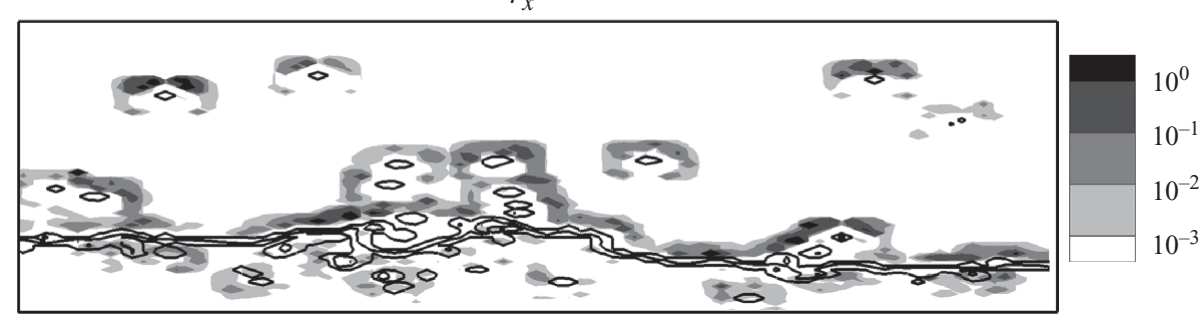

(d)

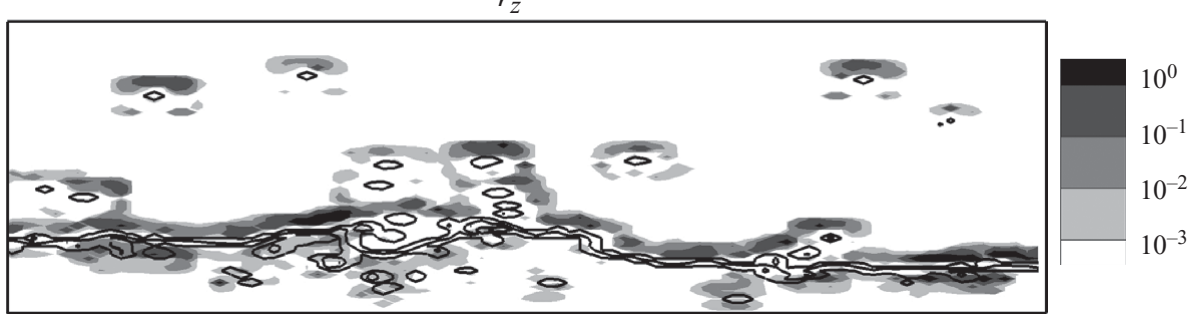

FIGURE 2. Results of a priori evaluation (Liovic \& Lakehal 2007b) applied to an instantaneous flow field from the 2D TK96 simulation: (a) maximum magnitude of the SGS stress for the update of $u ;(b)$ magnitude of unresolved surface tension for the update of $u ;(c)$ ratio of unresolved surface tension to SGS stress for the update of $u ;(d)$ ratio of unresolved surface tension to SGS stress for the update of $w$. The plots are rotated $\beta$ anticlockwise to reflect the slope of the seabed and are focused on the subdomain $16.0 \geqslant x \geqslant 18.5,0 \geqslant z \geqslant 0.7$.

\subsection{Problem set-up}

A constant bed slope $\beta$ and off-vertical gravity contributions are used in the problem set-up depicted in figure 4 . The bottom boundary of the computational domain coincides with the seabed, for increased accuracy (as compared to immersed boundary methods). The dimensions of the solution domain are chosen to achieve some 


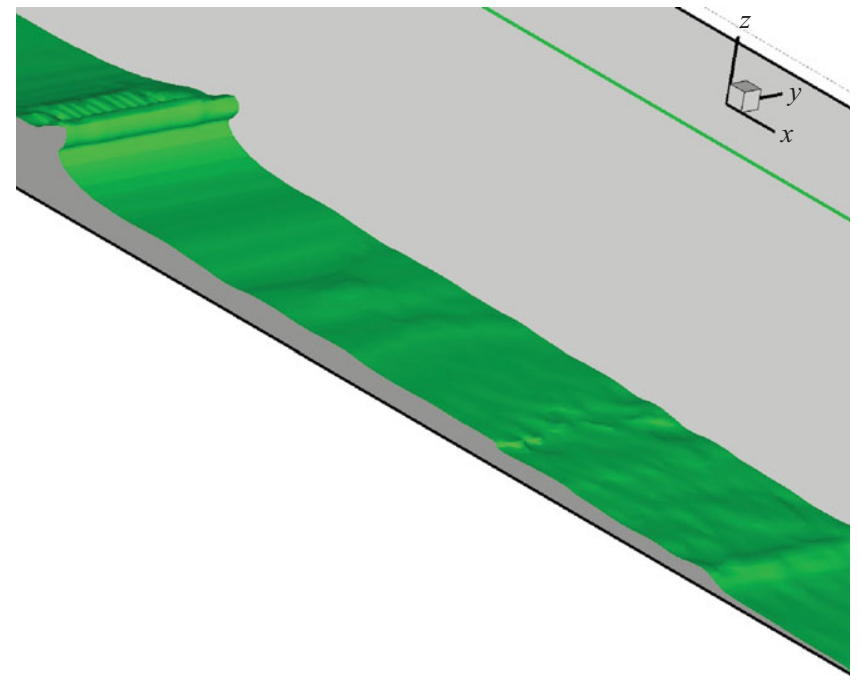

FiguRE 3. (Colour online) Snapshots of fully developed wave breaking from 3D simulation of the Ting \& Kirby (1996) experiment on a $1024 \times 32 \times 128$ mesh $(t=13 \mathrm{~s})$, showing 3D wrinkling with wrinkles being generated perpendicular to the shoreline.

similarity to the problem initialization used by Christensen \& Deigaard (2001), and are summarized in table 2 . A fine grid resolution in the direction perpendicular to the mean free surface is important in resolving viscous sublayers down to $y_{\text {wall }}^{+} \approx y_{\text {Int }}^{+} \approx 0.1$.

Unlike pressure-gradient-driven flows, the breaking wave flow is initially at rest, developing subsequently under gravity and the loading imposed by the wave generator. A rough estimate of the 'potential' flow Reynolds number based on the mean depth $d$ of the 'potential' liquid flow is $R e_{d}^{p o t} \approx 140$. Once the potential energy of the liquid is transformed into kinetic energy, the actual Reynolds number should, over time, adapt accordingly. The fluctuating turbulent field initially imposed was generated using a Gaussian distribution with zero mean and a standard deviation of $\sigma_{u}=\sqrt{g d \sin (\beta)}$. After solutions representative of the fully developed flow were obtained, the shear Reynolds number was found to converge in both phases towards $R e_{I n t}^{\tau}=U_{I n t}^{\tau} d / v \approx 400$, where $U_{I n t}^{\tau}$ is the friction velocity (equally applicable from both sides of the interface). The fifth-order Stokes theory solution of Fenton (1985) is the basis of the free-surface initialization and boundary conditions employed here. For the purpose, we choose parameter values based on the 'weak plunger' case study of $\mathrm{CD}$, though featuring significantly higher wave amplitude. These parameters also listed in table 2 - are used as inputs for the wave generation and initialization based on the theory of Fenton (1985). The table also includes the resulting values of commonly used wave-breaking characterization parameters.

The literature on wave breaking indicates that spilling breakers are associated with low bed-slopes and higher wave-heights, whereas plunging breakers are associated with higher bed-slopes and lower wave-heights. The wave set-up for the present study should result in spilling breakers, since $\varepsilon_{0}$ drops below the transition value of 0.5 . The experimental study of Lin \& Hwung (1992) has shown 'weak plunger' breaking behaviour even for $\varepsilon_{0}<0.5$, and reaffirms the fact that the criterion proposed by Galvin (1968) and Battjes (1975) (see (4.8)) is only an approximate guideline. With the weak plunger behaviour seen for the lower wave-height configuration, spilling 


$\begin{array}{ll}\text { Bed angle } \beta & 4.23^{\circ} \\ \text { Bath depth at boundary } d & 0.321 \mathrm{~m} \\ \text { Deep-water wave amplitude } H_{0} & 0.12 \mathrm{~m} \\ \text { Wave period } T & 1.4 \mathrm{~s} \\ \text { Stokes drift velocity } c_{S} & 0 \mathrm{~m} \mathrm{~s}^{-1} \\ \text { Gravity } g & 9.81 \mathrm{~m} \mathrm{~s}^{-2} \\ \text { Wavenumber } k & 2.85 \mathrm{~m}^{-1} \\ \text { Celerity } C & 1.57 \mathrm{~m} \mathrm{~s}^{-1} \\ \text { Wavelength } L_{0} & 2.21 \mathrm{~m} \\ \text { Deep water steepness } H_{0} / L_{0} & 0.0544 \\ \text { Surf-similarity parameter } \epsilon_{0} & 0.32 \\ \text { Domain size } & 8.0 \mathrm{~m} \times 0.6 \mathrm{~m} \\ \text { Mesh size 2D wave flow } & 200 \times 80 \\ \text { Mesh spacing 2D wave flow } & \delta x=0.04 \mathrm{~m} \\ & \delta z=0.0075 \mathrm{~m} \\ \text { Longshore mesh spacing 3D flow } & \delta y=\delta z \\ \text { Mesh size longshore direction 3D flow } & 40 \\ \text { Domain size longshore direction 3D flow } & 0.3 \mathrm{~m}\end{array}$

TABLE 2. Input parameters of the fifth-order Stokes theory for the offshore wave generator (Fenton 1985) and mesh set-up, for the set-up of the steep (increased wave height) wave-breaking simulation.

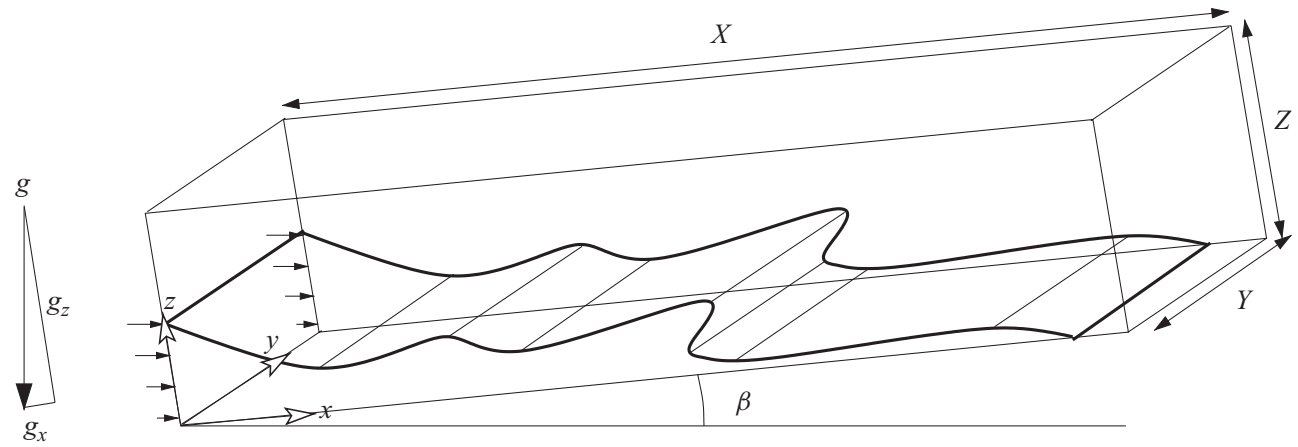

FIGURE 4. Problem set-up for the steep wave-breaking problem, showing the definition of key geometry and initialization parameters.

behaviour may also be expected here as waves are propagating shoreward and perceiving bottom earlier and further offshore.

\subsection{Boundary conditions}

The offshore boundary is prescribed as a fixed flow condition, with velocities determined as partial derivatives of the field potential based on the prescription of Fenton (1985) and the scaling procedure proposed by Christensen \& Deigaard (2001). The velocity distribution of air entering the solution domain at the offshore boundary is initialized to share the velocity of the water at the freesurface level, thus minimizing shear at the interface/offshore-boundary junction. This initialization presumes the water-sided wave generator to be responsible for near-surface currents on the air side of the free surface. Imposing wind to modify the wave-breaking phenomena is avoided. The onshore boundary is treated as an open boundary condition to ensure global mass conservation. The bed is represented using 
no-slip boundary conditions with the higher resolution resulting in low-Re treatment sufficient to avoid resorting to wall functions. Eddy viscosity is also computed near the seabed in the form of (4.10), but in combination with the original van Driest wall damping function. The upper boundary is treated as a free-slip boundary. The boundaries normal to the longshore direction are represented as periodic boundaries as done in the CD case, and unlike the no-slip walls at $y=0$ and $y=Y$ in the $3 \mathrm{D}$ TK96 simulation.

\subsection{Mesh size for LEIS of steep wave breaking}

As with any LES, sufficient mesh refinement is required to ensure that all supergridscale flow structures and interfacial details are captured, and achieving thorough mitigation of artificial diffusion. For the current steep wave-breaking scenario, a mesh dependence study is performed in order to establish minimal mesh size and computational resources required for 3D LEIS. For the purpose of assessing mesh dependence, a 2D study using the $x z$-plane set-up described in figure 4 and table 2 is conducted. Use of $2 \mathrm{D}$ instead of $3 \mathrm{D}$ is necessary for assessing mesh dependence a priori to a 3D study, and is indeed superior in that longshore fluctuations are eliminated. As such, mesh dependence can be established in one wave period, rather than using ensemble-averaged results dominated by sampling error in three dimensions. In the mesh-dependence study, successive refinement of mesh spacing from $(\delta x, \delta z)=(0.16,0.03)$ down to $(\delta x, \delta z)=(0.02,0.00375)$ is used to simulate two periods of $2 \mathrm{D}$ wave breaking. In the first instance, the mesh-dependence study consists of comparisons of time signals for free-surface height and velocity.

Figure 5 clearly demonstrates convergence of time signals with increased mesh refinement. Free-surface wave shapes and time signals feature greater variation in space and time for peaks as compared with troughs. Initial comparisons based on time signal tracking within the troughs show visual separation between the signal from the $(\delta x, \delta z)=(0.02,0.00375)$ simulation, and those for $(\delta x, \delta z)=(0.16,0.03)$ and $(\delta x, \delta z)=(0.08,0.015)$ - suggesting that these two coarser mesh resolutions are inadequate for LEIS. The next level of mesh refinement to $(\delta x, \delta z)=(0.04,0.0075)$ does not show any noteworthy differences in signal behaviour over larger stretches of signal recording. Peak capturing by the simulation on the second-finest mesh does result in non-negligible discrepancies. As an example, figure 6 shows differences in free-surface level just as wave turnover (from a propagating to a breaking wave) starts to occur. The clear attempted resolution of the peaks indicates that improving mesh resolution does not change the macroscale features of the flow, and is indicative of a spatial filter scale within the self-similar mesoscales (in terms of both turbulence and interfacial structures).

To determine whether the second-finest mesh resolution $(\delta x, \delta z)=(0.04,0.0075)$ is a potential cap on mesh resolution required to achieve LEIS of wave breaking, a comparison of extrema in peak and trough levels is also made. Table 3 shows minimum trough and maximum peak free-surface levels for given distances from the offshore wave generator. Multiple entries in table 3 show the convergence of the free-surface level already at the second-finest resolution, then the results from the finest-mesh resolution vary in another direction. This is a particularly strong signal that most of the flow solution is adequately resolved using the $(\delta x, \delta z)=(0.04,0.0075)$ mesh spacing, and that consideration of finer meshes is not necessary for meaningful physically realistic LEIS. 

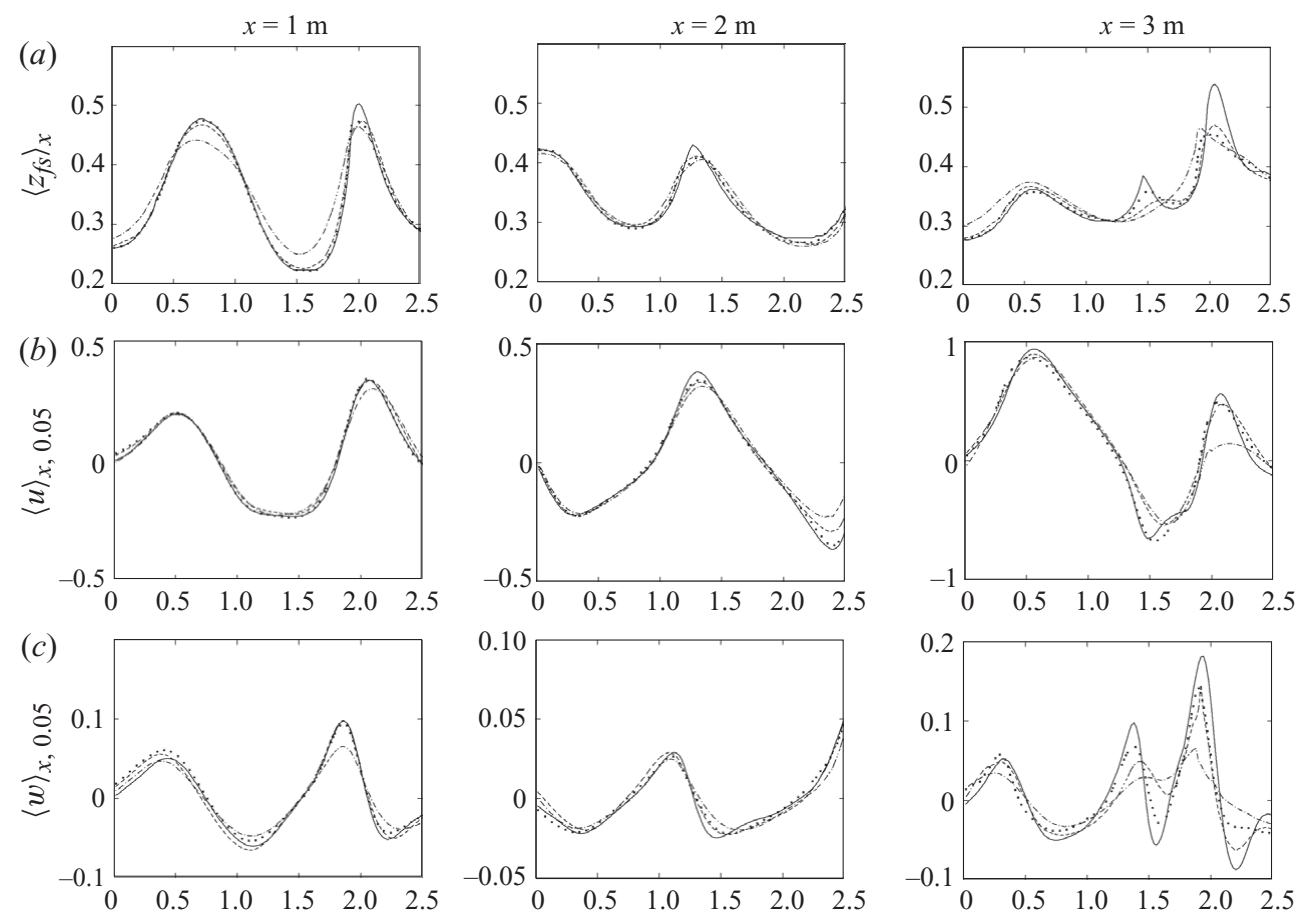

FIGURE 5. Time signals for 3D mesh-dependence study of steep wave breaking: $(a)$ free-surface level $z_{f s}$ at $x=1,2,3 \mathrm{~m} ;(b)\langle u\rangle$ at $(1,0.05),(2,0.05),(3,0.05) ;(c)\langle w\rangle$ at $(1,0.05),(2,0.05)$, $(3,0.05)$. Time signals are shown for simulations using mesh cell sizes: -.---, $\delta x=0.16 \mathrm{~m}$, $\delta z=0.03 \mathrm{~m} ;---, \delta z=0.015 \mathrm{~m} ; \cdots, \delta x=0.04 \mathrm{~m}, \delta z=0.0075 \mathrm{~m} ;-, \delta x=0.02 \mathrm{~m}, \delta z=0.00375 \mathrm{~m}$.

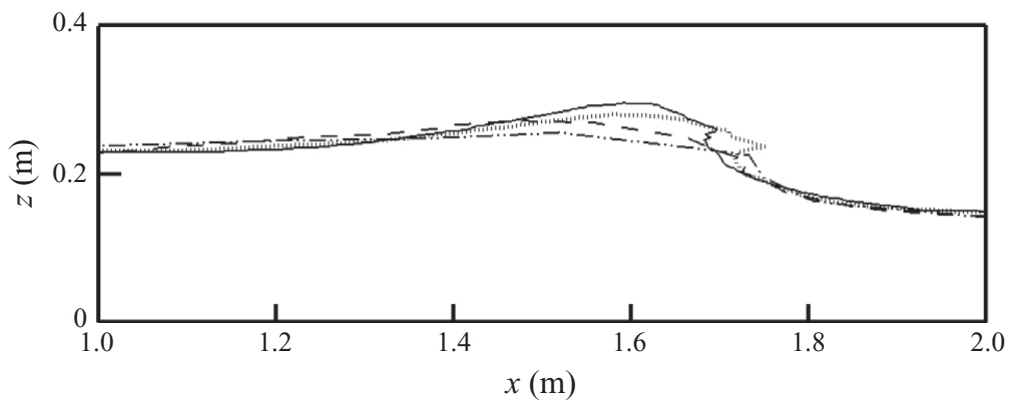

FiguRE 6. Changes in simulation-captured free-surface profile near maximum wave height prior to breaking at $t=1.2 \mathrm{~s}$ for varying degrees of mesh refinement. Free-surface profiles are shown for simulations using mesh cell sizes: -----, $\delta x=0.16 \mathrm{~m}, \delta z=0.03 \mathrm{~m} ;---, \delta x=0.08 \mathrm{~m}$, $\delta z=0.015 \mathrm{~m} ; \cdots, \delta x=0.04 \mathrm{~m}, \delta z=0.0075 \mathrm{~m} ;-, \delta x=0.02 \mathrm{~m}, \delta z=0.00375 \mathrm{~m}$.

\subsection{Validation results}

\subsubsection{Grid sensitivity}

Using $(\delta x, \delta z)$ from the second-finest mesh spacing considered, the 2D plane spanning the dominant $2 \mathrm{D}$ wave-breaking flow is resolved using $n x=200$ and $n z=80$. The third longshore direction is subsequently set to be $n y=40$. Figure 7 shows the time history of the free-surface elevation extracted at two locations along the mean 


\begin{tabular}{|c|c|c|c|c|c|c|}
\hline \multirow[b]{2}{*}{$(\delta x, \delta z)$} & \multicolumn{2}{|c|}{$x=1$} & \multicolumn{2}{|c|}{$x=2$} & \multicolumn{2}{|c|}{$x=3$} \\
\hline & $z_{\text {trough }_{1}}$ & $z_{\text {peak }}$ & $z_{\text {trough }_{1}}$ & $z_{\text {peak }_{1}}$ & $z_{\text {trough }}$ $_{1}$ & $z_{p e a}$ \\
\hline$(0.16$, & 0.2 & 0.371 & 7 & 0.353 & 4 & \\
\hline 10 & 0.263 & 0.383 & 0.298 & 0.355 & 0.305 & \\
\hline 075$)$ & 0.261 & 0.387 & 0.295 & 0.356 & 0.305 & 0.3 \\
\hline$(0.02,0.00375)$ & 0.262 & 0.388 & 0.296 & 0.365 & 0.305 & 0.3 \\
\hline
\end{tabular}

TABLE 3. Trough and peak levels above zero reference height (at depth $d=0.321 \mathrm{~m}$ ) for 2D steep wave-breaking simulation using different mesh resolutions.
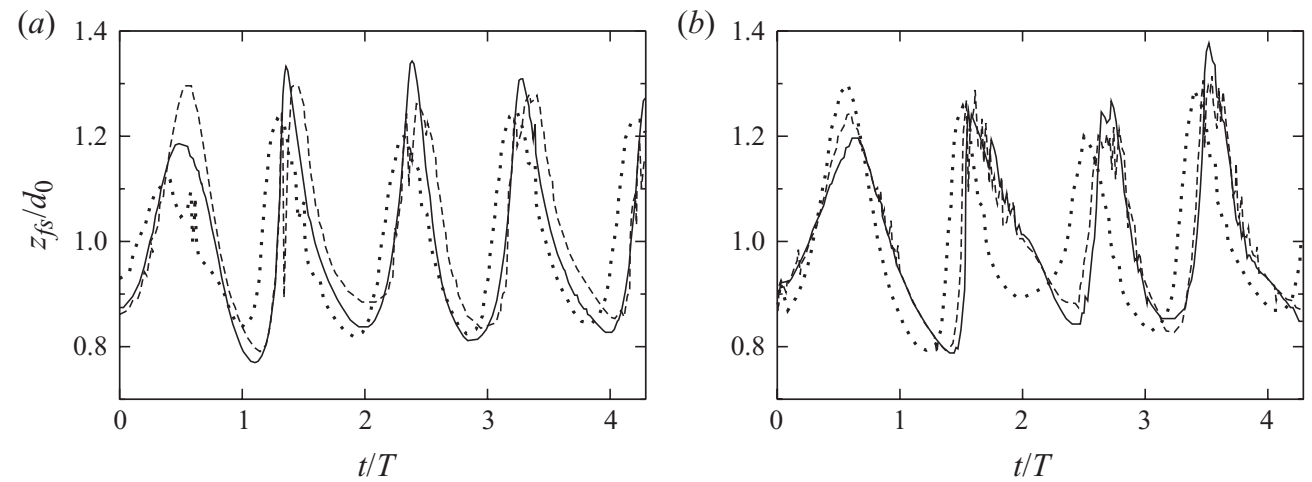

FIGURE 7. Time history and mesh dependence of the surface elevation for 3D steep wave-breaking flow at difference horizontal locations in the flow domain. The streamwise locations and seabed levels at those locations are (a) $\eta=10.4, z_{b} / d_{0}=0.23 ;(b) \eta=8.8$, $z_{b} / d_{0}=0.35$. The meshes corresponding to each signal: $\cdots, 50 \times 10 \times 20,---, 100 \times 20 \times 40$; ,$- 200 \times 40 \times 80$.

free-surface profile. The profiles show the free-surface evolution to feature variable degrees of nonlinearity. At more offshore locations, the signals feature the sustained periodicity characteristics of the flux boundary condition prescribed at the offshore boundary by the fifth-order Stokes theory. This behaviour is not sustained closer to shore, with less pronounced periodicity given surf-zone interactions between backwash and plungers. The steeper bed angle in this case results in the flow developing rather quickly after the first wave period, characterized by higher-amplitude crests and shallower troughs emanating from the offshore boundary. The noise occasionally affecting the profiles corresponds to the free surface ahead of the propagating steep wave crest, tilting beyond vertical and turning over during individual breaking events.

Taking the high-resolution simulation to be grid independent, the time signals at the more offshore locations in figure 7 show the extrema in the low-resolution simulations to be out of phase by $0.2 T-0.3 T$ - most of it introduced during start-up. The propagation of subsequent waves is much more accurate, with errors typically only about $0.05 T$ during a period. Crest heights and trough depths from successively refined meshes are not systematically under- or over-estimated, and the signals of the two higher-resolution simulations differ far less than those of the coarser simulations. These results indicate that the macroscopic features of the flow are adequately resolved, even if the LEIS does not resolve all of the interfacial length scales created by wave breaking. 


\subsubsection{Two-point correlations}

A normalized streamwise distance is introduced to facilitate interpretation of the results, namely $\eta=\left(X_{t r}-x\right) / d_{0}$, where $X_{t r}=4.35 \mathrm{~m}$ is the shore location of the sea under quiescent conditions relative to the origin (offshore boundary) of the computational domain, and $x$ is the streamwise coordinate relative to the origin ( $d_{0}$ in table 1). Two-point correlation analysis is often employed in LES and DNS studies of turbulent flows as a fundamental test of adequacy of the spanwise extent of the domain with regard to turbulence structures. The size of the structures is roughly twice the distance between the origin and the point at which the correlation levels off to zero. The domain is considered to be large enough in the periodic direction if all two-point correlations vanish at half the domain width. Only the $v$-velocity component is used for two-point correlation here; the $u$ - and $w$-velocity components are inappropriate for use because the dominance of the underlying $2 \mathrm{D}$ wave motion cannot be adequately decoupled from the turbulent 3D fluctuating field. The twopoint correlations have been determined between two points featuring the same $(x, z)$ coordinates:

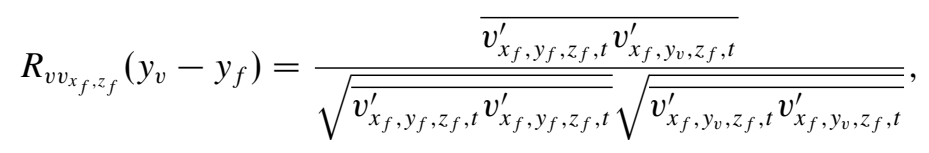

where $v_{x, y, z, t}^{\prime}=v_{x, y, z, t}-\bar{v}_{x, z}, v_{x, y, z, t}$ is the instantaneous velocity in the spanwise direction, and $\bar{v}_{x, z}$ is the time average of the spanwise-averaged quantity. In this representation, $\left(x_{f}, y_{f}, z_{f}, t\right)$ stands for the 'fixed' point, with $y_{f}=Y / 2$. The 'variable' point $\left(x_{f}, y_{v}, z_{f}, t\right)$ changes within the interval $0<y_{v}<Y$, where $Y$ is the total width of the domain.

The two-point correlation profiles $R_{v v}$ plotted in figure 8 show that the spanwise extent of the periodic computational domain is adequate for resolving the liquiddriven wave propagation and turbulence in the vicinity of the free surface. The correlation is shown not to vanish only for the case of $\eta=4.5$ and $z / d_{0}=0.93$. Energy spectra are steep at this air-side point, which (explained later on) indicates that the $2 \mathrm{D}$ motion associated with wave propagation is dominant and does not suggest that an important large scale of motion in the spanwise direction is being 'squeezed out'.

\subsubsection{Breaking index and characterization}

An important characteristic measure in wave breaking is the ratio of wave height to bath depth, $\gamma_{b}=H_{b} / d_{b}$, generally referred to as the breaker index. Wave height $H_{b}$ is measured as the vertical distance between the crest and the adjacent trough at breaking, while bath depth $d_{b}$ is based on the quiescent bath level. For the setup parameters in table 2, breaker index estimates quoted in the literature differ somewhat. For the CD weak plunger case, Christensen \& Deigaard (2001) cited the experimental values from Lin \& Hwung (1992) of $\gamma_{b}=0.99$, while their own simulations yielded $0.95-1.0$. It is worth noting, however, that the wave height in our case is significantly higher than the experiment of Lin \& Hwung (1992) and the simulation of Christensen \& Deigaard (2001). As such, alternative data sets and correlations need to be considered. On the basis of the problem set-up described in table 2, the selected data are listed below.

(i) Svendsen (1987). Here

$$
\gamma_{b}=1.05 S^{0.2}, \quad S=\frac{\tan (\beta)}{d / L_{0}} .
$$



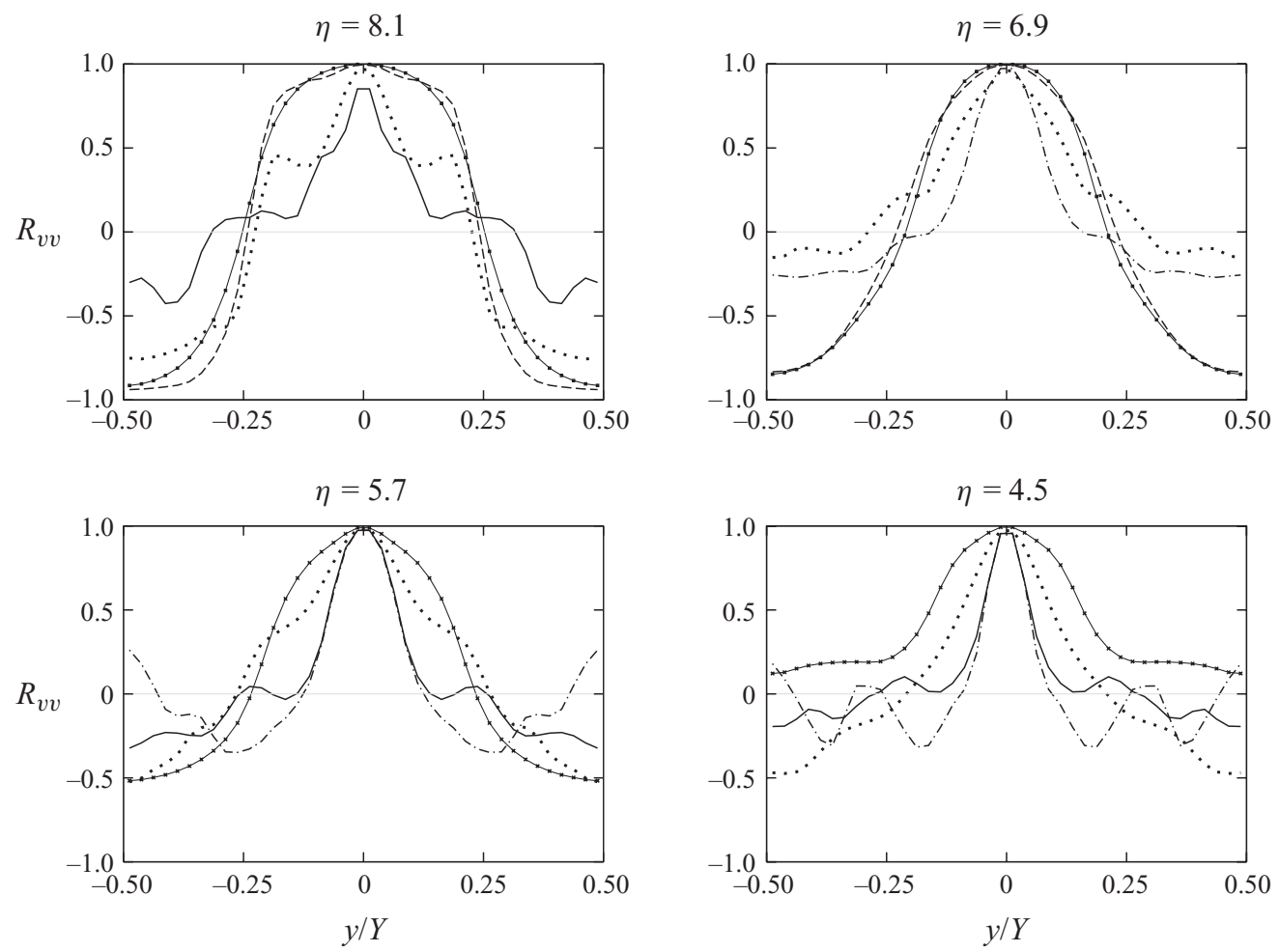

Figure 8. Two-point correlations at various $v$ locations in the flow:,$- z / d_{0}=0.15 ; \cdot-\cdot-$, $z / d_{0}=0.32 ; \cdots, z / d_{0}=0.48 ;--, z / d_{0}=0.62 ; \varkappa, z / d_{0}=0.78 ; ., z / d_{0}=1.09$.

This relation, where $S$ stands for the relative bottom slope, is commonly cited in the literature as a model for estimating breaker index. From the entries in table 2, $S=0.51$, such that the breaker index estimate is $\gamma_{b}=0.92$.

(ii) Smith \& Kraus (1991). The best-fit linear relation for the breaker index on plane slope beds was found to be

$$
\gamma_{b}=\frac{H_{b}}{d_{b}}=b(m)-a(m)\left(\frac{H_{0}}{L_{0}}\right),
$$

where $m$ is the bed slope and $H_{0} / L_{0}$ describes the wave steepness. In the formula, $a(m)=5.0\left(1-\mathrm{e}^{-43 m}\right), b(m)=1.12 /\left(1+\mathrm{e}^{-60 m}\right)$, and (7.3) was derived from multiple data sets for ranges of $0.0007 \geqslant H_{0} / L_{0} \geqslant 0.0921$ and $1 / 80 \geqslant m \geqslant 1 / 10$. For the set-up parameters in table 2, (7.3) yields $\gamma_{b}=0.85$.

(iii) Svendsen (2006). The formula

$$
\gamma_{b}=S^{0.25}
$$

is presented by Svendsen (2006) as being valid for $0.25<S<1$. Based on this formula, the breaker index estimate is $\gamma_{b}=0.84$.

(iv) Tsai et al. (2005). Experiments for a similar $1 / 10$ bed slope yield $\gamma_{b}=0.84$ for $S$ as high as 0.8. Extrapolation of their data set for low $S$ makes $\gamma_{b}<0.84$ for $S=0.51$.

In the present simulation, the breaking point occurs in the vicinity of $x=1.95 \mathrm{~m} \pm$ $0.10 \mathrm{~m}$, or $\eta \approx 7.4$, a location at which the resulting ratio $H_{b} / d_{b}$ is equal to 0.82 . The estimated breaker index obtained from simulation is within the error associated with the scatter of the experimental data sets and models listed above. For the current 
case, it is worth noting that the $50 \%$ higher wave height results in the propagating waves perceiving bottom further offshore in deeper water, thus resulting in values of $\gamma_{b}$ lower than those in Lin \& Hwung (1992) and Christensen \& Deigaard (2001). The result from the current simulation reproduces the experimentally observed breaker index well, and further validates the $3 \mathrm{D}$ model for steep wave breaking.

\subsection{Wave-breaking events}

The evolution and breakup of the waves over the course of the simulation is presented in figure 9 in the form of time frames of the $C=0.5$ isocontours marking the interface location. Snapshots during breaking show characteristics of both spilling and plunging breakers, and is still best described as 'weak plunging'. During start-up, the front face of the wave becomes vertical and the top of the wave turns over, indicative of plunging events. However, the jet thrown forward from the top of the front face does not feature significant momentum; the jet collapses to resemble spilling of a crest down the front of a wave, and no significant pipeline is formed. Later on in the developed flow (as shown in the sequence of snapshots in figure 9), there is a clear large-scale turning over of the wave, a more noticeable pipeline followed by air entrainment below the water surface - all features of plunging rather than spilling. In summary, the calculations show that the plunging event is weak because (i) the jet is not thrown forward far enough to form a pipeline of substantial radius and (ii) the splash-up forward of the impact point does not rise to a substantial fraction of the wave height.

\subsection{Form drag evolution}

Form drag in this case is expected to significantly change with the wave deformations at the head and tail of each breaker. The relationship between form drag and wavebreaking events is not well understood, even though these issues have been thoroughly tackled in other similar contexts (Calhoun \& Street 2001; Sullivan \& McWilliams 2002). For turbulent flow over a wavy surface studied by Calhoun \& Street (2001), pressure or form drag clearly dominates viscous drag contributions, at least for wave slopes of the order of $2 a / \lambda=0.1$. During wave breaking, the contributions of viscous drag on the surface are expected to be even less important compared with pressure drag, and consequently will not be discussed here. As noted by Calhoun \& Street (2001), the form drag can be presented in a number of different ways, depending whether the waves are static (as in flow over hill) or in motion; e.g. experiencing linear to exponential growth as in Lin et al. (2008). In this section, we investigate the variations in form or pressure drag during wave growth and plunging processes. Since the present flow is nominally driven by gravity and wave-generator forcing, airflow turbulence - via wind shear - is expected to be involved in wave birth only at the initial stage. During the later stage of the wave build-up and plunging, the processes should rather involve wind-induced pressure and stress fluctuations. This should be similar to that reported by Lin et al. (2008) in their DNS of wave formation, during exponential growth. The behaviour of pressure and shear stress fluctuations in the air is most probably wave dominated at the later stage. Pressure fluctuations at the surface in the present case are unlikely to play a direct role in wave birth or plunging, but are actually a result of the breaking process itself.

Local (not over an entire wave as in Sullivan \& McWilliams 2002) form drag distribution is determined here using

$$
D_{p}=p \hat{\boldsymbol{n}} A \cdot \boldsymbol{u}_{x}^{i n t} /\left|\boldsymbol{u}_{i n t}\right|,
$$



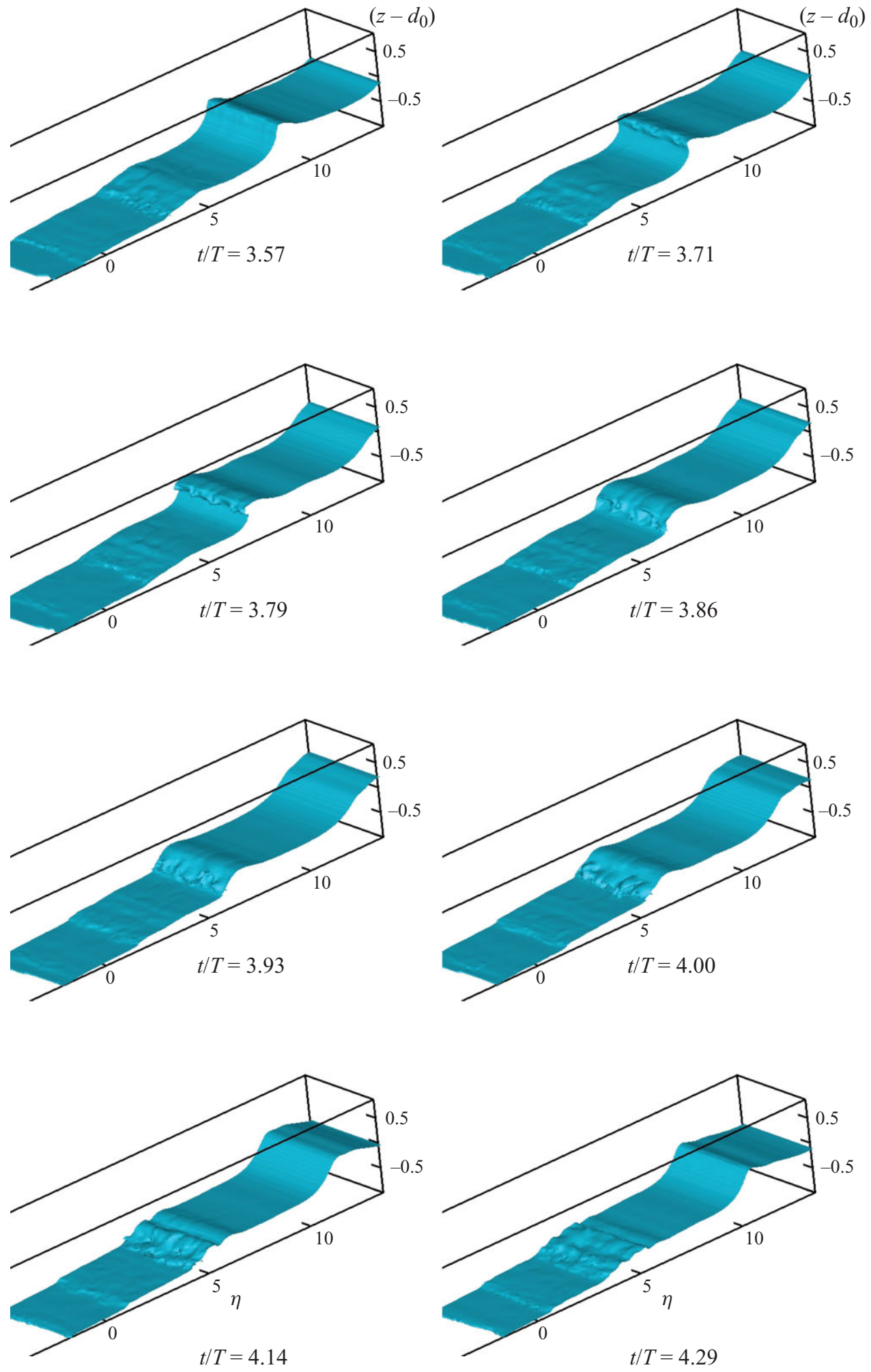

FIGURE 9. (Colour online) Free-surface profiles of wave breaking for the weak plunger scenario. The streamwise extent $0 \geqslant x \geqslant 4.5$ is shown, while the full extents are shown in the $y$ - and $z$-directions. 

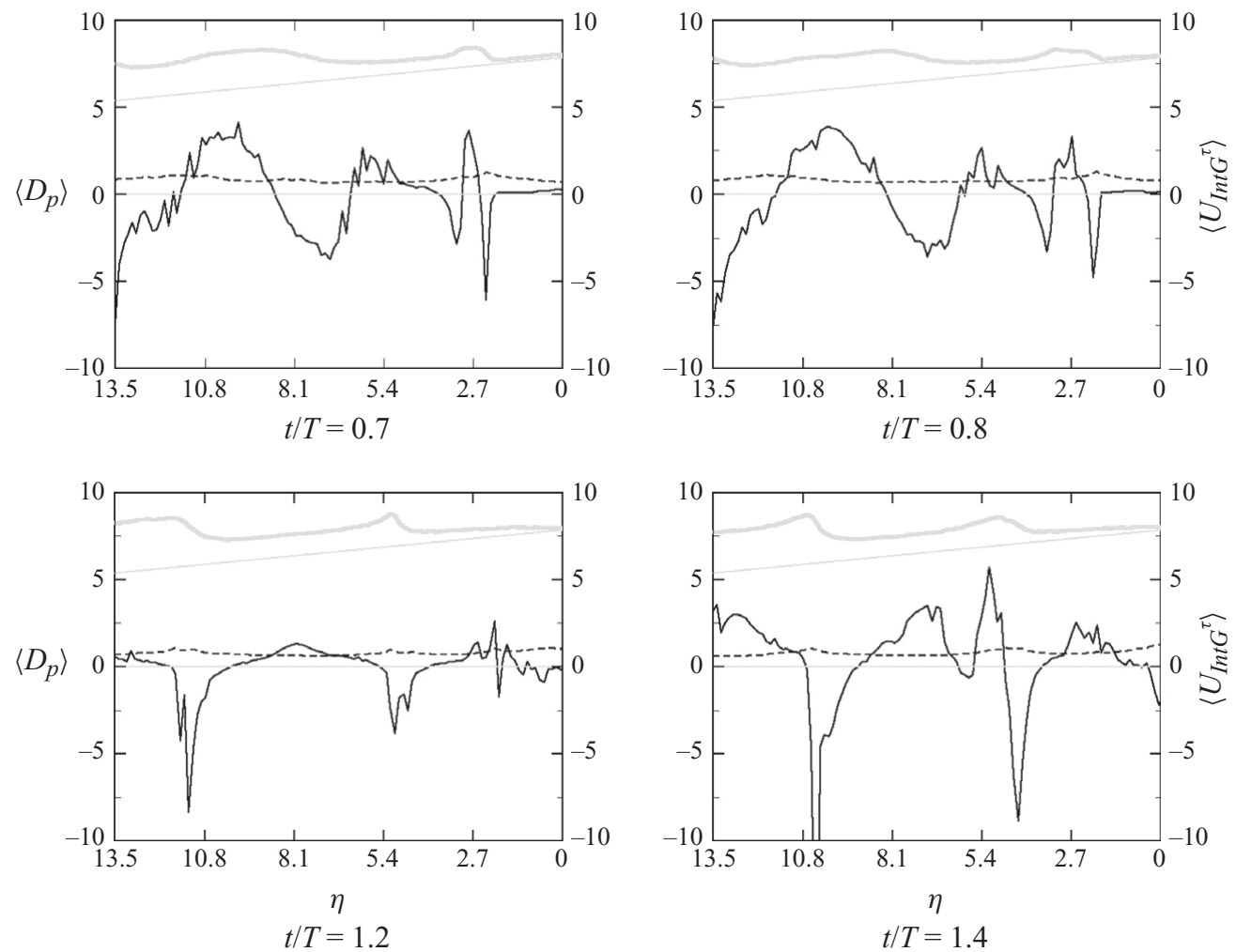

FIGURE 10. Spanwise-averaged form drag (-) and interfacial shear velocity (---) distributions along the wave propagation direction. The normalized streamwise coordinate $\eta=\left(X_{t r}-x\right) / d_{0}$ is introduced on the horizontal axes. The (longshore-averaged) free-surface shapes with elevations matching the streamwise coordinates are shown in grey, for matching of form drag with free-surface shape.

where $p$ is the surface pressure, $\hat{\boldsymbol{n}}$ is the unit normal to the interface, and $A$ is the local surface area. Here, we define the dimensional form stress per unit area as a projection of surface pressure onto the interface; we also recognize that other definitions are also possible. The unit vector $\boldsymbol{u}_{x}^{\text {int }} /|\boldsymbol{u}|$, where $\boldsymbol{u}_{x}^{\text {int }}$ denotes the horizontal component of the interface speed vector, is introduced as a measure of the underlying surface slope. The pressure acts not on surface segment $\mathrm{d} A$, but only on the part of $\mathrm{d} A$ that is perpendicular to the interface speed vector $\boldsymbol{u}^{i n t}$. Furthermore, $D_{p}$ is normalized by interfacial shear, justified by the fact that $U_{\tau}$ remains relatively constant, as shown on the right axis of figure 10. The first finding of this exercise is that stress fluctuations at the interface are rather passive in gravity and external forcing breaking processes. Because wave surface area depends on its wavelength and amplitude, these two quantities are introduced beside $U_{\tau}$ to normalize $D_{p}$. A low-order estimate of the surface area (per unit of spanwise length) is the hypotenuse. As such, the normalizing factor for $D_{p}$,

$$
f_{d}=\left(H_{0}^{2}+L_{0}^{2}\right) \rho_{G} \bar{U}_{I n t G}^{\tau}{ }^{2},
$$

takes the value of $f_{d}=2.07 \times 10^{-3}$, for $R e_{\text {Int }}^{\tau}=400$ (see table 2) and $\bar{U}_{\text {Int }}^{\tau}{ }^{2}=$ $0.019 \mathrm{~m} \mathrm{~s}^{-1}$. The normalized spanwise-averaged form drag and interfacial shear 
velocity distributions, defined respectively by

$$
\left\langle D_{p}\right\rangle=\frac{1}{z f_{d}} \int_{-a}^{a} D_{p} \mathrm{~d} z \quad \text { and } \quad\left\langle U_{\text {IntG }}^{\tau}\right\rangle=\frac{1}{{\overline{U_{I n t G}^{\tau}}}^{2} z} \int_{-a}^{a} U_{\text {Int }}^{\tau} \mathrm{d} z
$$

are plotted in figure 10 along the wave propagation direction. The scale for $\left\langle D_{p}\right\rangle$ is shown on the left $y$-coordinate axis, while that for $\left\langle U_{\text {IntG }}^{\tau}\right\rangle$ is shown on the right axis. The thick grey line shows the wave shape over the $x$-extent of the plots.

In the early stage of wave development, prior to breaking, there is a persistent trend for maximal negative form drag occurring ahead of wave crests, while positive form drag tends to be found along the section of low-curvature free surface between wave crests. This is equivalent to wave birth and growth being essentially linked with pressure drag over the surface. The profiles are almost in phase, featuring various modes with different amplitudes. When the pressure drag drops at peaks in the free-surface profiles, viscous shear becomes large. In the troughs, the pressure drag as expected tends towards zero, and the frictional shear drops to its minimum. Near-zero or slightly negative form drag corresponds to flow regions where the wave travels fast while maximum levels correspond to nearly standing-wave scenarios. These findings are in agreement with those reported by Calhoun \& Street (2001) in particular.

\section{Mean flow results}

We proceed in this section by extracting information about the transport in the mean flow, including the exchange of energy between the waves and the mean and vice versa. Intuitively speaking, wave steepening and breaking is a result of energy transfer from the mean flow to the unstable wave modes. Smallscale turbulence is understandably less important at this stage. Wave start-up is reasonably presumed to prevail till $t \approx 1.4 T$. Only beyond this point can the results actually be representative of a fully developed wave propagation and breaking flow.

\subsection{Averaged contour distributions}

We proceed by decomposing the flow into mean flow and unstable wave modes, as has been practiced for temporally or spatially evolving mixing layers (Narayanan \& Lakehal 2006). Data generated after this threshold $(\widetilde{\varphi})$ are first averaged spatially in the spanwise lateral direction (denoted by the symbol $\langle\varphi\rangle$ ), then over time to yield $\overline{\langle\varphi\rangle}\rangle$. Note that for simplicity we have omitted the tilde symbol. The difference between the spanwise average and the time average of the spanwise-averaged velocity $u_{i}^{\prime \prime} \equiv \overline{\left\langle u_{i}\right\rangle}-\left\langle u_{i}\right\rangle$ is designed to estimate the wave velocity, containing both the unstable modal velocity and small-scale fluctuations superposed on it, i.e. $u_{i}^{\prime \prime}=u_{i}^{m}+$ $u^{\prime}$. It should be stressed that in order to distinguish between the modal-induced and turbulence-induced energies, use should be made of ensemble averaging over various realizations: this is possible in experimental studies (e.g. Ting \& Kirby 1996), but rather difficult for the time being with expensive simulations of the LEIS sort.

Figure 11 shows averaged velocities and volume fraction, while figure 12 shows root-mean-square (r.m.s.) distributions. Time-averages here were generated over four wave periods: this is probably shorter than desirable for the purpose of generating ergodic flow statistics. The time-averages are not intended for the purpose of detailed 
(a)

(b)
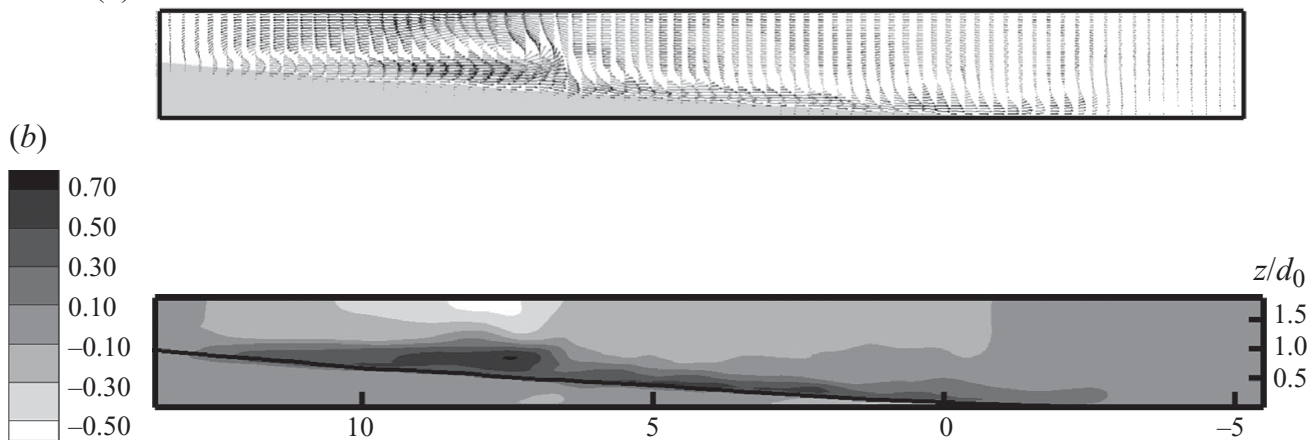

(c)

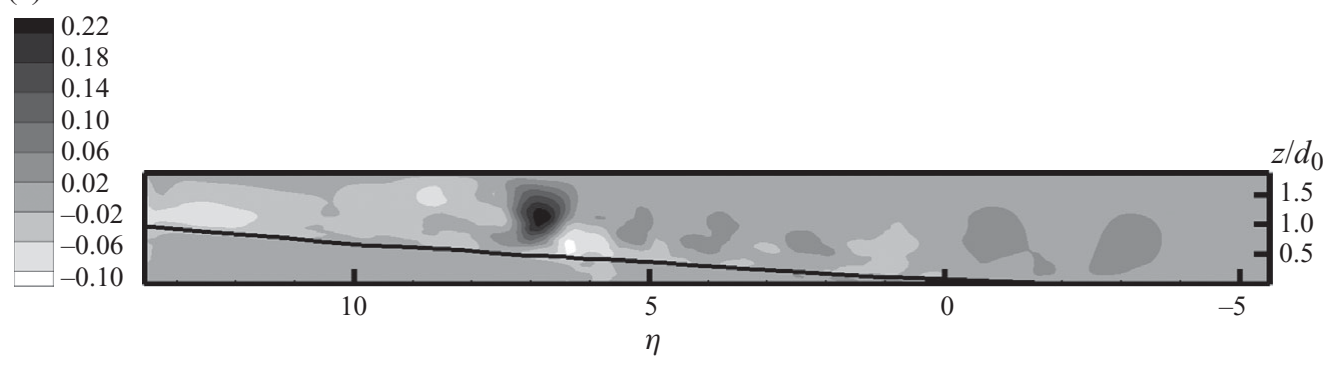

FIGURE 11. $(a-c)$ Longshore-averaged distributions $\langle c\rangle,\langle u\rangle$ and $\langle w\rangle$, respectively, based on time-averaging after $t=1.4 T$. The full extent in the (near-vertical) $z$-direction is shown, while the streamwise extent is $0 \geqslant x \geqslant 6.0 \mathrm{~m}$.

comparison with a specific set of wave-breaking data. Instead, the results presented in figures 11 and 12 help derive trends by reference to documented data. The velocity components in these figures are relative to the rotated reference frame (in which gravity is oriented away from vertical). As expected, the mean liquid flow corresponds to a quiescent surface solution. The averaged airflow solution above the free surface highlights however a strong shoreward current of air, and a similarly strong seaward counter-current shear layer above the shoreward zone. This indicates that surface topology reaches steady state faster than the airflow; the liquid mean flow also shows ergodicity after the simulated four waves. The result also shows how important it is to account for air entrainment by the waves in situations of high wave amplitude. The outcome of this statistical analysis of the flow suggests that care must be taken in interpreting the averaged profiles, valorizing rather the interpretation of the results in the context of time during which the waves grow, steepen and break. This is indeed done in $\S 9$.

A closer look at the reverse flow on top of the sheared surface suggests that in the breaking zone in particular, the velocity field has the typical shape of a jet flow associated with airflow entrainment. This observation is corroborated by the contours of the averaged streamwise velocities. The averaged crossflow velocities are one order of magnitude weaker than in the streamwise direction. In this breaking zone, the flow field is clearly dominated by streamwise stresses, the intensity of which seems to depend on interface proximity. The intensity of vertical and spanwise stresses marks the location of the core jet region. In the shoaling and breaking zones, the water-sided fluctuations in the spanwise velocity component $\sqrt{\overline{v^{\prime \prime} v^{\prime \prime}}}$ are smaller further 
(a)
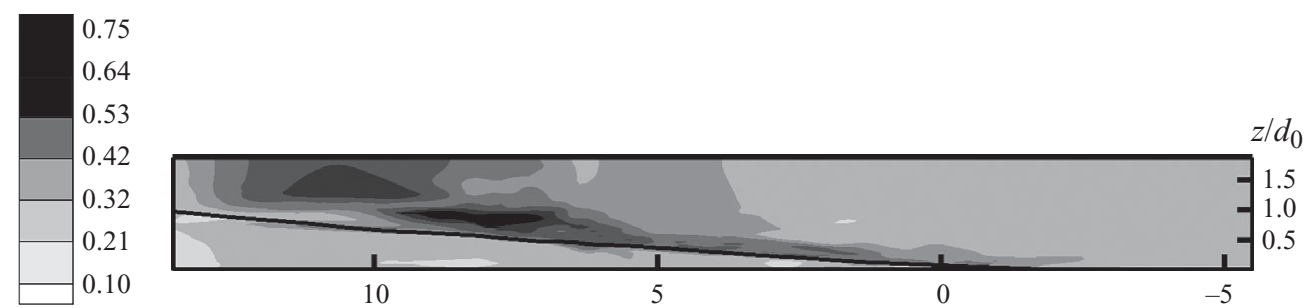

(b)
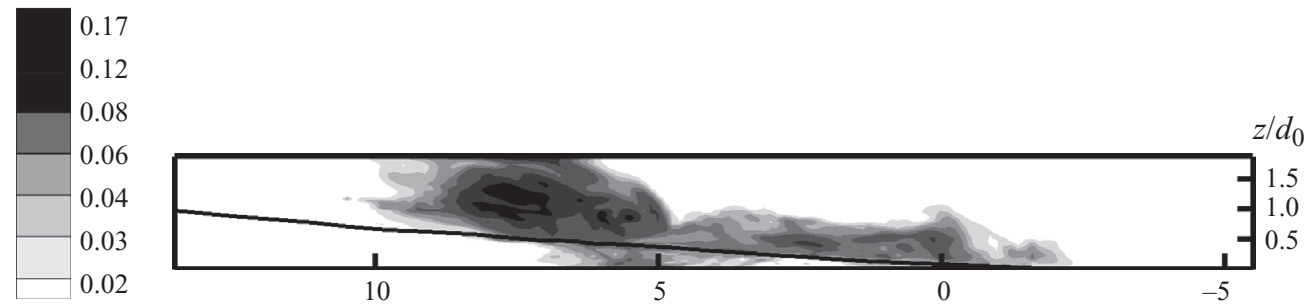

(c)
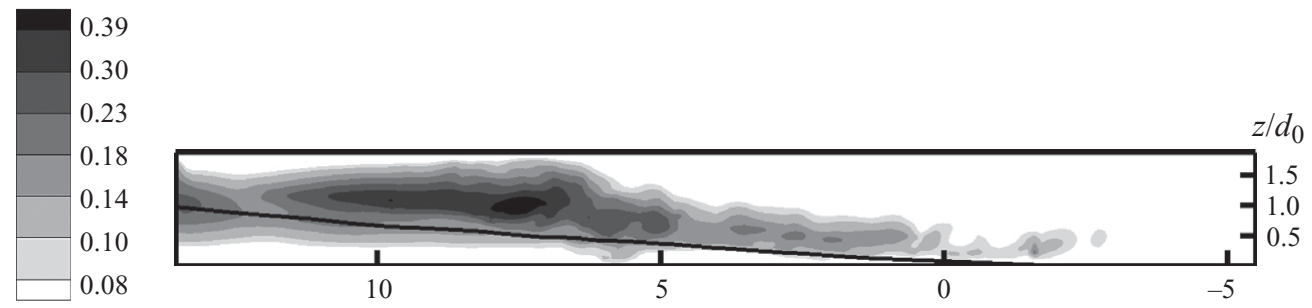

$\eta$

Figure 12. $(a-c)$ Longshore distributions of r.m.s. quantities $\left\langle\sqrt{u^{\prime \prime 2}}\right\rangle,\left\langle\sqrt{v^{\prime \prime 2}}\right\rangle$ and $\left\langle\sqrt{w^{\prime \prime 2}}\right\rangle$, based on time-averaging after $t=1.4 T$. The full extent in the (near-vertical) $z$-direction is shown, while the streamwise extent is $0 \geqslant x \geqslant 6.0 \mathrm{~m}$.

away from shore. The source of wave-induced fluctuations in the air is shown to be located closer to shore, indicating that the transition from wave steepening to wave breaking is the precursor feature for the generation of wave-induced fluctuations in the airflow.

\subsection{Averaged profiles}

Time-averaged profiles were generated relative to the unrotated frame of reference ( $z$-axis parallel to gravity) to enable comparison with available studies. A reduced scale for water depth is introduced here too, $z / d$, incorporating the average water depth $(z)$ and the quiescent surface height $(d)$ at the local streamwise coordinate of $\eta$. The time-averaged velocity and r.m.s. profiles are first shown on the top row in figure 13 for the more offshore locations corresponding to the higher $\eta$ coordinates, and on the bottom row for the more onshore locations corresponding to the lower $\eta$ coordinates, respectively. The time-averaged wave-induced shear and normal fluctuations are similarly plotted in figure 14. Notwithstanding the differences in wave and bed slope, profiles of the averaged streamwise velocity in figure 13 may be qualitatively compared with the experimental data of Ting \& Kirby (1994, hereafter referred to as TK94) for 

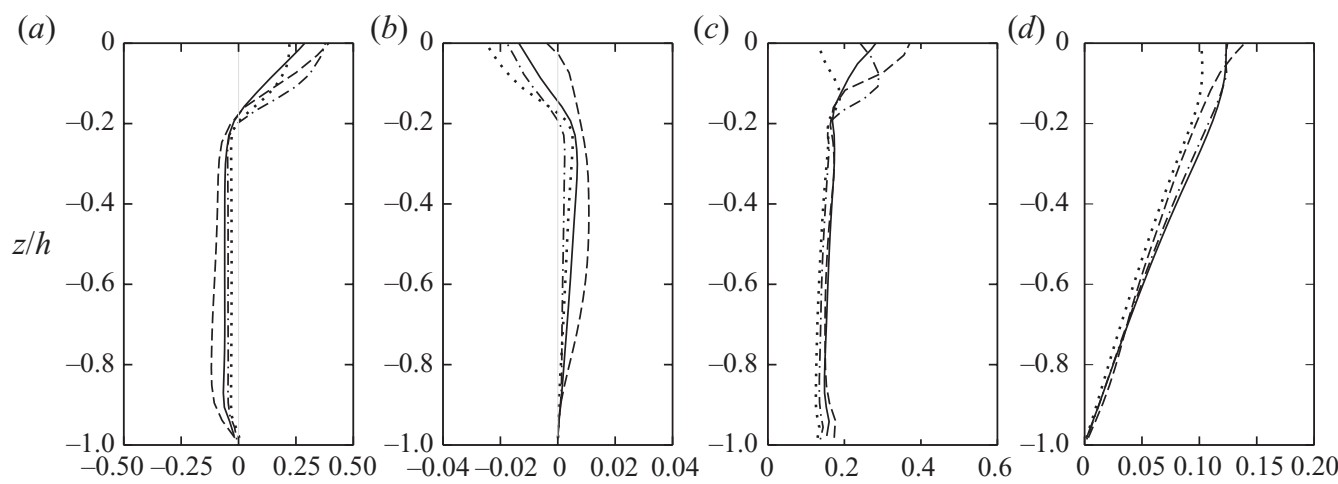

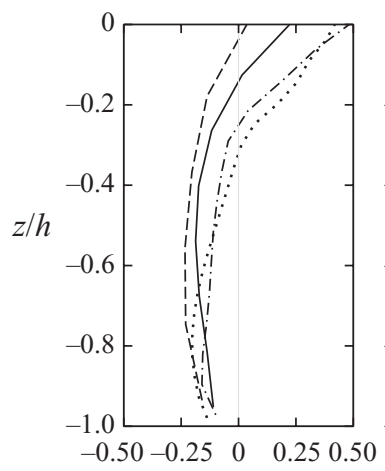

$\langle\bar{u}\rangle /(g d)^{1 / 2}$

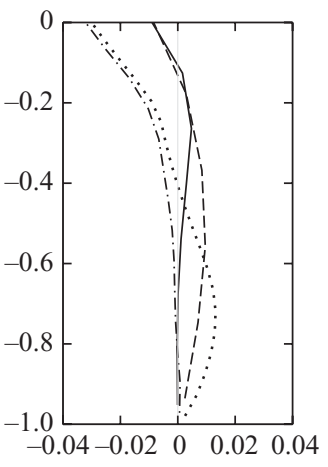

$\langle\bar{w}\rangle /(g d)^{1 / 2}$
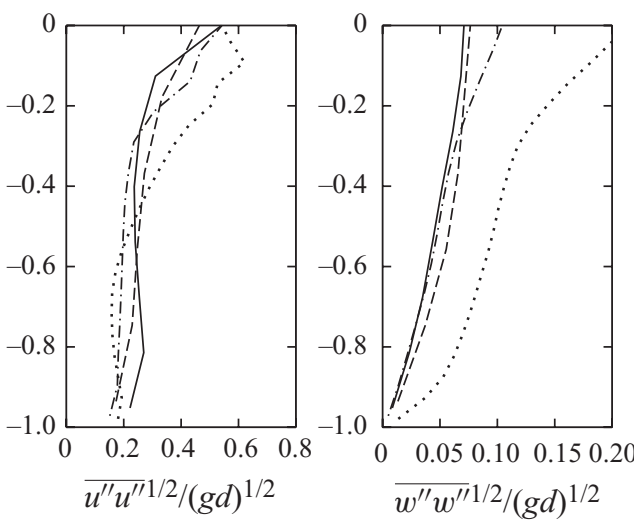

FIGURE 13. Time- and spanwise-averaged profiles of the average and r.m.s. $u$ - and $w$-velocities for varying $z$ (unrotated coordinates) (top): $\cdots, \eta=12.0 ; \cdots, \eta=10.4 ;-, \eta=8.8 ;--$, $\eta=7.3 ;$ (bottom): $\cdots, \eta=6.1 ; \cdot-\cdot ., \eta=4.2 ;-, \eta=2.3 ;--, \eta=1.7$.

spilling and plunging breakers, and to the simulation weak plunger results obtained by $\mathrm{CD}$. The figure helps to clearly identify the surface upper layer and sublayer zones defined in the Introduction. The surface sublayers are located within the narrow flow zone of $z / d \approx 0.2$. (Note that the definition $z / d$ compares with the $(z-\varepsilon) / h$ measure used in TK94, where $h$ is the depth relative to the time-mean free-surface level. Nearly identical for substantial stretches of $\eta$, the divergence of $h$ from $d$ is documented in TK94.)

The velocity profiles feature a relatively narrow region of shoreward liquid surface current driven by the shoreward wave propagation and breaking, and a broader seaward undertow region that serves to ensure volume conservation and hence no change in the time-averaged shore position. The profiles feature a combination of various shape characteristics from the spilling and plunging profiles documented in TK94. The shoreward profile in the surface upper layer from the current simulation seems to be online with the TK94 observation for plungers, but contradicts the fully seaward profiles seen at the most offshore locations reported in the TK94 spilling scenario. This qualitative comparison with the TK94 experiment indicates that the present flow is an intermediate scenario between the spilling and plunger cases, keeping in mind that the results may be biased by the lack of ergodicity of the data as discussed above. Near the breaking point, the depth of the sharp 

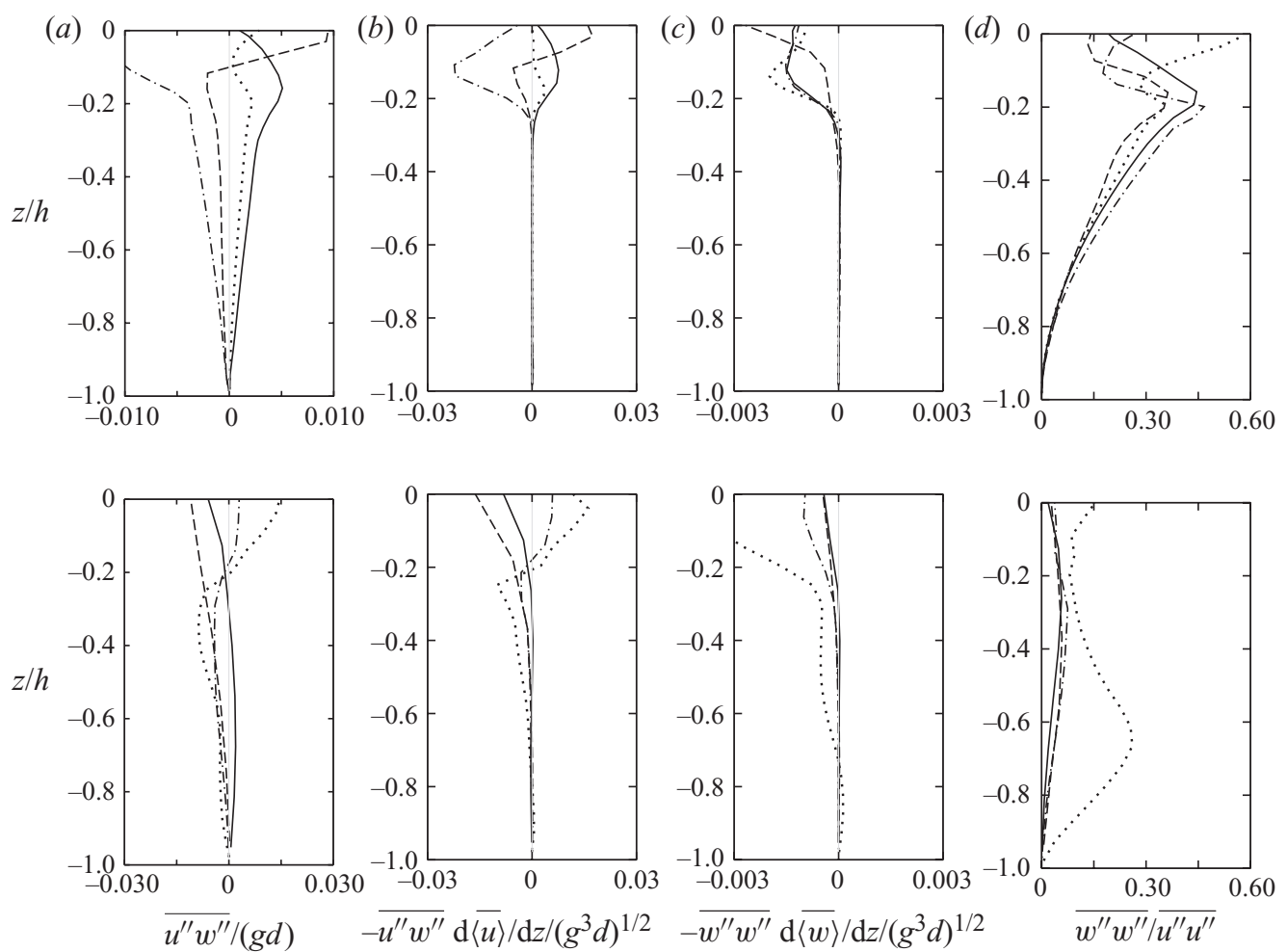

FIgURE 14. Time- and spanwise-averaged profiles of $(a)$ Reynolds shear stress, $(b)$ P13, (c) P33, (d) normal stress ratio, for varying $z$ (unrotated coordinates), at (top): $\cdots, \eta=12.0$; $. \cdot ., \eta=10.4 ;-, \eta=8.8 ;--, \eta=7.3$; (bottom): $\cdots, \eta=6.1 ; \cdot \cdot \cdot, \eta=4.2 ;-, \eta=2.3$; --, $\eta=1.7$.

transition point between the smooth undertow velocity profile and the high-shear onshore current at wave propagation level is $z / d \approx 0.23$, matching the TK94 results for plungers. This additional qualitative comparison supports the observation that waves produced in these simulations correspond more to energetic plungers than to spillers. Compared with the CD weak-plunger case, the strength of the shoreward current (as indicated by the magnitude of the velocity in the shoreward flow zone) is due to the higher wave amplitude. Regardless of the differences in problem set-up, the present profiles are qualitatively similar to the TK94 experiments and CD calculation results. Looking at the streamwise and vertical velocity profiles at locations upstream of the breaking point suggests that the average upper surface layer share some characteristics with a wall-jet flow as noted previously in the context of figure 11. The spanwise component $v$ is negligible everywhere until the breaking area $(\eta=7.3)$ is approached reflecting the onset of three-dimensionality and substantial wave-induced fluctuations.

The streamwise $\sqrt{\overline{u^{\prime \prime} u^{\prime \prime}}}$ fluctuation profiles depicted in the upper panels in figure 13 are shown to remain almost constant in the water core, and then to increase in magnitude as the surface is approached. Non-zero stress indicative of slight production at the seabed should be noted. One might expect a higher level of wave-induced fluctuations in proximity to the wall, decaying upward, but actually, however, there is 
a continuous seaward transport of these components by the mean flow from the surf zone, where it is created by breaking; this detail will be explained in the corresponding section. The trend in the bulk liquid flow well underneath the free-surface level is consistent with the observations reported in TK94. The magnitudes of wave-induced fluctuations in the simulation are higher than in the TK94 experiment, mainly because of the high $H_{0} / d$ ratio used in the present simulation. A notable difference between simulation and experiment in the fluctuating normal component $\sqrt{\overline{u^{\prime \prime} u^{\prime \prime}}}$ is the abrupt reduction to zero as the free surface is approached, as documented in TK94. This might actually be again another lack-of-ergodicity issue in our LEIS data and should as such be taken with care. Signal drop-out in the experiment and other differences in time-averaging in the interfacial zone makes any comparison of r.m.s. statistics in this region unreliable. Vertical stresses $\sqrt{\overline{w^{\prime \prime} w^{\prime \prime}}}$ are seen to increase moderately from the seabed to the surface sublayer, before changing as the surface is perceived above. It is perhaps important to note that unlike in unsheared fixed free -surface flows, $\sqrt{\overline{w^{\prime \prime} w^{\prime \prime}}}$ does not converge to zero as the free surface is approached from below. This is because the variance component treated here corresponds to the orbital wave component itself, reflecting its actual surface fluctuations. The spanwise wave-induced stresses $\sqrt{\overline{v^{\prime \prime} v^{\prime \prime}}}$ are negligible until the breaking point. As far as the shear stresses are concerned (shown in figure $14 a$ ), the $\overline{u^{\prime \prime} w^{\prime \prime}}$ component is dominant, increasing linearly in magnitude from the seabed to the surface sublayer.

At the other locations closer to shore (lower panels in figures 13 and 14), the same tendency is repeated for the wave-induced stresses. The streamwise component is the main contributor to the wave kinetic energy, while the component $\overline{u^{\prime \prime} w^{\prime \prime}}$ dominates the wave-induced shear everywhere in the flow, and in the surface upper layer and sublayer in particular. The largest $\overline{u^{\prime \prime} w^{\prime \prime}}$ shear component occurs as expected at the breaking point and in the area of plunger impact on the free surface. Figure $14(d)$, which displays the ratio of streamwise and vertical stresses, reveals that $\sqrt{\overline{w^{\prime \prime} w^{\prime \prime}}}$ is important in comparison with the streamwise component in the surface sublayer and generally decays rapidly downwards as the seabed surface is approached, in accordance with the TK96 data. This is because $\sqrt{\overline{w^{\prime \prime} w^{\prime \prime}}}$ decays with distance from the surface, while $\sqrt{\overline{u^{\prime \prime} u^{\prime \prime}}}$ varies less uniformly across depth. The ratio varies abruptly between 0.2 and 0.5 across the surface sublayer in the breaking and surf zones in particular, but on average it remains smaller compared with that found in the proximity of solid walls, and in plane jets and wakes. The present results are in complete agreement with the trends documented in TK96 for plunging breakers, and contrast with the trends reported in TK96 for spilling breakers. The behaviour at $\eta=6.1$ coincides with the area of plunger impact, given the downward penetration of the plunger through the surface sublayer.

Looking carefully at the present LEIS results of the variance distributions in the liquid side suggests very strong similarities also with the DNS results of wind-wave generation processes of Lin et al. (2008). In examining the transient (non-timeaveraged as in the present work) effects of waves on turbulence statistics below the interface, they found that the wave effect on the turbulent velocity variances is substantial in the water. Although the flow treated in their work is different from the present set-up, the turbulent velocity variance distributions below the surface share various properties: (i) turbulence is strongly anisotropic as the interface is approached and (ii) a clear dominance is found of the streamwise velocity variance component over the other terms, with a rate of turbulence anisotropy $\sqrt{\overline{u^{\prime \prime} u^{\prime \prime}}} / \sqrt{\overline{w^{\prime \prime} w^{\prime \prime}}} \sim 2.5-5$. In 
our simulations though, this rate attains one order of magnitude in the outer- and inner surf zones.

\subsection{Energy exchange mechanisms}

The other key quantity to be quantified based on averaged profiles is the rate of energy exchange between the mean flow and the modal scales (wave plus subscale turbulence). For that purpose, we proceed by defining the kinetic energy of the mean

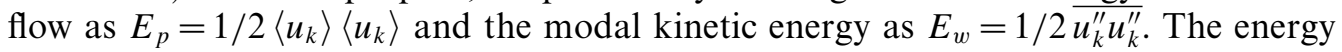
exchange term between the mean and the wave modes therefore becomes

$$
P_{i j}=-\overline{u_{i}^{\prime \prime} u_{j}^{\prime \prime}} \frac{\partial \overline{\left\langle u_{i}\right\rangle}}{\partial x_{j}},
$$

with the most significant components being $P_{13}$ and $P_{33}$. Energy is transferred from the mean to the wave modes when these terms are positive and vice versa. The exchange terms $P_{13}$ and $P_{33}$ are shown in figure $14(b, c)$. The streamwise and vertical velocity gradients normal to the interface in the surface sublayer, together with the wave-induced shear and normal stresses, suggest that the energy exchange terms are particularly active in this region. The transfer between the mean and the wave modes operates both ways, forward and backscatter, in the tiny region $z / d \approx 0.2$, below which the vertical variations in both velocities $\langle u\rangle$ and $\langle w\rangle$ are negligible. However, since the horizontal velocity gradient is positive in the surface sublayer, the direction of energy flow is controlled by the sign of $-\overline{u^{\prime \prime} w^{\prime \prime}}$. The transfer component $P_{13}=-\overline{u^{\prime \prime} w^{\prime \prime}} \overline{\partial\langle u\rangle} / \partial z$ is generally positive in the early phase of wave build-up and breakup, which is indicative of energy transfer from the mean to the wave modes. The backscatter of energy from the wave modes to the mean operates mainly near the breaking point and immediately shoreward of it. In all locations, the transfer by $P_{33}=-\overline{w^{\prime \prime} w^{\prime \prime}} \partial \overline{\langle w\rangle} / \partial z$ is directed one way - from the mean to the waves, due to the $w$-velocity gradient being inverted at some point in depth, reflecting the penetration of the waves downwards. The vigour of this component results from overturning of the crest and penetration of the jet into the water.

In the contribution of Lin et al. (2008), the same mechanisms of energy transfer from wind to waves have also been dealt with, but in a different way as the purpose was to understand wave growth processes. Their study showed that energy transfer mechanisms originate from turbulence-induced pressure fluctuations at the surface in the linear growth stage and are due to the in-phase relationship between wave slope and wave-induced pressure fluctuations in the exponential growth stage.

\section{Zonal conditional analysis}

\subsection{The flow regime map}

Visual assessment of wave-breaking events suggests a plausible delineation of the flow map into regions featuring different topology characteristics, somewhat in conformity with the decomposition proposed by Christensen et al. (2002). Figure 15 shows the delineation of zones adopted in the current study: each zone features specific transport processes taking place repeatedly at various instants, while the nature of the transport processes may differ significantly between zones. The motivations for such an analysis have already been alluded to when discussing the longshore time-averaged quantities. Unlike in most single-phase flows amenable to LES, the present context requires the 

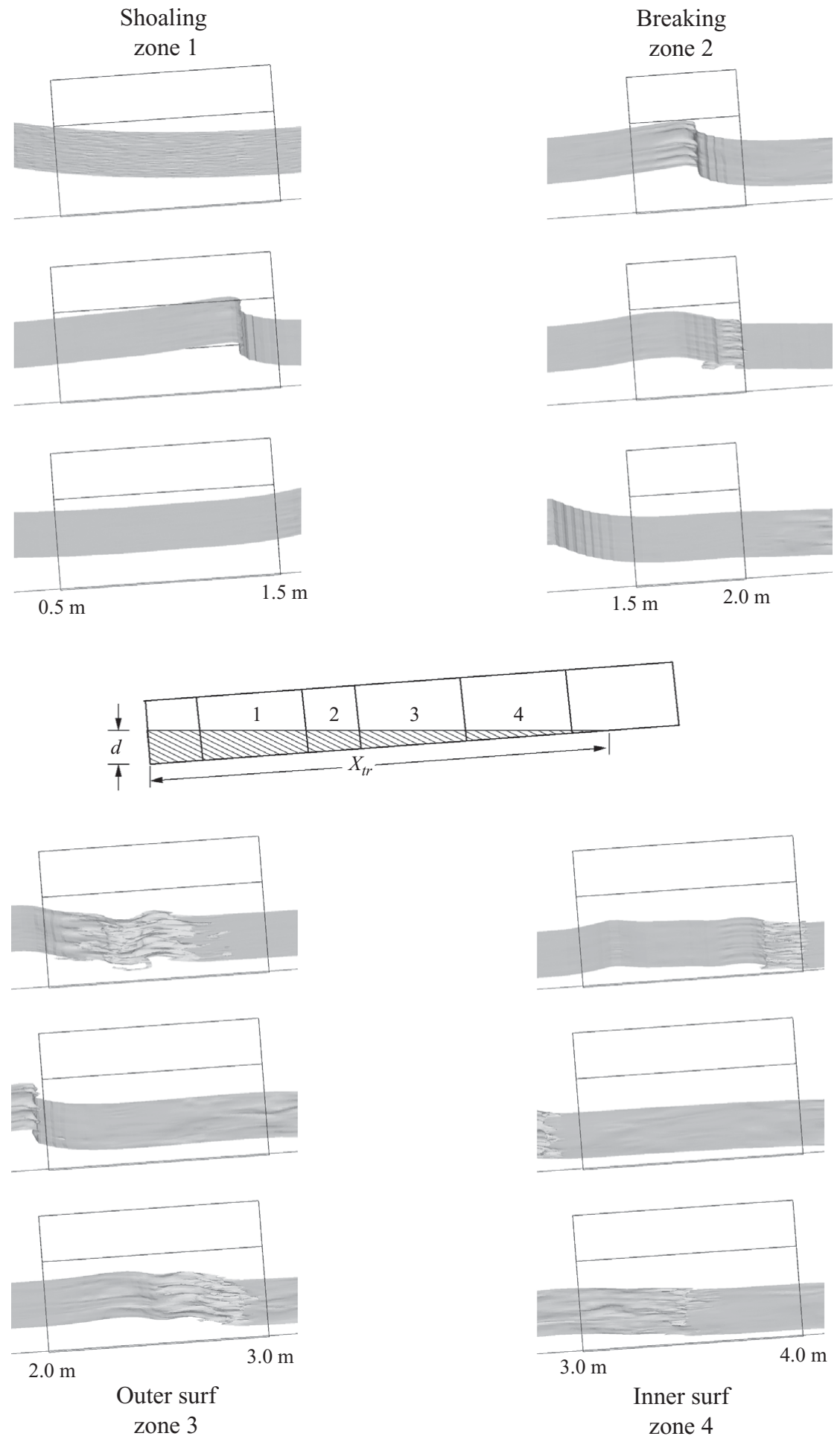

FIGURE 15. Delineation of zones in the wave flow, according to the wave action that predominates in each zone. To the right of zone 4 is the area commonly referred to as the 'swash' zone; this zone is not an area of interest in the current work. 
identification of specific events, not necessarily periodic or homogeneous as would be required, for example, in triple flow decomposition applied to coherent-structuredominated flows. This is true for most multiphase flow systems, and the present one in particular.

Zone 1 is the shoaling/deformation zone furthest offshore, located at $8.9 \geqslant \eta \geqslant 12.0$ from the quiescent shoreline location. This zone is characterized by low waveslope and high Froude number. Zone 2 is the main breaking zone lying in the interval $7.3 \geqslant \eta \geqslant 8.9$ from the shoreline and is characterized by high wave-slope and maximal wave-crest height. The Froude number here is slightly smaller than that in zone 1 . Zone 3 is the outer surf zone located in the region $4.2 \geqslant \eta \geqslant 7.3$ from the shoreline and is characterized by the post-breaking aftermath. Zone 4 is the inner surf zone located at $1.1 \geqslant \eta \geqslant 4.2$ from the shoreline and is adjacent to the swash zone (which includes the shoreline), a flow area characterized by a low Froude number. The analysis is focused here on each of these zones separately. The swash zone and, to a lesser extent, the shoaling zone are not of particular interest in the current work. The analysis presented in the next section deals with the most pertinent flow features associated with breaking, including small-scale breaking, kinetic energy production and redistribution, dissipation and enstrophy transport.

\subsection{Interfacial area evolution and small-scale breaking}

A notable feature of the breaking events shown in figure 9 is the formation of small-scale surface deformations perpendicular to the spanwise direction, wrinkling in the longshore direction. This process may be referred to as surface 'small-scale breaking' - to contrast with 'micro-breaking' which is characterized by a bore-like crest accompanied by parasitic capillary waves riding along the forward face (Banner \& Phillips 1974) - when this is uncorrelated with the dominant 2D wave propagation and breaking. Small-scale breaking is the first visible evidence of three-dimensionality in flows driven by nominally 2D waves. Jaggedness of the wave crest and curvature in the alongshore direction are ubiquitous features of breaking waves. Physical models featuring simplistic representations of interface kinematics - including 2D simulations - cannot capture 'small-scale breaking', the causes of which and, more particularly, its implication for turbulent processes in the flow are explored next.

A modified version of the measure introduced by Geurts (2002) is used here to quantify surface wrinkling identified as the precursor to 'small-scale breaking'. Expressed in a non-dimensional manner, it reads

$$
W=\frac{L_{0}+2 H_{0}}{L_{0} H_{0}} \frac{\int_{S}|\kappa| \mathrm{d} S}{\int_{S f l a t} \mathrm{~d} S},
$$

where $\int_{S \text { flat }} \mathrm{d} S$ is the interfacial area of a smooth free surface parallel to the seabed (denoted by $A_{0}$ ), and is introduced to make the wrinkling measure independent of quadrant size. The parameter $\left(L_{0}+2 H_{0}\right) /\left(L_{0} H_{0}\right)$ is introduced to scale the wrinkling measure with the curvature of the underlying $2 \mathrm{D}$ wave; it is equal to 9.24 in this flow. Two measures of wrinkling are generated: (i) $W^{3 D}$, based on the free surface of the $3 \mathrm{D}$ flow and (ii) $W^{2 D}$, based on the free-surface locus of the spanwise-averaged flow field. If $W^{2 D}$ is interpreted as the wrinkling associated with the underlying $2 \mathrm{D}$ 
wave, then the difference between $W^{3 D}$ and $W^{2 D}$ emphasizes the existence of surface deformations that may be classified as 'small-scale breaking'. Finally, the interfacial area $A$ scaled with the flat surface $A_{0}$ has also been determined for comparison with $W$ :

$$
A=\frac{\int_{S} \mathrm{~d} S}{\int_{S f l a t} \mathrm{~d} S} .
$$

While wrinkling measures $W^{3 D}$ and $W^{2 D}$ are specifically dedicated to measuring small-scale wrinkling, the interfacial area $A$ contains both small-scale and largescale surface deformations, and as such it does not delineate 3D surface 'smallscale breaking' from 3D wave deformation. Surface deformations falling within this category are such that the normalized interfacial area $A / A_{0}$ is somewhat larger than unity, but this is not clear yet. Figure 16, which illustrates the time evolution of $W$ and $A / A_{0}$, helps distinguish nearshore from offshore flow zones. Periodicity is seen to clearly prevail in zone 1 only, the area populated by the low-frequency wave-wave interaction mentioned in the Introduction.

Figure 16(a), which compares 2D and 3D wrinkling, shows that the free surface in zone 1 generally features low curvature, with occasional crests in the wrinkling signal coinciding with wave-crest steepening as zone 2 is approached. There is little difference between 2D and 3D wrinkling signals. (The appearance of 'small-scale breaking' wrinkles at the start is due to the white-noise perturbation imposed on the initial flow field.) Negligible wrinkling generally prevails in zone 2, with the exception of the breaking event during which surface deformations are systematically accompanied by 3D 'small-scale breaking'. These occasional surface deformations observed in this zone coincide with the immediate pre-breaking event when the wave saturates before plunging. Peak values in the wrinkling measure for zone 2 correspond to the type of free-surface configuration shown in the first panel of zone 2 in figure 15. According to the signals taken in this zone, 2D wrinkling dominates the overall surface deformations and is associated with the jet being thrown forward of the crest during wave breaking. During this event, air bubbles are trapped in the jet and entrained underneath the free surface. 'Small-scale breaking' contribution during wave plunging in this zone is seen to take the form of a longshore instability in the jet.

Following start-up, zone 3 in figure 16 shows sustained and significant highfrequency topology deformations. The event resulting in the most concentrated interface wrinkling coincides with the collision of backwash water from the inner surf zone 4, with the jet and splash water thrown towards shore. The top free-surface snapshots in zone 3 in the figure shows a rib-like surface deformation of the same form as identified by Watanabe et al. (2005) for plunging breakers. The time signal shows $W^{2 D}$ at maximum wrinkling to be relatively insignificant, suggesting that wrinkling is actually dominated by 'small-scale breaking'. Subsequent troughs in the $W^{3 D}$ signal show that interface smoothness in zone 3 is periodically regained between periods of significant wrinkling, as shown in zone 3 snapshots of figure 15 . The backwash of water can again be seen to contribute significantly to 'small-scale breaking' in zone 4; the wrinkles actually propagate into zone 4 from the outer surf zone 3. Beyond a superficial description of wrinkling in the wave-breaking flow, macro-measures such as $W^{3 D}, W^{2 D}$ and $A$ at this stage do not completely answer open questions regarding 
Zone 1
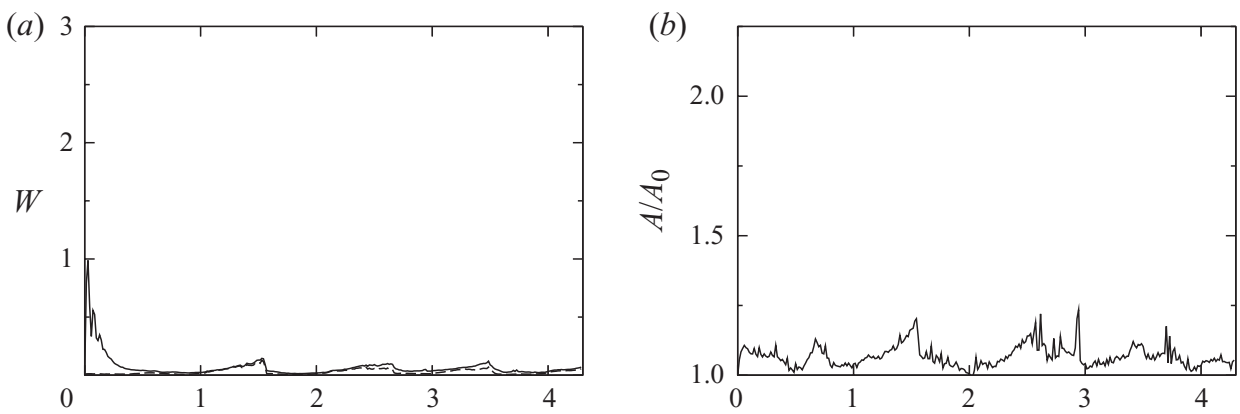

Zone 2
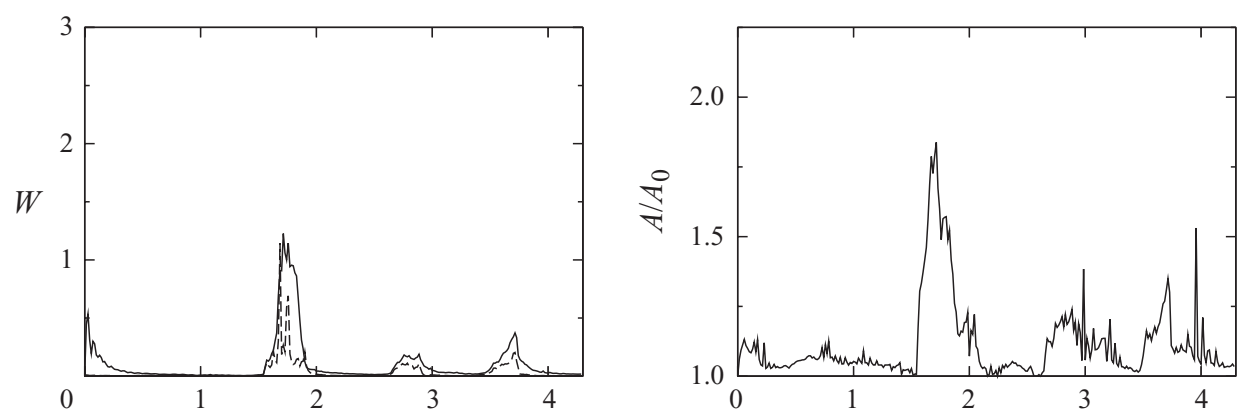

Zone 3
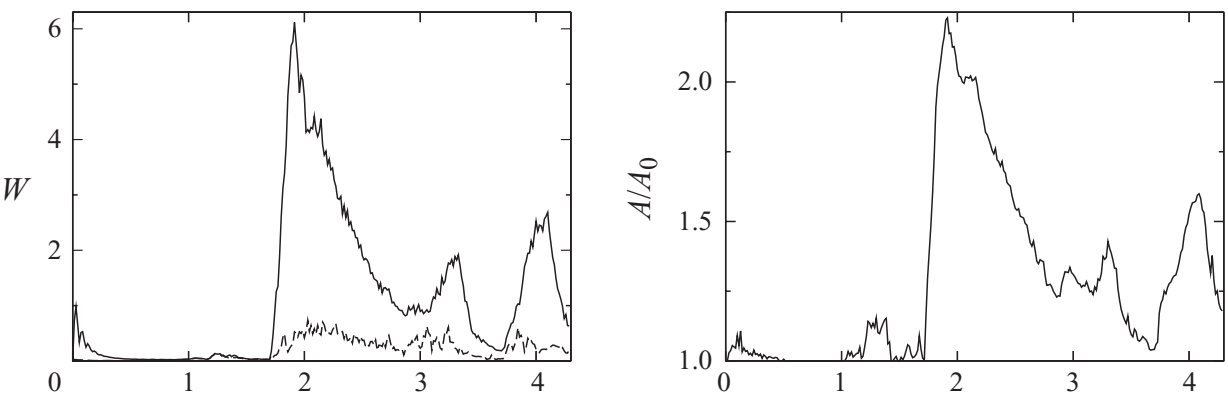

Zone 4
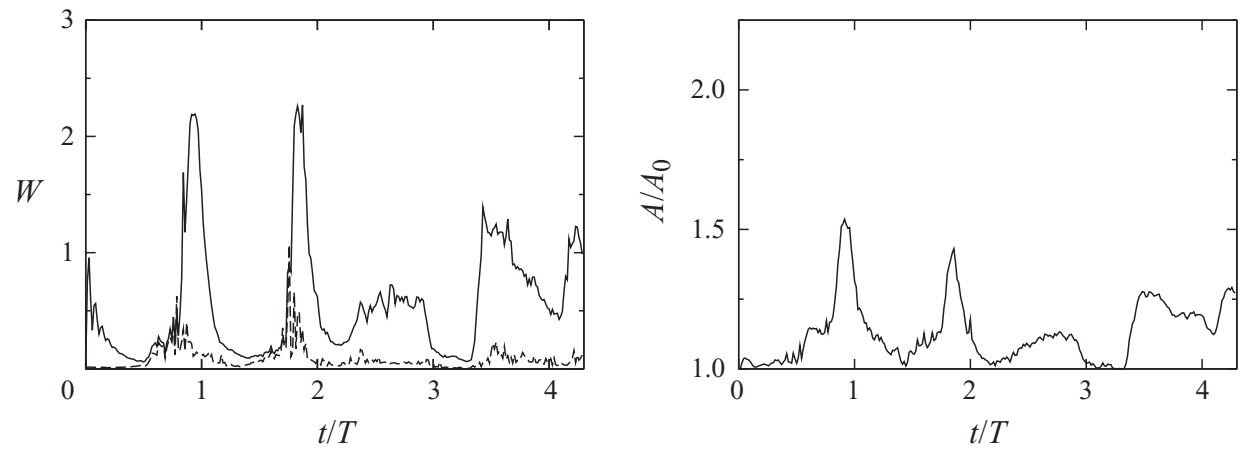

FIGURE 16. Time histories of the wrinkling $(a)$ and interfacial area $(b)$. For the wrinkling, the solid line is the signal for the $3 \mathrm{D}$ wrinkling, while the dashed line is the $2 \mathrm{D}$ wrinkling of the spanwise-averaged free surface. 
interface wrinkling, e.g. the manner in which 'small-scale breaking' occurs in the backwash surf zones.

\subsection{Turbulent transport processes}

\subsubsection{Definitions}

Complementing $\S 8$, in which the exchange between the mean and the modes (encompassing wave and small-scale fluctuations) has been analysed, this section addresses the mode-mode interaction mechanisms, the exchange between coherent (in terms of time scale) large-scale waves and non-coherent small-scale turbulence. The flow field and interface location generated by spanwise-averaging are interpreted as the organized motion associated with $2 \mathrm{D}$ wave; the deviation from this average is considered to constitute the turbulent fluctuating field. This approach is proper for the treatment of temporally evolving mixing layers, and has also been shown to be useful for wave-breaking experiments, e.g. Ting \& Kirby (1996). The flow analysis is therefore based solely on spanwise-averaging, i.e. $\varphi=\langle\varphi\rangle+\varphi^{\prime}$, where $\langle\cdot\rangle$ represents the spanwise-average and $\varphi^{\prime}$ is the fluctuating component over this average. In the following analysis, TKE will, for simplicity, be denoted by $K$.

The TKE budget analysis is restricted here to the liquid phase and is based on conventional forms used in LES and DNS studies of turbulent single-phase flow. On the basis of the approaches of Calhoun \& Street (2001) and Reboux et al. (2006), the resolved TKE equation is expressed here as

$$
\frac{\mathrm{D} K}{\mathrm{D} t}=P+T+D_{p}-\varepsilon+v \nabla^{2} K
$$

where

$$
\begin{aligned}
\text { production: } \quad P & =-\left\langle u_{i}^{\prime} u_{j}^{\prime}\right\rangle \frac{\partial\left\langle u_{i}\right\rangle}{\partial x_{j}}, \\
\text { turbulent + SGS transport: } \quad T & =-\frac{\partial}{\partial x_{j}}\left(\left\langle u_{i}^{\prime} u_{i}^{\prime} u_{j}^{\prime}\right\rangle+\left\langle u_{i}^{\prime} \tau_{i j}\right\rangle\right), \\
\text { pressure diffusion: } \quad D_{p} & =-\frac{1}{\rho} \frac{\partial}{\partial x_{i}}\left\langle p^{\prime} u_{i}^{\prime}\right\rangle, \\
\text { viscous + SGS dissipation: } \quad \varepsilon & =v\left\langle\frac{\partial u_{i}^{\prime}}{\partial x_{j}} \frac{\partial u_{i}^{\prime}}{\partial x_{j}}\right\rangle-\left\langle\frac{\partial u_{i}^{\prime}}{\partial x_{j}} \tau_{i j}\right\rangle .
\end{aligned}
$$

(Note the reversion from double prime $\varphi^{\prime \prime}$ back to single prime $\varphi^{\prime}$, since we are no longer time-averaging.)

Conditional zonal averaging is used here to identify the zones where turbulence is generated and interact with the $2 \mathrm{D}$ wave motion. Conditional averages are defined here according to a specific condition $k$, referring to point $\boldsymbol{x}$ being located within a specific subdomain (zone $k=1-4$ ) in a specific phase (air or liquid). For this purpose, we define a conditionally averaged quantity $\left.\langle\varphi\rangle\right|_{k} ^{h}$ representing the volumeaverage over conditions $k$ and $h$, denoting the integration volume within zones 1-4 $\left(k=V_{k} ; k=1,4\right)$, for a specific fluid phase $(h=L, G)$. The colour function distribution criterion is used for the purpose: $\langle C\rangle=0$ for liquid, $\langle C\rangle=1$ for gas, and mixed-phase cells are ignored so as not to pollute essentially single-phase analyses. The conditional, 
zonal-averaged phase-specific TKE is defined as

$$
\left.\langle T K E\rangle\right|_{k} ^{h}=\frac{1}{f_{k}} \frac{\int_{\left.V\right|_{k} ^{h}} K \mathrm{~d} V}{\int_{\left.V\right|_{k} ^{h}} \mathrm{~d} V} .
$$

Similarly, the conditional, zonal-averaged TKE budget component (or residual) is defined as

$$
\left.\langle T K E-b\rangle\right|_{k} ^{h}=\frac{1}{f_{k}^{\prime}} \frac{\left.\int_{\left.V\right|_{k} ^{h}} \frac{D K}{D t}\right|^{b} \mathrm{~d} V}{\int_{\left.V\right|_{k} ^{h}} \mathrm{~d} V} .
$$

A conditional, zonal-averaged, phase-specific enstrophy is determined in the same way as a measure of turbulence decay or dissipation:

$$
\left.\left\langle\omega^{2}\right\rangle\right|_{k} ^{h}=\frac{1}{f_{\omega^{2}}} \frac{\int_{\left.V\right|_{k} ^{h}} \omega^{2} \mathrm{~d} V}{\int_{\left.V\right|_{k} ^{h}} \mathrm{~d} V} .
$$

The scaling parameters introduced here use the shear velocities and the maximum bath depth as the characteristic velocity and length scales, respectively. As used in the form drag computation, the averaged interfacial shear velocity from the airside is taken to be $U_{\operatorname{IntG}}^{\tau}=0.019 \mathrm{~m} \mathrm{~s}^{-1}$, while the water-side value is taken to be $U_{\text {Int } L}^{\tau}=0.0013 \mathrm{~m} \mathrm{~s}^{-1}$. Zonal-averaged TKE and enstrophy signals are generated for the entire flow domain. To analyse the results for different phases, a single scaling factor is used in each case, based on the air-side $U_{I n t G}^{\tau}$ value:

$$
f_{k}=\frac{U_{I n t G}^{\tau}{ }^{3} d_{0}}{v_{G}} \approx 0.147, \quad f_{\omega^{2}}=\frac{U_{I n t G}^{\tau} \sqrt{g d}}{v_{G} d_{0}} \approx 133 .
$$

Because the TKE budget is performed only on the water flow, $U_{\text {Int } L}^{\tau}$ is used for scaling, i.e.

$$
f_{k^{\prime}}=\frac{U_{I n t L}^{\tau} \sqrt[3]{g d_{0}}}{v_{G}} \approx 0.0039
$$

\subsubsection{TKE and enstrophy time signals}

Enstrophy (a global measure of the vorticity content of a flow and the square of its $L^{2}$ norm) is specifically introduced here to measure the intensity of kinetic energy induced by large-scale vortical motions. This is done here to distinguish enstrophy from TKE reflecting the intensity of the small-scale fluctuating field superposed on the 2D wave. Figure $17(a, b)$ presents the TKE and conditionally averaged enstrophy signals, respectively. White-noise perturbations imposed on the initial field are reflected in the initial phase of the signals. Liquid shoaling (zone 1) and breaking (zone 2) are clearly shown to be low-turbulence regions, while the inner and outer (zones 3 and 4) are high-turbulence, 3D flow regions, with TKE values attaining an order of magnitude higher.

Airflow TKE signals $\left.\langle T K E\rangle\right|_{k} ^{G}$ feature significant differences from the water-side over substantial durations of the wave lifetime. The intensity of the signal is generally 
Zone 1
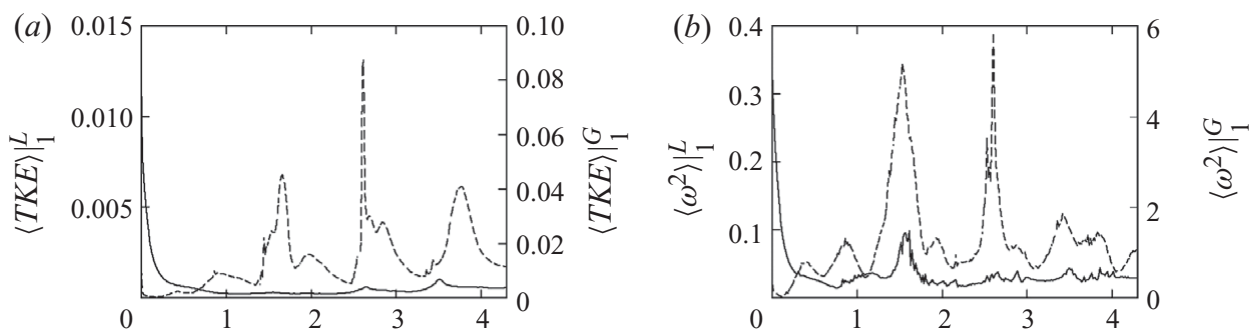

Zone 2
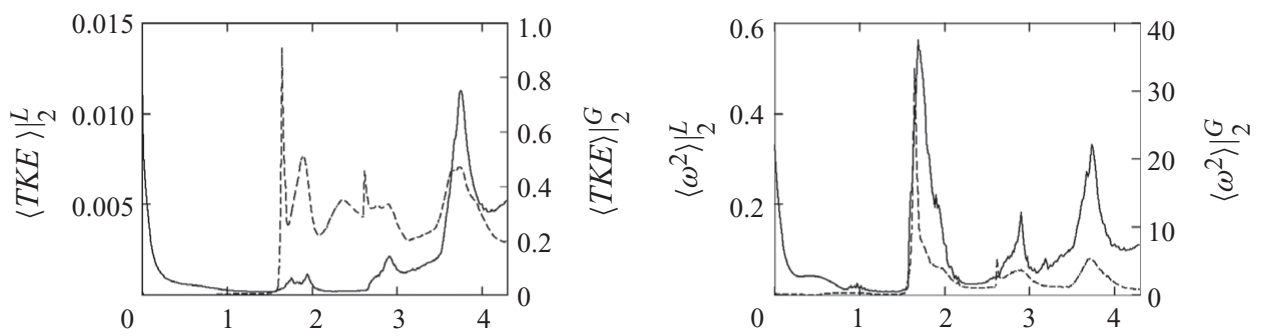

Zone 3
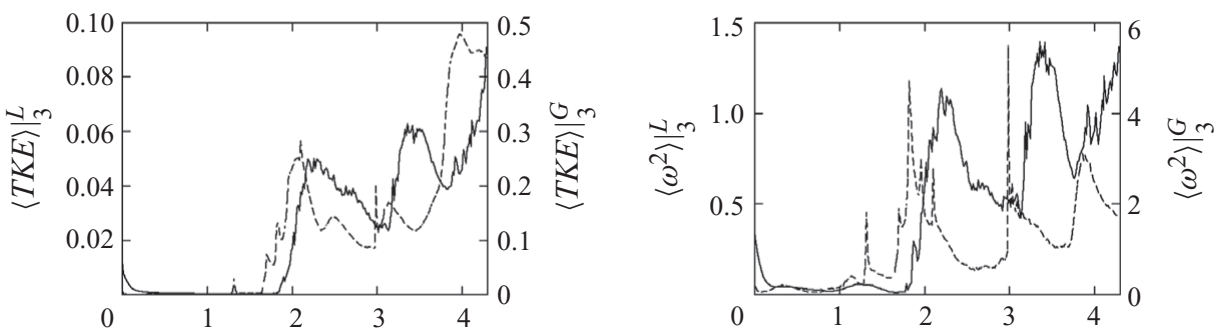

Zone 4
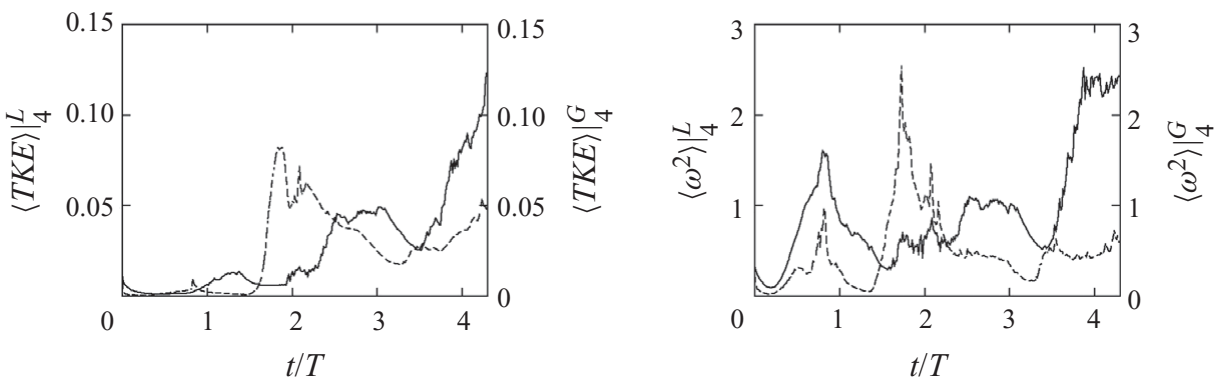

FIGURE 17. Time histories of zone-averaged quantities: $(a)$ TKE and $(b)$ enstrophy. The liquid-sided signal is denoted by solid line, while the gas-sided signal is denoted by the dashed line.

higher than in the liquid except at the outermost backwash zone where the flow is dominated by whitecaps populated by 'small-scale breaking' structures. While the airflow TKE tends to subside relatively rapidly, it tends to build up in the liquid after each wave-breaking event. Accumulations of TKE in zones 3 and 4 imply that the flow has not yet reached ergodic conditions; higher-order statistics require longer 
Zone 1

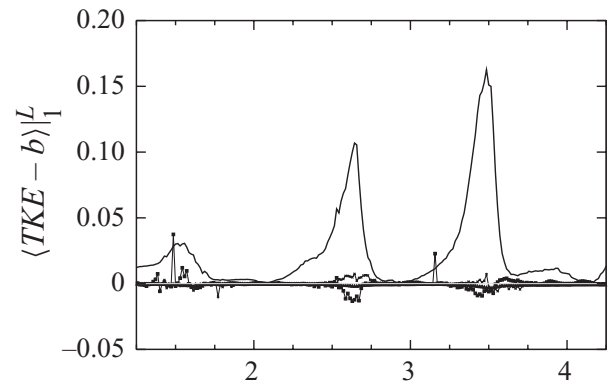

Zone 3

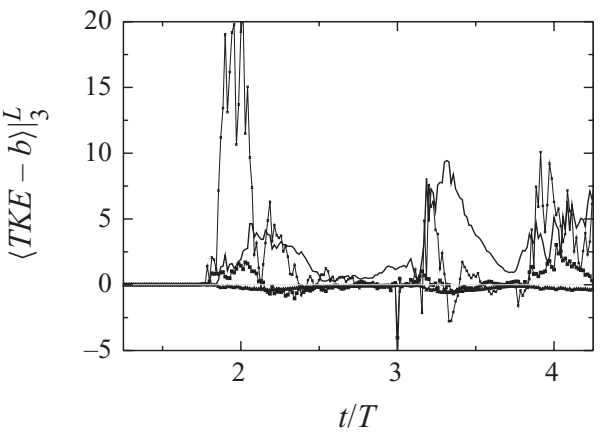

Zone 2

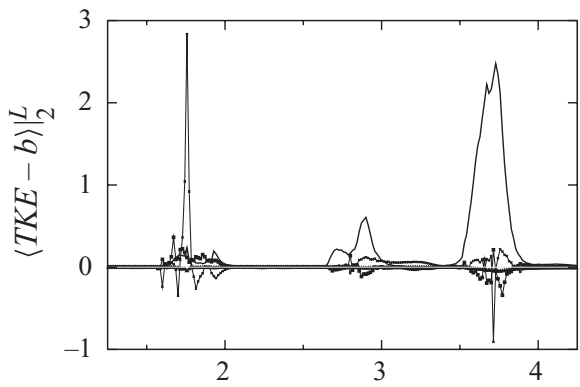

Zone 4

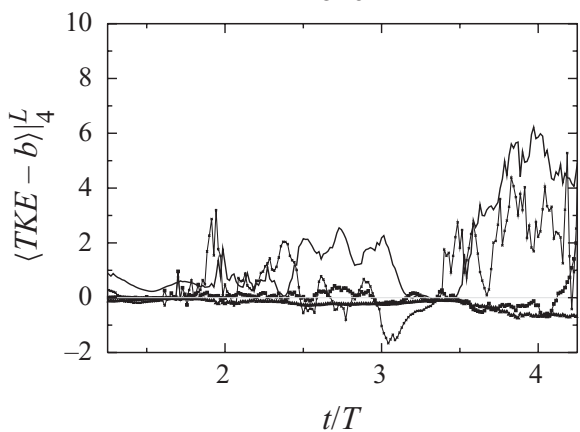

FIGURE 18. Time histories of zone-averaged terms in the liquid-sided TKE budget: production; $\longrightarrow$, transport; $\boldsymbol{A}$, dissipation; $\rightarrow$, diffusion.

simulations, but this is not the scope of the present investigation. During wave buildup and propagation within zones 1 and $2,\langle T K E\rangle>\left.\right|_{1,2} ^{G}$ and $\left.\langle T K E\rangle\right|_{1,2} ^{L}$ are generally not highly correlated. Signals in the inner and outer surf zones 3 and 4 are visibly correlated but appear $180^{\circ}$ out of phase. This shift in phase occurs because the generation of air-side small-scale TKE is antecedent to the liquid. This will be discussed with coherent structures evolution in the corresponding section.

Turning now to the enstrophy signals, the energy induced by the vorticity dynamics is shown to be stronger in the air than in the water, at least for the first two outermost offshore zones. As long as the flow is nominally two-dimensional, $\left.\left\langle\omega^{2}\right\rangle\right|_{1,2} ^{G}$ and $\left.\left\langle\omega^{2}\right\rangle\right|_{1,2} ^{L}$ signals seem to be in phase, suggesting that vorticity structures above the sea surface are somewhat correlated with the structures in the liquid beneath it. Just as for the TKE signals in zones 3 and 4 , air and liquid enstrophy signals are $180^{\circ}$. out of phase. Strong rises in the liquid-phase TKE signals $\left.\langle T K E\rangle\right|_{3,4} ^{L}$ are accompanied by strong rises in the enstrophy in the $3 \mathrm{D}$ inner and outer surf zones.

\subsubsection{TKE budget time signals}

Time series of individual $\left.\langle T K E-b\rangle\right|_{k} ^{h}$ contributions are plotted in figure 18; time variation and advection are not considered. A close inspection of inner and outer surf-zone signals reveals that during the simulated period wave, breaking is characterized by high sustained levels of turbulence production, pressure diffusion and transport, and lower levels of dissipation. Viscous diffusion and dissipation are negligible compared with the modelled SGS parts, which is the main reason for summing them up here. Net contributions of pressure diffusion and transport in zones 1 and 2 only occur during wave build-up. Comparing figures 17 and 18, drastic 
TKE growth in zones 3 and 4 takes place initially as a result of turbulent and SGS transport, and only later on by shear-induced production. Pressure diffusion and SGS dissipation in the inner and outer surf zones gain in importance only at the later stage of the simulation, where they tend to balance the production and turbulence transport, but that is not yet accomplished while the rate of change of TKE remains positive. TKE convection by the mean flow $\partial\left(u_{j} K\right) / \partial x_{j}$ is sustained by the action of the wave generator and contributes in entraining air while resisting buoyancy forces. Lamarre \& Melville (1991) observed that up to $50 \%$ of wave energy (both mean and turbulent) is spent in entraining air and resisting buoyancy forces. It is perhaps important to note that the present LEIS cannot resolve features of the inner and outer surf zone flows such as the foamy mixed layer formed by ocean spray.

\subsection{Longshore turbulent transport processes}

\subsubsection{TKE longshore evolution}

Zone-specific time signals discussed previously are complemented here by contours of the spanwise-averaged distribution of TKE in figure 19. The contour plots are for two periods. The outer surf zone 3 features the first major jumps in TKE level due to the impact of the plunging breaker on the free surface. The subsequent rise in TKE in the inner surf zone 4 is representative of the landward transport of TKE noted by Ting \& Kirby (1994) for plunging waves. More pointedly, high TKE levels in zone 4 suggest that the inner surf zone receives turbulence from zone 3 and produces turbulence by backwash. Additional contributions to three-dimensionality may arise from longshore free-surface instabilities associated with swash. There is also a noticeable spread of the high-TKE zone seaward into breaking zone 2; whether this spread continues over time was not ascertained in the current study. As a weak plunger, however, the current flow expectedly features characteristics resembling those of spilling breakers, such as the seaward transport of TKE. A noteworthy feature apparent in figure 19 is the sign of the vertical gradient of TKE in shoaling (zone 1) and breaking (zone 2): $\partial K / \partial z$ is positive initially before inverting sign in the very thin surface sublayer. Therefore, in effect a 'TKE point source' is formed in the core of the rolling wave before breaking. This feature is important for the diffusion of kinetic energy down to the core water flow (Svendsen 1987), as discussed next.

In summary, TKE generation is shown to be a continuous process during which turbulence is introduced by each subsequent breaking, an observation that is at odds with the assertion of Christensen et al. (2002), according to whom turbulence 'dies out' between successive plungers. TKE is first generated at the wave crest in zone 2 due to interfacial shear, after which it is transported all the way down by the waveplunging motion. This motion creates further turbulence as it impacts on the outer surf zone. Part of the TKE at impact in zone 3 is spread landward towards zone 4, then washed back seawards by the mean flow. Understanding this cyclic mechanism is very important for sediment transport in particular, which represents an issue of great interest at present. According to figure 18, the dissipation of kinetic energy by viscous and turbulent effects is a rather slow process. This can be judged from the contours in figure 19: after four wave periods, the TKE transported seawards still has not traversed more than $15 \%$ of the entire domain.

\subsubsection{TKE longshore budget}

Figure 20 shows spatial distributions of the production, turbulent transport and pressure diffusion contributions in the conditional zonal-averaged TKE budget. Being negligibly small, dissipation terms are not discussed. In relation to the local intensity 

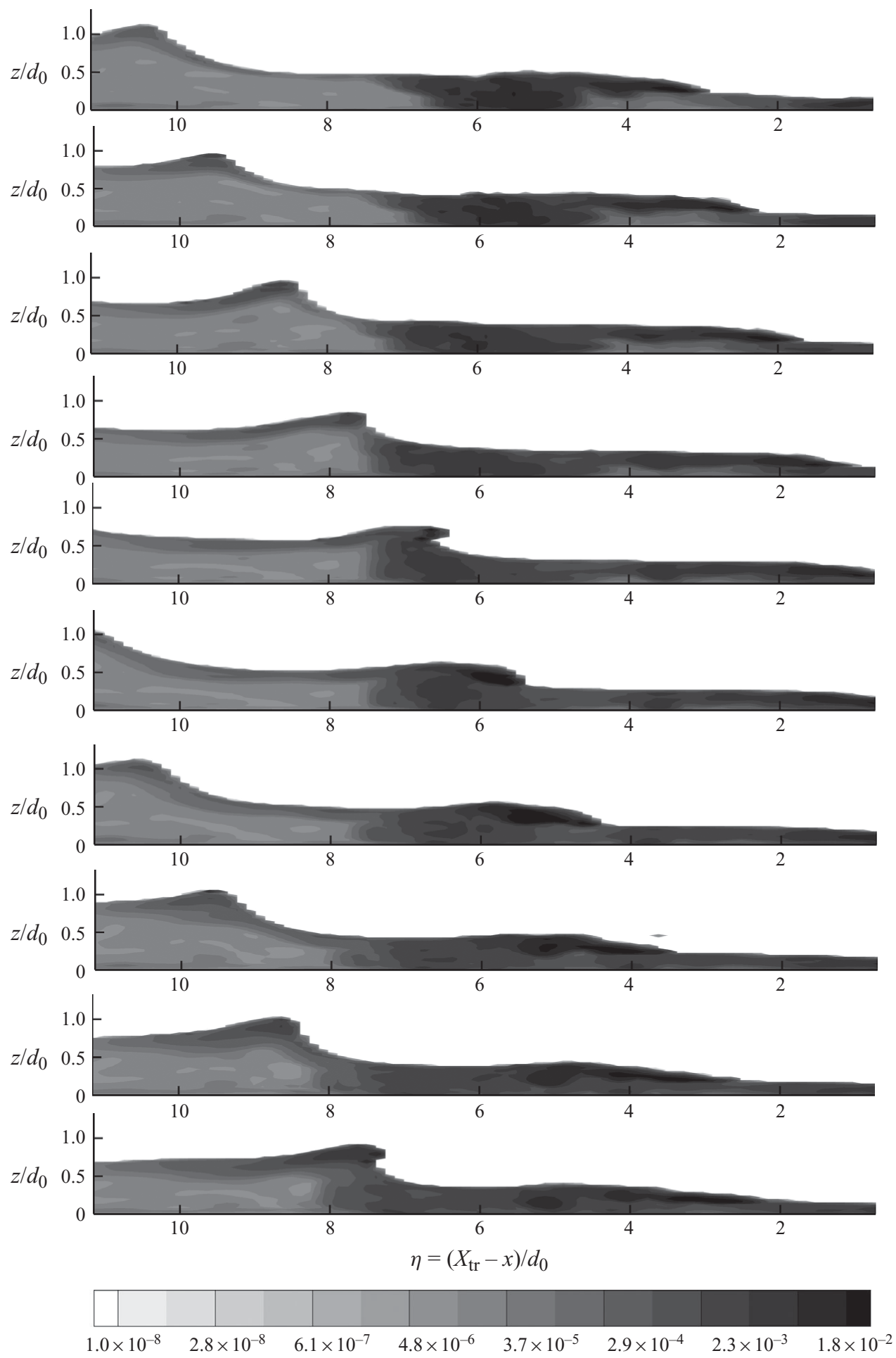

FIGURE 19. Spanwise-averaged TKE distributions in the liquid phase during wave breaking, from $t=3.4 \mathrm{~s}$ to $t=5.2 \mathrm{~s}$ at $0.2 \mathrm{~s}$ intervals. The spanwise extent shown in the plots is $0.77 \mathrm{~m} \geqslant x \geqslant 4.12 \mathrm{~m}$. 
(a)

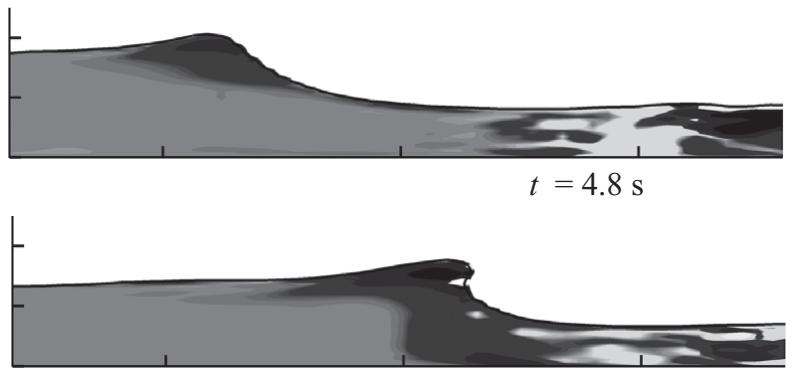

$1.0 \times 10^{-2}$

$7.8 \times 10^{-4}$

$3.3 \times 10^{-4}$

$-1.1 \times 10^{-4}$

$t=5.2 \mathrm{~s}$

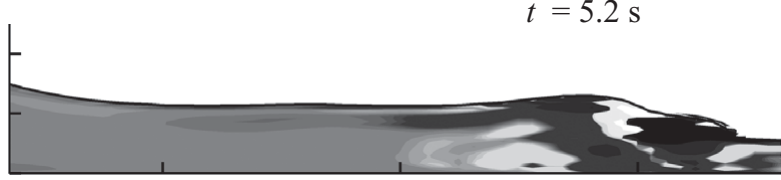

$-5.5 \times 10^{-4}$

$-1.0 \times 10^{-3}$

$-1.0 \times 10^{-1}$

(b)

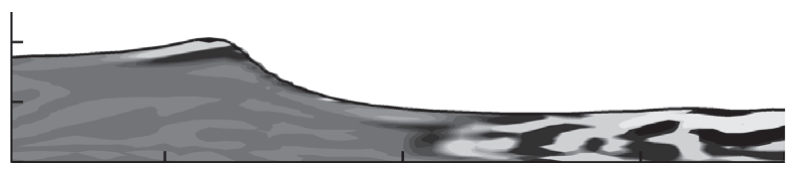

$t=4.8 \mathrm{~s}$

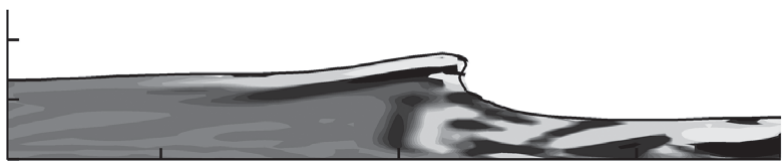

$t=5.2 \mathrm{~s}$

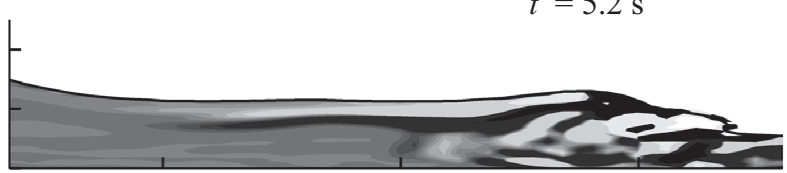

$t=5.6 \mathrm{~s}$

(c)

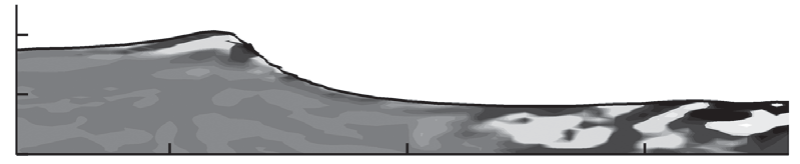

$t=4.8 \mathrm{~s}$

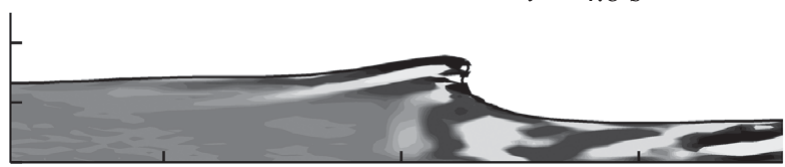

$1.0 \times 10^{-2}$

$7.8 \times 10^{-4}$

$3.3 \times 10^{-4}$

$-1.1 \times 10^{-4}$

$-5.5 \times 10^{-4}$

$t=5.2 \mathrm{~s}$

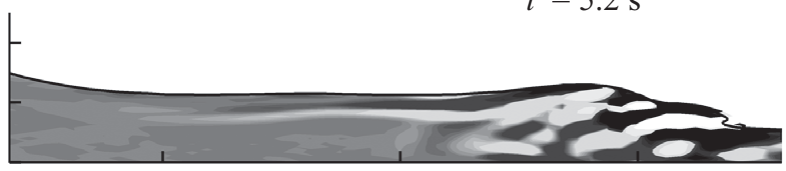

$-1.0 \times 10^{-3}$

$-1.0 \times 10^{-1}$

$$
t=5.6 \mathrm{~s}
$$

FIGURE 20. Spanwise-averaged distributions of $(a)$ TKE production, $(b)$ turbulent transport and $(d)$ pressure diffusion during the breaking event, from $t=4.8 \mathrm{~s}$ to $t=5.6 \mathrm{~s}$ at $0.4 \mathrm{~s}$ intervals. The spanwise extent shown in the plots is $0.72 \mathrm{~m} \geqslant x \geqslant 2.81 \mathrm{~m}$. 
of the turbulent processes, the zones of interest are the inner and outer surf zones. Positive exchange - or production - is already sustained at the crest of waves as they approach inner surf zone 3, and in the impact area. This shear-induced mechanism is understood as energy transfer from the $2 \mathrm{D}$ wave to the $3 \mathrm{D}$ fluctuating field. Negative exchange - or energy backscatter - from the fluctuating field to the mean and the waves is seen to take place in the inner zone as well as at the edge of breaking zone 2, in between plunger impacts. Turbulence in the inner zone is in fact well organized even if it seems to feature random exchange of energy between the various modes. At the edge of the breaking zone, negative production or backscatter coincides with local reversal of the flow behind the wave. Further frames beyond those depicted in figure 20 show that the zone of negative turbulent production spans the seaward side of the post-impact aftermath from the surface to the seabed.

Turbulent transport and pressure diffusion distributions shown in figure $20(b, c)$ support the conclusion that turbulence is strongly active and sustainable in the surf zone. Furthermore, the plots point to the subtle process of turbulence diffusion or spreading down - almost across flow - to the water core flow evoked in Svendsen (1987) and Christensen et al. (2002). The downward spreading of energy originates from the thin surface sublayer alongshore which forms quasi-instantaneously at the wave birth. In fact, this thin zone of positive pressure diffusion and turbulent transport is associated with maximum vertical TKE gradient $(\partial K / \partial z)$ discussed previously, and may thus be termed the inward surface sublayer. The upper thin layer squeezed between the inward surface sublayer and the free surface, now featuring negative pressure diffusion and turbulent transport, corresponds to $\partial K / \partial z<0$. Turbulence in the surf zones propagates seawards by the mean flow with the shoreward advancement of the wave crest.

The above analysis clearly demonstrates that solving for both phases either side using accurate interface tracking schemes has the major advantage of providing a clear picture of what might be expected to happen in the thin surface sublayers, a feature that obviously remains out of reach of any other computational technique.

\section{Global energy budget}

The previous sections have quantified turbulence in a relatively local manner in the wave-breaking flow, whether at points in the $(x, z)$ plane of the dominant 2D flow or as averages in zones featuring distinct flow patterns. Comparison between zones as well as comparison with the energy of the dominant $2 \mathrm{D}$ wave flow motivates consideration of the global energy budget. In a flow with no net inflows or outflows, the energy budget reduced to

$$
E_{p}+E_{k}+T K E=\epsilon_{0}-\int_{0}^{t} \epsilon \mathrm{d} t .
$$

Additional terms would be required in the case of net inflow or outflow. The terms in (10.1) are computed here in the following manner:

$$
\begin{aligned}
\text { potential energy: } E_{p} & =\int_{V} \rho g z \mathrm{~d} V-E_{p}^{q u i e s c e n t}, \\
\text { kinetic energy: } E_{k} & =\frac{1}{2} \int_{V} \rho\left\langle u_{i}\right\rangle^{2} \mathrm{~d} V, \\
\text { turbulent kinetic energy: } T K E & =\frac{1}{2} \int_{V} \rho\left\langle u_{i}^{\prime}\right\rangle^{2} \mathrm{~d} V,
\end{aligned}
$$



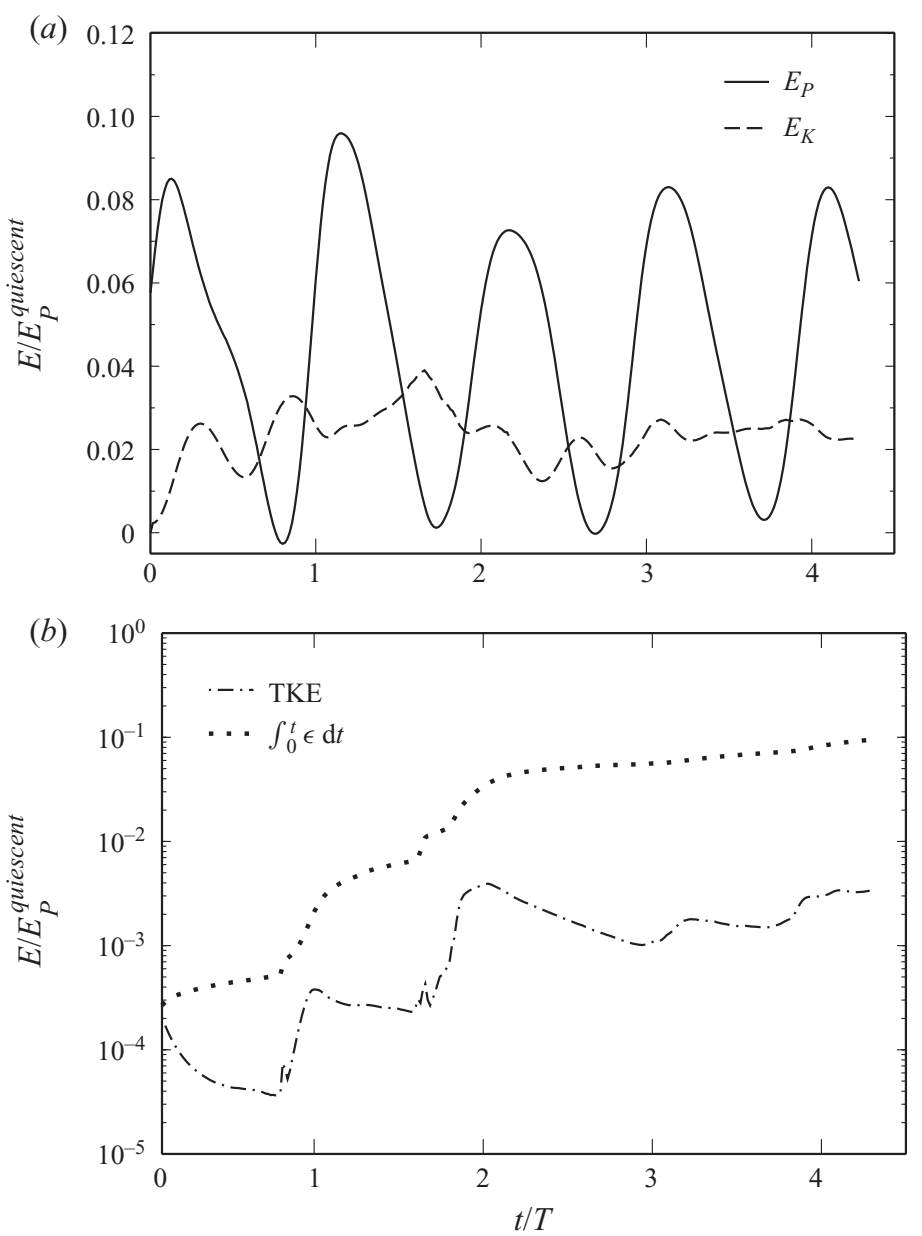

Figure 21. $(a, b)$ Time evolution of energy components in the global budget:- - , potential energy; --, kinetic energy; --.-, TKE; $\cdots$, dissipation.

$$
\text { dissipation: } \int_{0}^{t} \epsilon \mathrm{d} t=\sum_{t=0}^{t_{N}}\left(\mu+\mu_{T}\right) \int_{V}\left(\frac{\mathrm{d}\left\langle u_{i}\right\rangle}{\mathrm{d} x_{j}}\right)^{2} \mathrm{~d} V .
$$

Using $z$ for the height above the seabed in physical (unrotated) coordinate space, $z=0$ denotes the seabed level at $x=0$ in the rotated coordinate space (at which $d=0.321$ ). The constant $E_{p}^{q u i e s c e n t}$ zeroes the potential energy based on the quiescent flat free surface.

In accordance with the theory, the potential energy is periodic with a well-defined cycle in terms of wavelength and amplitude. For the most part, troughs in the mean kinetic energy signal depicted in figure 21 coincide with peaks in the potential energy signal and vice versa. While the potential energy is periodic, the kinetic energy signal is quasi-periodic, augmented by additional local extrema brought in by the wave dynamics. The action of the wave generator at the offshore boundary is most intense in the potential energy signal. While the potential and kinetic energies associated with $2 \mathrm{D}$ wave motion tend to dominate, dissipation is seen to match maximum potential energy levels only in a later stage. The most rapid rise in dissipation coincides with the 




Figure 22. (Colour online) Snapshots of coherent structure (CS) distributions for $t=6.0 \mathrm{~s}$, based on $(a)$ the liberal $-\lambda_{2}=5$ criterion in both liquid and gas phases and $(b)$ stricter $-\lambda_{2}=50$ criterion.

most intense interface wrinkling and turbulence activity generated by wave plunging. Before the first impact, turbulence (in the strictly defined sense of fluctuations over the spanwise-averaged field) is almost negligible during the start-up period $(T>1.4)$.

\section{Coherent structures}

Having identified key flow zones of intense turbulence production, destruction and transport, it is expected that coherent structures (CS) coincide with these areas. As such, the form of these structures is also a matter of interest here. The $-\lambda_{2}$ approach introduced by Jeong \& Hussain (1995) for coherent structures eduction is used in the current work. A first indication of the distribution of coherent structures throughout the flow is presented in figure 22 , for $t=6.0 \mathrm{~s}$. Use of a rather loose criterion $-\lambda_{2}=5$ is shown in the figure to result in high concentrations of CS, with many structures appearing large and not clearly separated from other structures. If the criterion is tightened to $-\lambda_{2}=50$, some structures disappear altogether, while the remaining structures become more clearly defined from adjacent ones. The higher $-\lambda_{2}$ criterion is effective in delineating genuinely strong vortical structures from weaker local velocity fluctuations. Further assessment of coherent structures is therefore based on the use of higher values of $-\lambda_{2}$. One general trend seen in figure 22 is that the water-sided structures are most concentrated shoreward of breaking, as well as nestled underneath the crest in the shoaling zone. The other obvious trend is that air-sided structures are more prevalent and persistent. 


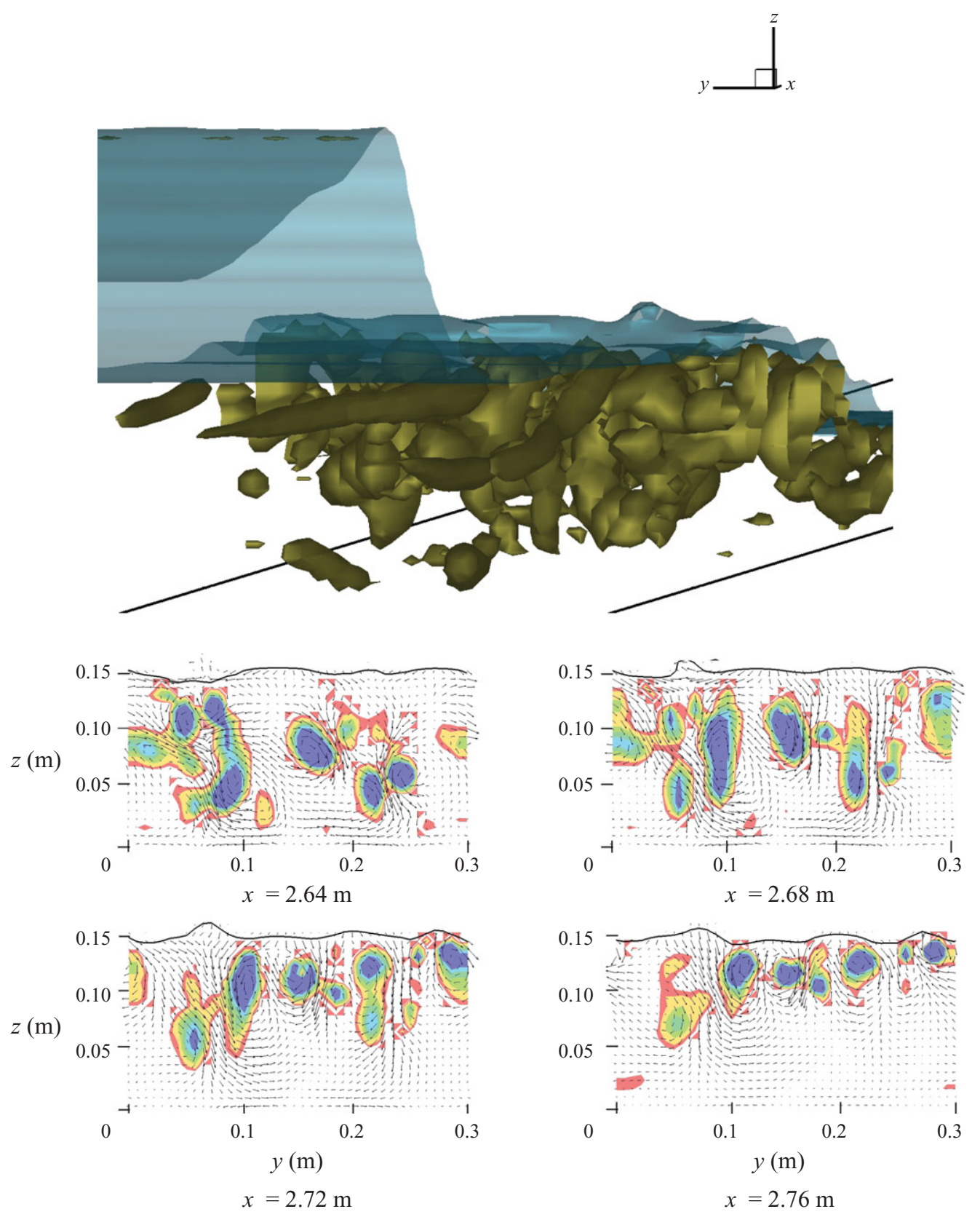

FIGURE 23. (Colour online) Obliquely descending eddies in the outer surf zone after the plunging event, at $t=6.0 \mathrm{~s}$ and using $-\lambda_{2}=20$. The flooded contours in the slices at constant $x$ are slices through the eddies highlighted in the top plot using the $-\lambda_{2}$ criterion.

For the purpose of identifying dominant types of coherent structures and mechanisms for their generation and dissipation, $-\lambda_{2}=20$ is chosen for the water flow. This value combines strictness criteria on positive CS identification with good preservation of physical detail; the result is shown in figure 23. While most of 
the water-side coherent structures are of quasi-streamwise type, figure 23 highlights occasional reorientation in the outer surf zone into obliquely descending eddies. Appearing in the plunging aftermath well after the plunger impact on the free surface, the obliquely descending eddies highlighted in figure 23 resemble in shape those reported by Nadaoka et al. (1989). Compared with the obliquely descending eddies in the CD paper, the eddies depicted in figure 23 are slightly shorter. The cross-sectional lower plots in the figure show the coherent structures beneath the surface to be rather well organized. Nadaoka et al. (1989) observed that the 2D structure of horizontal vortices in the water flow breaks down through the formation of vortices that tend to extend obliquely downwards. These were found to originate in the areas of maximum strain rate between the horizontal $2 \mathrm{D}$ vortices, and to be oriented roughly along the principal axis.

For coherent structures in the airflow, the highly stringent $-\lambda_{2}=50$ criterion shows (as in figure 24) the strongest CS to be formed subsequent to wave breaking. The airflow coherent structures are initially generated in the form of vortices of nearuniform 2D elliptical cross-section. With the major axis of the ellipse parallel to the interface, the elliptical cross-section of the vortex lies near-flat just above the level of the breaking wave. These initial 2D vortices of near-uniform profile in the longshore direction are then superseded by the creation of large obliquely ascending eddies, which also tend to persist in the airflow. The CS themselves also tend to undergo fragmentation, coalescence and reorientation. The net effect of these processes in the airflow is not only the occasional creation of other types of coherent structures such as hairpin vortices, but also the enduring presence of obliquely ascending vortices. In successive periods, a 2D vortex enters each time a new wave period (as identified by the peak in wave height to the left of the domain) begins, and is destroyed upon collision with the existing surf-zone airflow CS.

\section{Aeration and bubble entrapment}

Air entrainment resulting from plunger impacts on the water surface is depicted in figure 25, showing downward spread of the air-water foamy region. The result shown reflects air entrainment associated with one wave at various instances: $t=2.40 \mathrm{~s}$ and $t=2.50 \mathrm{~s}$. The $2 \mathrm{D}$ surface roller associated with wave plunging is the initial stage of air entrainment under the free surface. The $t=2.70 \mathrm{~s}$ frame shows major air entrainment to result from the impact of the wave jet ahead of the plunger. At instant $t=2.80 \mathrm{~s}$ and beyond, the entrained air seems to be entrapped: air pockets are dragged further under the free surface than induced by the momentum of the impacting jet. The entrained air that reaches essentially to the bottom of the seabed in the surf zone is significantly dispersed, as indicated by the void fractions decreasing with depth. This mechanism is essentially a mixing one that is very likely correlated to subsurface production, transport and diffusion of turbulence as discussed in the context of figure 20. Quantitatively, contour levels of void fractions subsequent to entrainment fall in the range $\alpha=0.2-0.3$, consistent with the results of Lamarre \& Melville (1991). Plots for later stages $(t=5.30 \mathrm{~s}$ and $t=5.60 \mathrm{~s})$ in figure 25 show that the aeration by the preceding plunging events does not persist. Shoreward transport prior to wave breaking re-compresses the aerated sea ahead of the plunging impact, which, together with the next breaking event, promotes disengagement of air from the surf zone. Disengagement of air entrained at preceding breaking events helps to maintain macroscopic balance of air volume fraction. In short, the entrainmententrapment-disengagement repeats with successive wave-breaking events. 
$t=1.9 \mathrm{~s}$

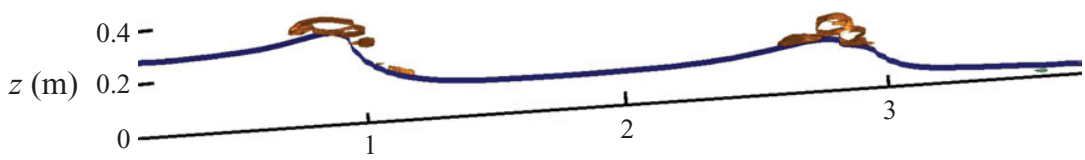

$t=2.4 \mathrm{~s}$

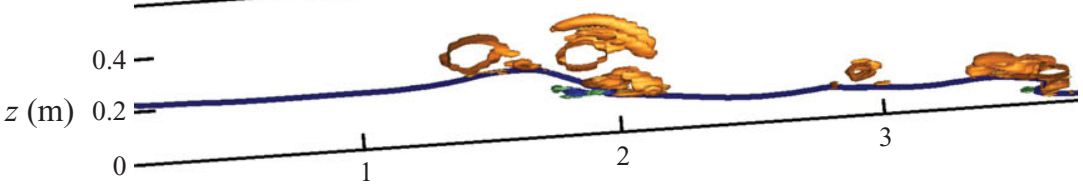

$t=2.9 \mathrm{~s}$

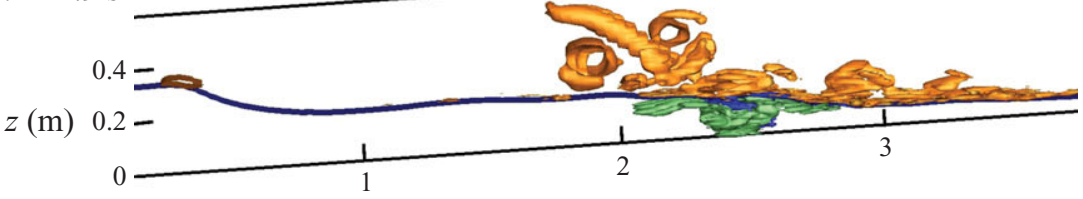

$t=3.6 \mathrm{~s}$

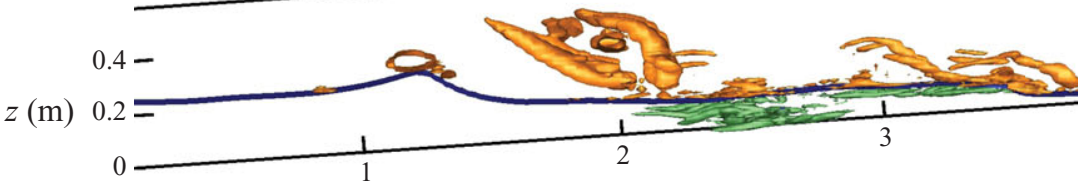

$t=5.0 \mathrm{~s}$

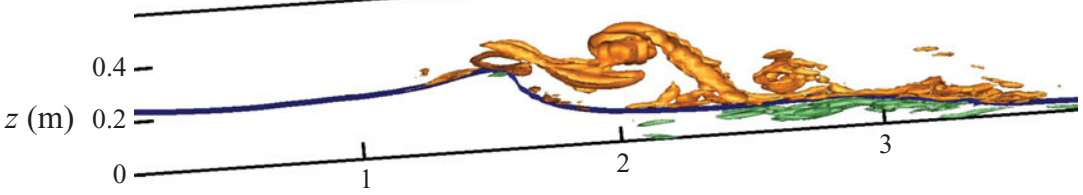

$t=6.0 \mathrm{~s}$

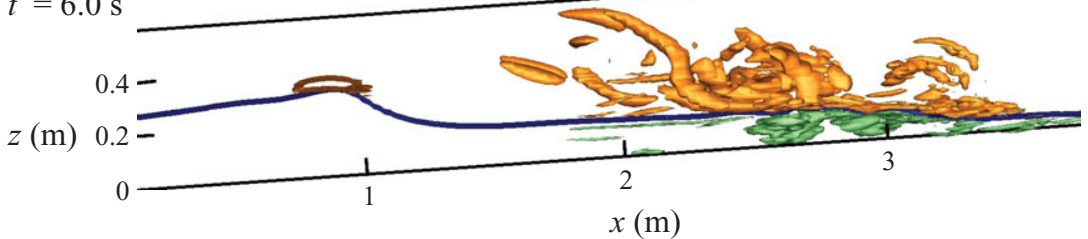

FIGURE 24. (Colour online) Initial creation of gas-sided CS by the wave breaking and the subsequent coalescence and reorientation of smaller-scale CS into larger-scale obliquely ascending vortices; $-\lambda_{2}=50$ in this case. 



FIGURE 25. Entrainment, entrapment and disengagement of small air bubbles in water due to wave breaking, as represented by contours of the void fraction in the geometric centreplane of the domain. 


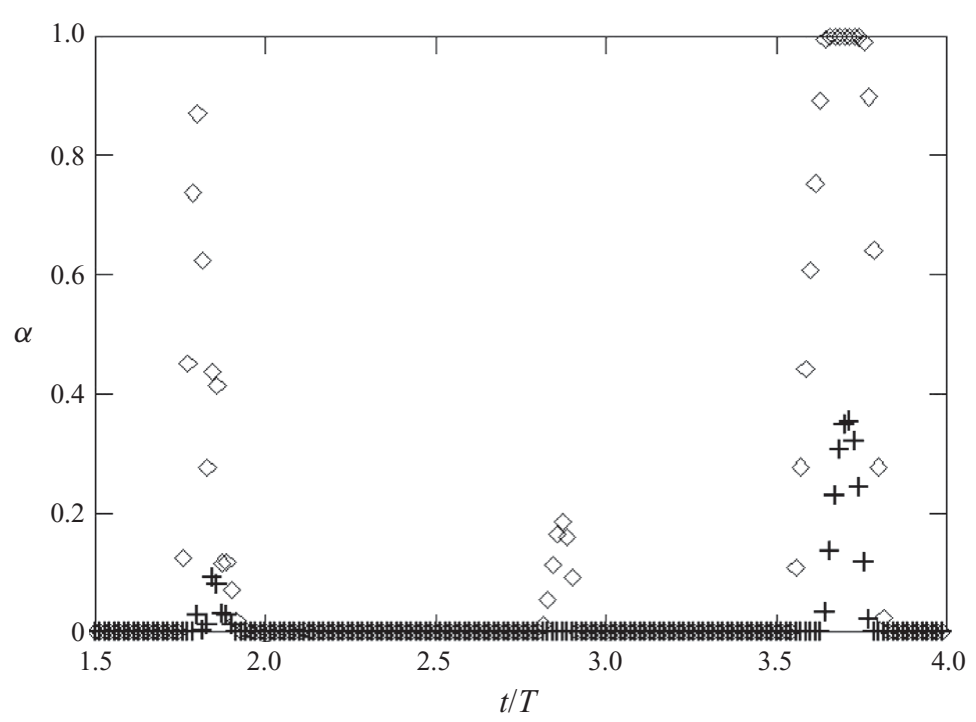

FIGURE 26. Time signal of void fraction at two points along the $x=2.3 \mathrm{~m}$ coordinate location in the domain.

To gain further insight into the transport of entrapped air by seaward and shoreward currents, void fraction time signals are recorded (figure 26). The signals are taken at two adjacent cell centres just below the quiescent free-surface level at $x=2.3 \mathrm{~m}$ and at $y$ centreplane. Smaller $\alpha$ signal never reaches $\alpha=1$, which may be indicative of entrained bubbles crossing the recording location. In contrast, for the second signal, it is difficult to ascertain whether the data reflect actually entrained bubbles or rather the passage of the free surface past the recording location. For both signals, the duration of air entrainment represents a small fraction of the total wave period $(10 \%)$, which is similar to the experiment of Cox \& Shin (2003). Applying a model of the form

$$
\frac{\alpha}{\alpha_{a v}}=a \frac{t}{T} \exp \left(-b \frac{t}{T}\right)
$$

is achievable but not particularly predictive, because figure 26 features increase and decay in void fraction at nearly the same rate. The difference in behaviour is possibly a result of limitations in the model represented by (12.1) in presuming $\alpha=0$ at $t / T=0$. In any case, the LEIS results presented in figure 26 are in line with the experimental observation. It is confirmed in particular that aeration does not persist till the arrival of the next breaker. The duration of the aeration at a point also does not persist longer than the $T / 8$ duration that Watanabe et al. (2005) indicated to be the time scale between the commencement of plunging and subsequent splash-up process. The observations from figure 26 suggest that a significant part of the near free-surface degassing occurs rapidly before the commencement of subsequent free-surface events, while the remaining entrapped air disengages later on due to recompression of the aerated sea extent.

\section{Energy spectra}

Energy spectra have been extracted using the spanwise-averaged velocity fluctuations at various points on a grid of $(x, z)$-coordinate locations. The 

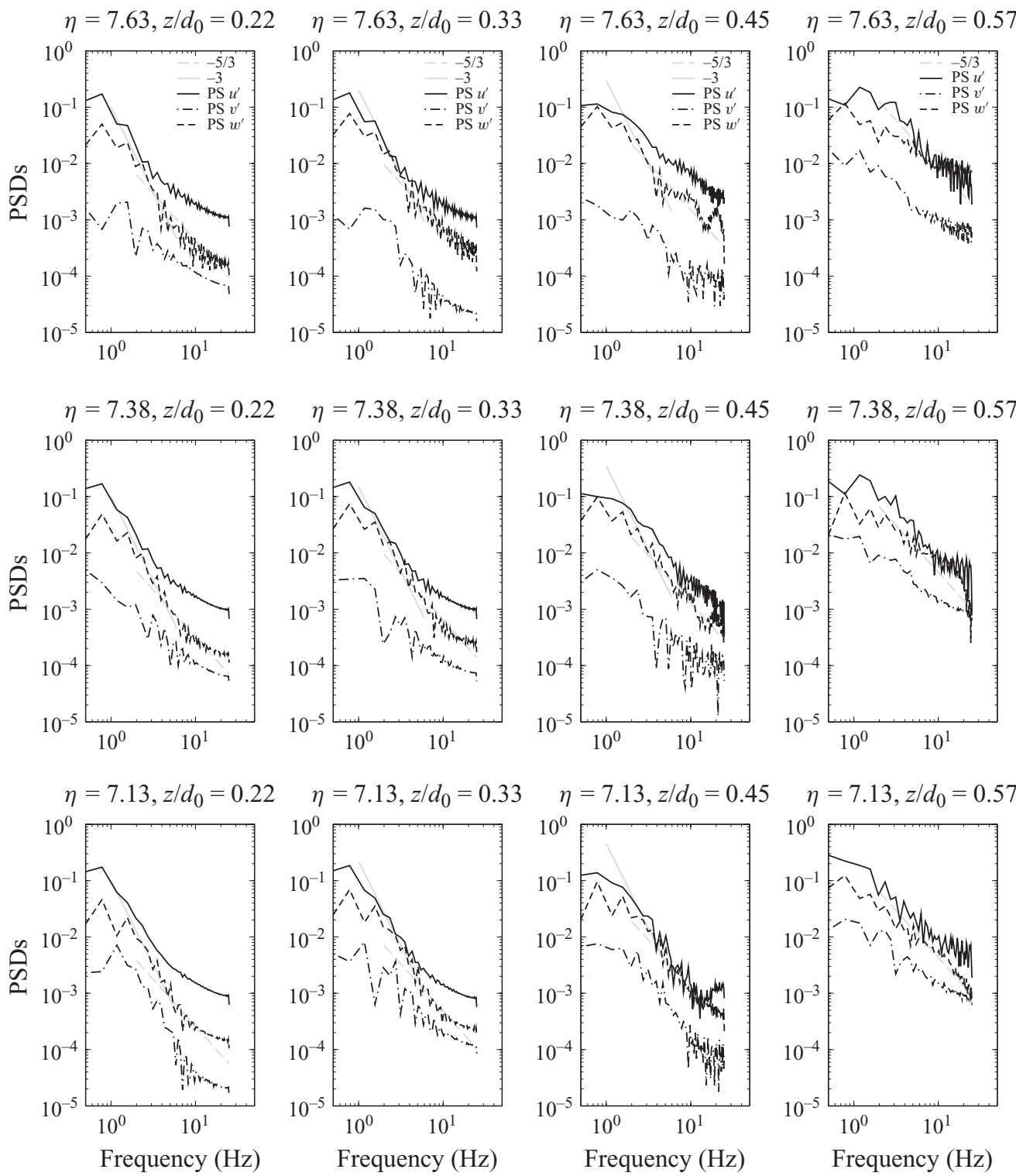

FIGURE 27. Energy spectra at various $(x, z)$-locations in the flow.

$x$-coordinates chosen span an area from just upstream of the breaking point to well downstream of the plunger impacts. For each $x$-coordinate, multiple $z$-coordinates are chosen such that points span from deep in the liquid phase to the gas phase. Energy spectra have been extracted from the time signals of all velocity components, using a sample size of 128 data points from the fully developed flow and a time spacing $\delta t=0.02 \mathrm{~s}$. Figure 27 presents the energy spectra at locations $x=1.90-2.06 \mathrm{~m}$ (7.6> $>>7.1$ ), spanning the region of the breaking point. Even in such a small streamwise subsection of the flow, the figure describes transitions into and away from 3D turbulence, with decay slopes being close to $2 \mathrm{D}$ turbulence $\left(k^{-3}\right)$, but sharper than $k^{-5 / 3}$. As the waves break, $k^{-3}$ shifts to $k^{-5 / 3}$ because two-dimensionality of the flow is now complemented by $3 \mathrm{D}$ turbulent small scales formed by breaking events, and 
energy is quickly dissipated following the cascade process. The transition from steeper slopes to the standard Kolmogorov K41 slope is a well-known feature of breaking waves, with the transition in energy spectrum slopes from -3 (2D turbulence) to $-5 / 3$ (fully developed 3D turbulence) noted by a number of authors (Lemmin et al. 1974; Battjes \& Sakai 1981; Hattori \& Aono 1985; Schlicke 2001) is predicted here, too. In Battjes \& Sakai (1981), the slopes were found to scale with $k^{-3}$ near the breaking point and with $-5 / 3$ further downstream, because the waves were spilling breakers. Results of the current study indeed show that the energy spectrum measurements of Battjes \& Sakai (1981) for spilling breakers are applicable to the case of weakplungers as well. For plungers, a -3 slope in the sea below the free surface at the breaking point is consistent with high TKE levels, coinciding with locally strong streamwise-oriented vortical structures that are not being transported seawards.

\section{Conclusion}

Large-eddy and interface simulation has been used to predict complex breaker events above a plane slope bed. The coupled air-liquid and surface displacement solutions were simultaneously obtained by the VOF interface tracking scheme. Turbulence was dealt with using LES with an SGS model accounting for turbulence decay at the surface. The model has first been validated for the spilling breaker problem of Ting \& Kirby $(1994,1996)$ in two and three dimensions, wherein the simulation results have been phase-averaged over 15 wave-breaking periods. The simulation of the main problem (proposed by Christensen \& Deigaard 2001) was intended to reproduce the scenario of a weak plunger, albeit involving substantially higher waves which permit development of more sustained turbulence. The ejection of a jet to impact the free surface ahead of the breaking wave and the dominant seaward transport of kinetic energy by the mean flow confirm that the wave-breaking simulated here is of plunging type. However, the lack of significant splash-up indicates that the waves are weak plungers, sharing some characteristics with spillers. Experimental correlations suggest that the increase in wave height adopted in this study (as compared with the $\mathrm{CD}$ weak plunger case) should have converted the wave from plungers to spillers.

The simulated flow is quite consistent in behaviour with the predicted theory and experiment, in which the initial processes leading to wave steepening are twodimensional, whereas the breaking is inherently three-dimensional. Wave growth and propagation are due to energy transfer from the mean flow to the wave modes, as well as transport of mean momentum by these modes. The transport of TKE can be seen to be shoreward along with the roller and seaward with the undertow observations consistent with those of Ting (2001). In accordance with Lamarre \& Melville (1991), air entrainment post-plunger impact has been found to be a highly dissipative process. The vigorous downwelling-upwelling pattern developing at the head and tail of each breaker is shown to enhance the streamwise and cross-depth variance and generates both negative and positive energy exchange (or production) terms in the water surface sublayer. The balance of production and dissipation in the TKE budget is altered by breaking waves. Turbulent transport, pressure diffusion and convective work are quite substantial sources and sinks in the budget, in particular in the inner and outer surf zones.

Small-scale breaking is seen to coincide with high levels of 3D turbulence in the surf zone, favouring the formation of streamwise and obliquely descending eddies. This is shown to be mostly generated during breaking events following jet impact and 
penetration through the free surface across depth. An analysis of void fraction profiles shows that breaking effectively increases free-surface 3D wrinkling by $O\left(10^{1}\right)-O\left(10^{2}\right)$. Prior to plunger impact, the generation of micro-breaking wrinkles (as compared with the $2 \mathrm{D}$ wrinkling of the organized wave motion) coincides with particularly intense 2D surface deformations. Two-dimensional surface deformations are intense enough to trigger small-scale breaking around the crest of the steepening wave and in the jet thrown forward during plunging. The higher density of coherent structures above the free surface coincides with the prevailing $-5 / 3$ power law for TKE decay. Transitions from a -3 power for a $2 \mathrm{D}$ turbulence decay to $\mathrm{a}-5 / 3$ power slope for $3 \mathrm{D}$ turbulence is obtained here too, consistent with most experimental studies dealing with wave breaking. Fully 3D decay slopes are persistent in the inner and outer surf zones in particular. The obliquely ascending nature of most of the airflow coherent structures, coupled with the form drag behaviour across the entire free surface, represents phenomena worth taking into account in formulating near-interface treatments for scalar transfer processes.

We thank Professor G. Yadigaroglu for his support to this work. The feedback and comments of Professor J. Magnaudet are also acknowledged. We also thank Professor J. Kirby for making his experimental data available to us. This work started while the authors were affiliated with the Institute of Energy Technology (IET), ETH Zürich. Since then, P.L. was supported by the CSIRO Energy Transformed Flagship. Computations were performed using the NCI National Facility in Canberra, Australia, which is supported by the Australian Commonwealth Government.

This paper is dedicated to the memory of my beloved mother, Zohra Romana Lakehal. That her memory remains present for ever, like the memory of sea waves (D.J.).

\section{REFERENCES}

Banner, M. L. \& Phillips, O. M. 1974 On the incipient breaking of small scale waves. J. Fluid Mech. 65, 647-656.

Battues, J. A. 1975 Surf similarity. In Proc. 14th Coastal Engrg. Conf., ASCE, New York, 466-480.

Battues, J. A. \& Sakai, T. 1981 Velocity field in a steady breaker. J. Fluid Mech. 111, 421-437.

Botto, L., Narayanan, C., Fulgosi, M. \& Lakehal, D. 2005 Effect of near-wall turbulence enhancement on the mechanisms of particle deposition. Intl J. Multiphase Flow 31, 940-956.

Brackbill, J. U., Kothe, D. B. \& ZemaCh, C. 1992 A continuum method for modeling surface tension. J. Comput. Phys. 100, 335-354.

Calhoun, R. J. \& Street, R. L. 2003 Turbulent flow over a wavy surface: neutral case. J. Geophys. Res. 106, 9277-9293.

Chang, K. A. \& LiU, P. L.-F. 1999 Experimental investigation of turbulence generated by breaking waves in water of intermediate depth. Phys. Fluids 11 (11), 3390-3400.

Christensen, E. D. 2006 Large eddy simulation of spilling and plunging breakers. Coast. Engng $\mathbf{5 3}$ (5-6), 463-485.

Christensen, E. D. \& Deigaard, R. 2001 Large eddy simulation of breaking waves. Coast. Engng 42, 53-86.

Christensen, E. D., Walstra, D. -J. \& Emerat, N. 2002 Vertical variation of the flow across the surf zone. Coast. Engng 45, 169-198.

Cox, D. T. \& Shin, S. 2003 Laboratory measurements of void fraction and turbulence in the bore region of surf zone waves. J. Engng Mech. 129, 1197-1205.

Duncan, J. H. 1996 Spilling breakers. Annu. Rev. Fluid. Mech. 33, 519-547. 
Fadlun, E. A., Verzicco, R., Orlandi, P. \& Mohd-Yusof, J. 2000 Combined immersed-boundary finite-difference methods for three-dimensional complex flow simulations. J. Comput. Phys. 161, 35-60.

Fenton, J. D. 1985 A fifth-order Stokes theory for steady waves. J. Waterway Port Coastal Ocean Engng 111, 216-234.

Fulgosi, M., Lakehal, D., Banerjee, S. \& De Angelis, V. 2003 Direct numerical simulation of turbulence in a sheared air-water flow with a deformable interface. J. Fluid Mech. 482, 319-345.

Galvin, C. J. 1968 Breaker-type classifications of three laboratory beaches. J. Geophys. Res. 73, 3651-3659.

Gemmrich, J. R. \& Farmer, D. M. 2004 Near-surface turbulence in the presence of breaking waves. J. Phys. Oceanogr. 34, 1067-1086.

GeurTs, B. J. 2002 Buoyant turbulent mixing in shear layers. In Advances in Turbulence IX - Proc. of the Ninth European Turbulence Conference (ed. I. P. Castro \& P. E. Hancock). CIMNE.

Goring, D. G. 1979 Tsunamis: the propagation of long waves onto a shelf. $\mathrm{PhD}$ dissertation, California Institute of Technology.

HatToRi, M. \& AONO, T. 1985 Experimental study on turbulence structures under breaking waves. Coast. Engng Japan 28, 97-116.

Jenkins, A. D. 2002 Do strong winds blow waves flat? Proc. WAVES 2001, in Ocean Wave Measurement and Analysis (B. L. Edge \& J. M. Hemsley), vol. 1, 494-500. American Society of Civil Engineers.

JeOng, J. \& Hussain, F. 1995 On the identification of a vortex. J. Fluid Mech. 285, 69-94.

Komori, S., Nagaosa, R., Murakami, Y., Chiba, S., Ishit, K. \& Kuwahara, K. $1993 b$ Direct numerical simulation of three-dimensional open-channel flow with zero-shear gas-liquid interface. Phys. Fluids A 5 (1), 115-125.

Labourasse, E., Lacanette, D., Toutant, A., Lubin, P., Vincent, S., Lebaigue, O., Caltagirone, J.P. \& Sagaut, P. 2007 Towards large eddy simulation of isothermal two-phase flows: governing equations and a priori tests. Intl J. Multiphase Flow 33, 1-39.

Lakehal, D., Milelli, M. \& Smith, B. L. 2002 Large-eddy simulation of bubbly turbulent shear flows. J. Turbul. 3, 1-21.

Lakehal, D., Reboux, S. \& Liovic, P. 2006 SGS modelling for the LES of interfacial gas-liquid flows. La Houille Blanche 6, 125-131.

LAM, K. \& BANERJEe, S. 1992 On the condition of streaks formation in a bounded turbulent flow. Phys. Fluids A 4 (2), 306-320.

Lamarre, E. \& Melville, W. K. 1991 Air entrainment and dissipation in breaking waves. Nature 351, 469-472.

Lemmin, U., ScotT, J. T. \& CZApski, U. H. 1974 The development from two-dimensional to three-dimensional turbulence generated by breaking waves. J. Geophys. Res. 19, 3442-3448.

Lin, C. \& Hwung, H. H. 1992 External and internal flow fields of plunging breakers. Exp. Phys. 12, 229-237.

Lin, M.-Y., Moeng, C.-H., Tsai, W.-T., Sullivan, P. P. \& Belcher, S. E. 2008 Direct numerical simulation of wind-wave generation processes. J. Fluid Mech. 616, 1-30.

Lin, P. \& LiU, P. L.-F. 1998 A numerical study of breaking waves in the surf zone. J. Fluid Mech. 359, 239-264.

Liovic, P. \& LAKeHAL, D. 2007 a Interface-turbulence interactions in large-scale bubbling processes. Intl J. Heat Fluid Flow 28, 127-144.

Liovic, P. \& LaKeHaL, D. $2007 b$ Multi-physics treatment in the vicinity of arbitrarily deformable gas-liquid interfaces. J. Comput Phys. 222, 504-535.

Liovic, P. \& LaKeHal, D. 2009 Interface-turbulence interactions and bubble dynamics. In Proc. 7th Intl Conf. CFD Mineral Process Industries, CSIRO, Melbourne, 1-6.

Liovic, P., Liow, J.-L. \& Rudman, M. 2001 A volume-of-fluid (VOF) method for the simulation of metallurgical flows. ISIJ Intl 41, 225-233.

Liovic, P., Rudman, M., Liow, J.-L., Lakehal, D. \& Kothe, D. B. 2006 A 3D unsplit-advection volume tracking algorithm with planarity-preserving interface reconstruction. Comput. Fluids 35, 1011-1032.

Lombardi, P., DeAngelis, V. \& Banerjee, S. 1996 Direct numerical simulation of near-interface turbulence in coupled gas-liquid flow. Phys. Fluids 8, 1643-1665. 
Lubin, P., Vincent, S., Abadie, S. \& Caltagirone, J. P. 2006 Three-dimensional large eddy simulation of air entrainment under plunging breaking waves. Coast. Engng 53 (8), 631-655.

Melville, W. K. 1996 The role of surface-wave breaking in air-sea interaction. Annu. Rev. Fluid. Mech. 28, 279-321.

Moum, J. N. \& Sмyтh, W. D. 2001 Upper ocean mixing. In Encyclopedia of Ocean Sciences, vol. 6, 3093-3100. Academic Press.

Nadaoka, K., Hino, M. \& Koyano, Y. 1989 Structure of the turbulent flow field under breaking waves in the surf zone. J. Fluid Mech. 204, 359-387.

Narayanan C. \& Lakehal, D. 2006 DNS of particle-laden mixing-layers. Part I. One-way coupled flow and dispersed-phase features. Phys. Fluids 18 (9), 093302.

Peregrine, D. H. 1983 Breaking waves on beaches. Annu. Rev. Fluid. Mech. 15, 149-178.

Reboux, S., Sagaut, P. \& Lakehal, D. 2006 Large-eddy simulation of sheared interfacial two-fluid flow using the variational multiscale approach. Phys. Fluids 18 (10), 105105.

Roelvink, J. A. \& Stive, M. J. F. 1989 Bar-generating corss-shore flows mechanisms on a beach. J. Geophys. Res. 94 (C4), 4785-4800.

SCHLICKE, T. 2001 Breaking waves and the dispersion of surface films. PhD thesis, University of Edinburgh.

Smith, E. \& Kraus, N. C. 1991 Laboratory study of wave-breaking over bars and artificial reefs. $J$. Waterway Port Coastal Ocean Engng 117, 307-325.

Sullivan, P. P. \& McWilliams, J. C. 2002 Turbulent flow over water waves in the presence of stratification. Phys. Fluids 14, 1182-1195.

Sullivan, P. P., McWilliams, J. C. \& Melville, W. K. 2004 The oceanic boundary layer driven by wave breaking with stochastic variability. Part 1. Direct numerical simulations. J. Fluid Mech. 507, 143-174.

Sullivan, P. P., McWilliams, J. C. \& Moeng, C. H. 2000 Simulation of turbulent flow over idealized water waves. J. Fluid Mech. 404, 47-85.

Svendsen, I. A. 1987 Analysis of surf zone turbulence. J. Geophys. Res. 92 (C5), 5115-5124.

SvendSEN, I. A. 2006 Introduction to Nearshore Hydrodynamics. Advanced Series on Ocean Engineering, vol. 24. World Scientific.

Thorpe, S. A. 1993 Energy loss by breaking waves. J. Phys. Oceanogr. 23, 2498-2502.

Thorpe, S. A. 1995 Dynamical processes at the sea surface. Prog. Oceanogr. 35, 315-352.

Thorpe, S. A., Osborn, T. R., Jackson, J. F. E., Hall, A. J. \& Lueck, R. G. 2003 Measurements of turbulence in the upper-ocean mixing layer using Autosub. J. Phys. Oceanogr. 33, 122-145.

TiNG, F. C. K. 2001 Laboratory study of wave and turbulence velocities in a broad-banded irregular wave surf zone. Coast. Engng 43, 183-208.

Ting, F. C. K. \& Kirby, J. T. 1994 Observations of undertow and turbulence in a laboratory surf zone. Coast. Engng 24, 51-80.

Ting, F. C. K. \& KirbY, J. T. 1995 Dynamics of surf-zone turbulence in a strong plunging breaker. Coast. Engng 24, 177-204.

Ting, F. C. K. \& Kirby, J. T. 1996 Dynamics of surf-zone turbulence in a spilling breaker. Coast. Engng 27, 131-160.

Tsai, C.-P, Chen, H.-B., Hwung, H.-H., \& Huang, M.-J. 2005 Examination of empirical formulas for wave shoaling and breaking on steep slopes. Ocean Engng 32, 469-483.

Watanabe, Y. \& SaEki, H. 1999 Three-dimensional large eddy simulation of breaking waves. Coast. Engng 41, 281-301.

Watanabe, Y., Saeki, H. \& Hosking, R. J. 2005 Three-dimensional vortex structures under breaking waves. J. Fluid Mech. 545, 291-328.

Zhao, Q., Armfield, S. \& Tanimoto, K. 2004 Numerical simulation of breaking waves by a multi-scale turbulence model. Coast. Engng 51, 53-80. 